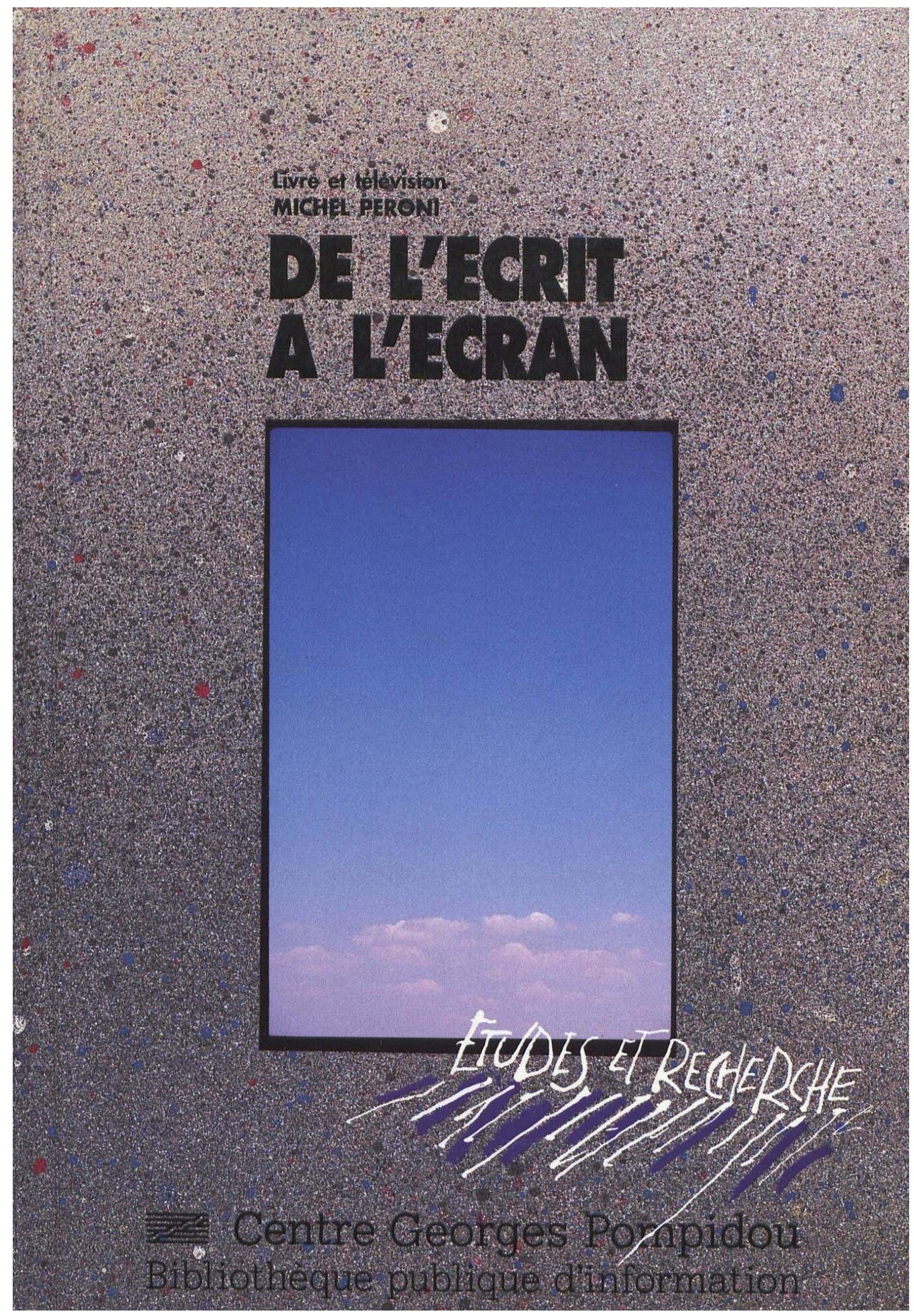




\section{De l'écrit à l'écran}

\section{Michel Peroni}

DOI : 10.4000/books.bibpompidou. 1720

Éditeur : Éditions de la Bibliothèque publique d'information Année d'édition : 1991

Date de mise en ligne : 5 juin 2015

Collection : Études et recherche

ISBN électronique : 9782842461751

\section{Sbooks}

http://books.openedition.org

\section{Édition imprimée}

ISBN : 9782902706334

Nombre de pages : 214

\section{Référence électronique}

PERONI, Michel. De l'écrit à l'écran. Nouvelle édition [en ligne]. Paris : Éditions de la Bibliothèque publique d'information, 1991 (généré le 02 février 2021). Disponible sur Internet : <http:// books.openedition.org/bibpompidou/1720>. ISBN : 9782842461751. DOI : https://doi.org/10.4000/ books.bibpompidou. 1720 .

Ce document a été généré automatiquement le 2 février 2021. II est issu d'une numérisation par reconnaissance optique de caractères.

(C) Éditions de la Bibliothèque publique d'information, 1991

Conditions d'utilisation:

http://www.openedition.org/6540 
Livre et télévision, voilà une question d'actualité. Les émissions littéraires font-elles lire davantage? Ou au contraire dispensent-elles de lire?

Une chose est sûre, de Lectures pour tous, la plus ancienne, à Apostrophes, la plus célèbre, les émissions littéraires ont réussi au fil des années à faire entrer le livre sur la scène télévisuelle, puis à élargir leur public aux « bons » lecteurs.

Le livre à la lucarne, soit! Mais s'agit-il encore du même livre, celui qu'un lecteur solitaire ouvre, lit, et referme quelques heures plus tard, sa lecture terminée? Pas exactement. Les émissions littéraires participent d'une socialisation du livre qui - espace public et timing obligent - tend à en exclure la lecture. Le livre sur un plateau, c'est le livre sans la lecture, ou la lecture figurée.

Et l'auteur? L'invitation du livre à l'a télévision ne requiert pas l'existence préalable d'un écrivain ; elle institue plutôt un auteur comme devant assumer la responsabilité du livre devant la scène publique. Ce n'est alors plus l'écriture mais bien la personne de l'auteur qui donne au livre sa valeur. L'auteur en scène, ou de l'écrivain à l'homme de parole.

C'est ce que vous trouverez dans ce livre, enrichi de nombreux morceaux choisis de vos émissions littéraires préférées. Et maintenant... place à la lecture !

\section{MICHEL PERONI}

Michel Peroni est sociologue au CRESAL (unité associée au CNRS, à Saint-Etienne). Il est l'auteur dans cette même collection («Études et recherches », éditions BPI/Centre Pompidou) de Histoires de lire, lecture et parcours biographique (1988). 


\section{SOMMAIRE}

\section{Introduction}

Chapitre 1. Entre lecture et télévision : la culture en question

Le livre et la television : une question d'actualité

La lecture et la télévision : la question de la concurrence

Les émissions littéraires et la lecture : la question de l'effet induit

Les émissions littéraires : des émissions (presque) comme les autres

\section{Chapitre 2. Le livre sur un plateau : la lecture figurée}

Le livre à la télévision : une question d'actualité

Le livre dans les émissions littéraires

Le livre sans la lecture

Le livre à tout bout de champ

Chapitre 3. L'auteur en scène : de l'écrivain à l'" homme de parole»"

Quand l'auteur paraît

L'author's studio

L'auteur soi-même

Pour être écrivain, on n'en est pas moins homme

\section{Conclusion}

Annexes 


\section{Introduction}

1 «Le livre et la télévision ${ }^{1}$ », voilà une question d'actualité, ou plutôt une question dont l'actualité est récurrente comme en témoigne cette série de grands titres: "Les émissions littéraires amènent-elles le spectateur à lire davantage ${ }^{2}$ ?", "Les émissions littéraires à la TV ne font pas lire ${ }^{3}$ ", "La télévision ne fait plus lire ${ }^{4}$, " La télé vole au secours du livre ${ }^{5} » . .$. Simple donnée d'expérience, cette énumération, pour incomplète qu'elle soit, n'en constitue pas moins une prémisse obligée de la recherche. Deux remarques suffisent à situer toute l'importance d'une réelle prise en compte d'une problématisation sociale qui préexiste comme telle à notre propre questionnement.

2 D'abord, l'ampleur et la constance de la couverture médiatique sont une indication implicite de la nature de ce qui est ici en cause. En clair : si la question des rapports de la lecture et de la télévision peut de la sorte faire périodiquement la «une» de l'actualité, si elle ne concerne pas seulement les professionnels du livre, c'est que ne se réduisant pas à la question d'une simple concurrence entre pratiques, elle a bel et bien la culture elle-même pour enjeu. On est loin ici de la concurrence couramment dénoncée entre la fréquentation des stades ou des salles de cinéma et la transmission télévisée des rencontres sportives ou des films.

3 Ensuite, d'un point de vue méthodologique, la thématisation dont fait « naturellement » l'objet la question des rapports entre lecture et télévision ne saurait être une ressource, par exemple, une réserve d'informations objectives, pour le questionnement sociologique. Quand bien même elle se présente le plus souvent sous une forme quasi sociologique, avec notamment un recours systématique aux données statistiques. Tout au contraire, une telle thématisation fait partie intégrante de la question elle-même. En effet, de par la force d'imposition attachée aux formes de son traitement social, le domaine du questionnable se trouve ici ramené à un simple arbitrage entre deux formations discursives stabilisées: un discours téléphile - la télévision encourage la lecture - contre un discours téléphobe - la télévision dissuade la lecture.

4 Ces deux remarques préliminaires permettent d'établir les conditions de pertinence de la réflexion qui s'engage, ainsi que les types d'investigations requis pour les assurer. Il s'agira dans un premier temps de considérer les figures stéréotypées du discours sur le rapport entre livre et télévision dans leur factualité même (c'est-à-dire du point de vue 
de l'efficience attachée à leur existence en tant que fait social). L'analyse visera, dans cette perspective, à répondre à plusieurs interrogations.

Comment les reformulations successives de la relation entre livre et télévision, au gré des transformations du média, d'une problématique de la concurrence à une problématique de la complémentarité des pratiques lectorales et télévisuelles, s'inscrivent dans un cadre commun caractérisé par certains traits distinctifs. Soit la séparation radicale entre livre et télévision, tous deux objets de pratiques distinctes. Puis, la prise en compte de la pratique de la télévision du point de vue exclusif de son effet sur la pratique du livre. Enfin, l'évaluation par inférence des effets de la télévision sur la lecture, cette dernière étant l'horizon référentiel véritable de la problématisation ordinaire du rapport livre-télévision.

Comment ces problématiques, aussi contradictoires soient-elles, s'entretiennent et se légitiment également dans une prétendue saisie objective et protocolisée de la réalité des pratiques, études d'audience d'un côté, nombre de livres lus de l'autre. Comment, quelle que soit la problématique, la problématisation constante du rapport livretélévision a pour efficacité pratique d'affirmer et réaffirmer implicitement la valeur qui est attachée au livre. Comment en d'autres termes elle opère une véritable construction sociale du livre comme objet culturel par excellence.

7 La présente recherche, si l'on n'y prend garde, pourrait fort bien elle-même, au pire, n'être qu'une reformulation actualisée des mêmes problématiques (eu égard, comme mentionné plus haut, aux transformations récentes du média), et, au mieux, venir arbitrer « scientifiquement » le conflit qu'elles entretiennent sur le sens de la causalité (en procédant à l'examen systématique de la conformité méthodologique des différents protocoles de production des données).

8 Tout en prenant appui sur les données accumulées par le Service d'études d'opinion (SEO) puis le Centre d'études d'opinion (CEO) et aujourd'hui Médiamétrie, auxquelles il convient d'ajouter les données ponctuelles issues d'enquêtes réalisées par les chaînes de télévision ou la presse, cette première série d'investigations ne sera pas l'étude quantitative attendue. Loin d'être en effet cet instrument technique, toujours perfectible, par lequel approcher toujours plus près une réalité des pratiques qui serait notre objet substantiel, les « données » statistiques seront par elles-mêmes, notre objet véritable. C'est que leur production et leurs commentaires contribuent d'une manière décisive (puisque sous couvert de saisie objective) à faire passer dans l'ordre des faits, les prétendus effets de la télévision sur la lecture; et à objectiver par là un modèle culturel déterminé : celui de la culture savante.

9 Au terme de ce premier chapitre, et alors seulement, il nous sera possible de nous détourner des formes de problématisation ordinaires du rapport livre-télévision. Ce qui impliquera de remettre en cause la séparation entre les pratiques du livre et de la télévision, ainsi que la saisie des secondes du strict point de vue de leurs effets sur les premières. Mais, cela ne saurait suffire. Car, en dernière analyse, il s'agit d'échapper aux apories de l'inférence qui menacent, bien au-delà d'ailleurs du problème ici posé, toute entreprise de mesure des pratiques culturelles. Il est tout aussi improbable d'évaluer l'effet de la télévision sur la lecture, que de rapporter par exemple la lecture au nombre de livres achetés ou possédés. Inférer en l'occurrence un accroissement ou une diminution du niveau de la lecture, non directement mesuré quant à lui, d'une plus ou moins grande consommation d'émissions littéraires, risque fort de n'engendrer que des reformulations des mêmes propositions indéfiniment réversibles. Vouloir échapper 
aux apories de l'inférence semble dans ces conditions devoir requérir l'exclusion de la lecture du domaine d'investigation. Se refuser pourtant à une telle exclusion implique de manière autrement plus radicale d'abandonner le terrain des pratiques. C'est un véritable recadrage du questionnement qui est alors opéré, désignant la présence du livre à la télévision comme objet directement appréhendable, en lieu et place de cet objet lointain qu'était le niveau de lecture. Recadrage qui implique le passage d'une approche quantitative à une approche plus qualitative, quasi ethnographique qui interrogera en diachronie la place ménagée au livre à la télévision et en synchronie, les formes que prend aujourd'hui cette présence.

10 A l'argument méthodologique qui a jusque-là suffi à justifier cette redéfinition de l'objet que l'on vient d'accomplir, le moment est venu d'associer un argument théorique susceptible de rendre raison à la volonté déjà manifestée de ne pas exclure la lecture du domaine de la recherche. Or, un tel argument renvoie à une position critique développée ailleurs ${ }^{6}$ par rapport au champ de cette sociologie bien improprement dite «sociologie de la lecture ».

11 Pour une sociologie qui se donne le plus souvent pour objet l'étude des pratiques d'usage du livre (de l'imprimé), socialement différenciées et objectivement repérables (modes d'information et d'acquisition des livres, systèmes de classification et de rangement des livres dans les intérieurs, temps consacré au livre, genres possédés...), la lecture proprement dite, lorsqu'elle n'est pas tout simpement évacuée, est au plus inférée des variables déterminantes que sont le niveau d'instruction et l'origine sociale.

12 En d'autres termes, la lecture n'est paradoxalement pas l'objet privilégié par cette « sociologie de la lecture » que l'on pourrait plus sûrement qualifier de « sociologie du livre» (comme production culturelle). Dans le cadre de la problématique de la domination culturelle qui est la sienne, "on suppose "qu'assimiler" signifie nécessairement "devenir semblable à" ce qu'on absorbe et non "le rendre semblable" à ce qu'on est, le faire sien, se l'approprier ou réapproprier ${ }^{7}$ ». Il n'en reste pas moins que, comme le souligne R. Chartier, « de mêmes textes et de mêmes livres sont l'objet de déchiffrements multiples, socialement contrastés; ce qui doit nécessairement amener à compléter l'étude statistique des distributions inégales par celle des usages et des emplois. Ajouter donc à la connaissance des présences du livre celle des façons de lire ${ }^{8} . .$. » et, ajouterons-nous, de ses significations.

13 La validité et la pertinence de cette recherche tiennent donc à la possibilité de concilier la nécessité méthodologique de centrer l'approche sur le livre et l'intérêt théorique du développement d'une sociologie compréhensive de la lecture. Précisément, la redéfinition de l'objet résultant de notre orientation méthodologique risque fort d'inscrire une fois de plus la recherche dans le cadre d'une sociologie des usages sociaux, médiatiques en l'occurrence, du livre. Aussi, faisons nous le pari qu'une analyse des rapports entre le livre et la télévision peut effectivement s'inscrire dans le cadre d'une réflexion sur la signification de la lecture et les attributions de sens dont elle est l'objet. Et ceci, de deux manières distinctes correspondant chacune aux deuxième et troisième temps de nos investigations. D'une part, les conditions même de la présence du livre à la télévision ainsi que les différents types de dispositifs mis en place pour l'assurer rendent figurable à chaque fois un type de représentation de la lecture. Cependant, la scène médiatique n'est pas seulement un espace de figuration de représentations existantes; c'est également le lieu d'émergence et de diffusion de nouveaux systèmes de signification. 
14 D'autre part donc, de par la force d'imposition caractéristique du média, les formes inédites de la présence télévisée du livre, qui sont autant de réponses pratiques à des impératifs proprement télévisuels, constituent par elles-mêmes un opérateur des plus efficaces de la redéfinition dont la lecture fait actuellement l'objet.

Que le lecteur non informé et sur le point de s'engager dans la première partie de cet ouvrage consacrée aux données statistiques ne regrette pas de ne pas être plutôt en train de regarder la télévision où passe peut-être en ce moment même quelque émission sur les livres qu'il affectionne... C'est l'affaire de quelques instants: les parties suivantes lui permettront de retrouver le petit écran dans le livre. Mais auparavant, la lecture exige - n'est-ce pas? - un effort, un tout petit effort, sans commune mesure finalement avec les satisfactions qu'elle est susceptible d'apporter.

\section{NOTES}

1. C'est le titre d'un dossier du Nouvel Observateur, mars 1982.

2. Le Monde, 30 novembre 1966.

3. Le Figaro, 13 mai 1975.

4. Le Matin, 21 avril 1983.

5. Le Figaro, supplément télévision, 20 novembre 1987.

6. M. Péroni, «Lecture et parcours biographique », in Pour une sociologie de la lecture, éd. du Cercle de la librairie, 1988.

7. Certeau Michel de, L'Invention du quotidien, tome 1, Les Arts de faire, UGE, 1980.

8. Chartier Roger, «Du livre au lire », Pratiques de la lecture, sous la dir. de R. Chartier, Rivages, 1985. 


\title{
Chapitre 1. Entre lecture et télévision : la culture en question
}

\author{
«Certaines sortes de communication sur \\ certaines sortes de sujets, portées à la \\ connaissance de certaines personnes, dans \\ certaines sortes de conditions, ont certaines \\ sortes d'effets. » (Berelson)
}

\section{Le livre et la television : une question d'actualité}

1 «En vingt ans, la France a basculé dans l'ère de la télévision : un million de récepteurs en 1959, quinze millions aujourd'hui. La lecture pourrait-elle résister, les mots tenir bon devant les images? Pour le savoir, L'Express a fait interroger par l'institut LouisHarris un échantillon de 2000 personnes représentatif de la population française âgée de 18 ans et plus. Conclusion sans appel, surprise, stupeur même: le pourcentage des Français qui lisent a augmenté de $15 \%$.» La surprise est à la mesure du titre qui fait la couverture de ce numéro de L'Express du 4 novembre 1978 : « La France se met à lire »! Il est vrai que les chiffres manifestent une évolution sensible.

2 «A la question: "En dehors des journaux, magazines, revues, vous est-il arrivé de lire un livre au cours des trois derniers mois ?", 57 \% des Français âgés de 20 ans et plus répondent oui, $43 \%$ non. A une question comparable, en 1960, lors d'un sondage effectué par le Syndicat national des éditeurs, $42 \%$ avaient répondu oui, $58 \%$ non. Les chiffres avaient fait scandale, à l'époque. En vingt ans, la proportion a basculé, dans le bon sens. »

Que l'on ne se méprenne cependant pas sur notre propos : il ne s'agit pas pour nous de placer cette étude du rapport livre-télévision sous le signe de la stupeur provoquée par des chiffres aussi «satisfaisants ». Ce que nous voudrions au contraire problématiser à travers les différents sondages et autres données statistiques rassemblés dans cette première partie, et par delà leurs propres résultats, c'est la question commune dont ils procèdent. «Quels sont les effets de la télévision sur la lecture ?» Au-delà de l'examen méthodologique qui interroge la validité des réponses apportées: les données statistiques dont nous disposons sont-elles fiables? Au-delà de l'examen 
épistémologique qui considère la pertinence des réponses au regard de la question posée: les données statistiques dont nous disposons constituent-elles vraiment des réponses? C'est à l'examen critique de l'évidence de la question elle-même que nous entendons nous livrer ici ${ }^{1}$ : quel type de présupposé autorise donc à (ne) concevoir le rapport lecture-télévision (que) comme un rapport de causalité univoque; et plus encore, à ne concevoir télévision et lecture que du point de vue de leur rapport (problématique) ? Ainsi entendue, ce type d'analyse des statistiques sur la lecture et la télévision se doit de considérer, plus encore que les chiffres qu'elles produisent, leurs présupposés et commentaires; quand bien même et à plus forte raison encore, lorsque les chiffres, comme l'on dit, "parlent d'eux-mêmes ».

4 Tel est justement le cas du présent sondage de L'Express; et c'est précisément pour avoir fait en son temps la « une » de l'actualité qu'il peut plus que tout autre nous révéler le cadre implicite du questionnement générique sur le rapport entre télévision et lecture.

5 Tout d'abord, c'est le nombre de livres lus, quels qu'ils soient et à l'exclusion de tout autre type d'imprimé, qui tient lieu de critère d'appréciation de l'évolution de la lecture. Se féliciter, dans ces conditions, que cette dernière se fasse " dans le bon sens " revient à tenir la lecture pour une valeur en soi, sous l'argument implicite que la lecture c'est la culture. Dans une telle identité métonymique, il faut voir l'effet d'un double jugement prescriptif : d'un côté, la lecture est la condition de possibilité même de la culture (encore faut-il savoir lire, disposer d'une compétence au déchiffrage), de l'autre, elle en est l'accomplissement par excellence (encore faut-il aimer lire, être disposé à la lecture). Comme pratique indissociable d'une valeur, la lecture transcende la différenciation sociale. Sorte d'universel concret elle est aussi bien pratique distinctive de la culture lettrée qu'instrument de lutte contre l'illettrisme. "Satisfaisants ", les résultats du présent sondage le sont alors à double titre. D'un côté, comme l'indique le titre de l'article repris en couverture du numéro: «La France se met à lire », ce qui équivaut littéralement à «La France sort de l'illettrisme »; toutes formulations fondées sur l'idée d'une compétition (sur le modèle de la statistique électorale) sanctionnée par le fait majoritaire : la lecture dépasse la barrière des $50 \%$ !

De l'autre côté, l'évolution se fait " dans le bon sens ", dessinant ainsi implicitement un parcours lectoral univoque conçu comme un continuum non lecteur-gros lecteur. Quelle est en effet la carte de la lecture en France? « Deux noyaux durs et stables, un tiers d'étrangers au livre, $22 \%$ de passionnés. Entre les deux, une moitié de Français vient doucement mais sûrement à la lecture. Là se trouvent les nouveaux bataillons gagnés en vingt ans » (apprécions au passage la belle métaphore guerrière). La stabilité du noyau des gros lecteurs atteste ici que l'évolution se fait bien au détriment des nonlecteurs en même temps qu'elle en fixe le terme logique ; les faibles et moyens lecteurs $\mathrm{y}$ viendront tout aussi « doucement et sûrement » qu'ils sont venus à la lecture.

7 Mais prendre acte d'une telle évolution de la lecture, alors même qu'elle suscite l'enthousiasme, ne suffit pas, et le commentaire journalistique d'inscrire d'emblée les résultats du sondage dans un contexte spécifique : celui d'une "montée inéluctable de l'audiovisuel » supposée repousser la lecture « dans des retranchements désespérés ». A l'intérêt statistique de l'information s'ajoute ici un intérêt proprement dramaturgique : voilà que la lecture résiste... à la concurrence, mieux encore, au devenir hégémonique de la télévision! Ce n'est que dans le cadre de cet antagonisme que les résultats du présent sondage prennent véritablement tout leur sens, toute leur portée. Ils peuvent être l'objet de deux types de commentaires renvoyant chacun à une interprétation de la 
nature de cet antagonisme : la télévision s'opposant dans un cas à la lecture entendue comme pratique (participant du domaine des loisirs), dans l'autre à la lecture entendue comme valeur (participant du domaine de la culture). Mais vérifions à l'instant comment le passage d'une interprétation à l'autre s'opère insensiblement.

8 En premier lieu, répétons-le, il convient de se féliciter d'une évolution de la lecture qui va «dans le bon sens »; la satisfaction est ici à la mesure de la surprise. C'est que le présent sondage confirme par ailleurs que la télévision se pose aujourd'hui en concurrent direct du livre dans le domaine des loisirs même si le faible écart en pourcentage entre les deux pratiques ( $73 \%$ pour la télévision, $69 \%$ pour la lecture) tient pour partie à l'hétérogénéité de leur critère de mesure respectif (dans un cas on comptabilise ceux qui regardent la télévision "tous les jours ou presque ", et dans l'autre, ceux qui "ont lu des livres les douze derniers mois»). D'ailleurs d'autres sondages portant sur le temps consacré en moyenne à ces deux pratiques, et donc sur leur intensité respective, accusent pour leur part cet écart ${ }^{2}$ (télévision 1 heure 44, lecture 27 minutes par jour).

9 Retenons simplement que les chiffres, quels qu'ils soient, ne font ici que confirmer cette idée observable par quiconque, d'une concurrence entre les pratiques. Le temps de loisir n'étant pas extensible, regarder la télévision revient à amputer le temps virtuellement disponible pour la lecture. Dans cette formulation élémentaire du rapport concurrentiel livre-télévision, si la télévision (concurrente de la lecture dans l'utilisation du temps de loisir) se pose en obstacle de fait à la culture, simple mass média, elle ne remet pas directement en cause les prérogatives culturelles de la lecture. C'est d'ailleurs précisément dans cette différence de nature entre les deux pratiques que peut se résoudre ce qui apparaît désormais comme un paradoxe : les Français n'ont pourtant jamais été aussi nombreux à lire ; et les jeunes tout particulièrement qui ont cependant grandi avec la télévision et dont on ne cesse d'affirmer qu'elle les a détournés de la lecture ${ }^{3}$.

10 A bien des égards, en effet, on peut considérer la télévision comme étant le contraire du livre: elle impose des horaires, une audience collective; tandis qu'il invite à une aventure individuelle. "Cette différence de statut entre le livre et la télévision a contenu la poussée de l'image et renforcé le livre... Mais, ne concluons pas trop vite à la victoire de l'écrit.

11 Notre sondage montre que la situation n'est pas stabilisée. En ce premier âge de la télévision, celui de l'image collective, le livre a réussi à se développer comme l'outil de la culture personnelle... Mais sa résistance reste fragile parce qu'elle repose sur les faiblesses - passagères - de la télévision ". Qu'en sera-t-il demain lorsque, grâce au progrès technique, son caractère collectif va disparaître ? Grâce à la transmission par câble, chacun pourra choisir son programme comme on choisit un livre; avec le magnétoscope, chacun pourra revoir comme on peut relire, en puisant dans sa «bibliothèque » personnelle d'images. En bref, « entre l'imprimerie et l'électronique, le sort de la culture est toujours en balance ». Voilà, on ne saurait être plus explicite, la question du rapport concurrentiel livre-télévision est finalement reformulée d'un point de vue prospectif : la télévision menace virtuellement la lecture sur le terrain même de la culture, préfigurant alors la transformation d'une culture lettrée en une culture de l'image.

12 Les analyses des transformations récentes de la télévision confirment quant à elles cette virtualité. Ainsi, pour P. Chambat et A. Ehrenberg4, la télévision échapperait 
aujourd'hui « au modèle de la consommation de masse centré sur le ménage et travaillé par les valeurs du confort, du standing et de la possession " au profit d'un "modèle de communication centré sur l'échange interindividuel et travaillé par les valeurs de l'activité et de la relation ». Or la transformation de l'imaginaire consommatoire qui régit nos rapports avec la télévision est précisément interprétée par ces auteurs en termes d'émergence d'une « culture de l'écran ».

Ils rappellent tout d'abord que les premières années de la télévision en France « ont été marquées par une rhétorique de type pédagogique intrinsèquement liée aux préoccupations de service public... L'image était destinée à frapper l'esprit des gens qui maîtrisent mal l'écrit... La télévision (média nouveau perçu comme plus efficace que l'écrit grâce à l'image et à son pouvoir de pénétration bien supérieur à l'école) était le moyen d'accès à la culture pour ceux qui en ont été exclus. »

$\mathrm{Au}$ cours des années soixante, c'est au contraire dans le cadre d'un modèle de consommation de masse centré sur le ménage et la famille que se diffuse la télévision. "La télévision est alors le plus souvent élaborée dans un modèle culturel que l'on peut appeler le "prêt à consommer"... [elle] se met à symboliser le loisir populaire sans effort et la détente familiale à domicile. L'imaginaire de la télévision la situe aux antipodes du temps contraint, à l'opposé des valeurs de l'effort et du recueillement que nécessite la ("vraie") culture. » Les critiques qui lui sont dès lors adressées stigmatisent à la fois la "médiocrité des émissions populaires ne demandant aucun effort personnel " et la " passivité de l'écoute liée à la nature du média ». Au nivellement de la programmation répond l'inertie d'un public fasciné.

Aujourd'hui, avec la surabondance d'images issue des nouvelles technologies de communication, cette thématique de la fascination est en voie de disqualification. "On croyait le téléspectateur halluciné par le petit écran, on découvre aujourd'hui qu'il se fait volage... Les moralistes d'hier cherchaient à arracher les gens à la télévision, les experts d'aujourd'hui ont pour principale préoccupation de les fidéliser. A un usage flottant, distrait, à éclipse de la télévision répond une stratégie de réimplication du téléspectateur dont l'interactivité est le mot d'ordre. » Parallèlement, la multiplication et la diversification des "objets à écran" semblent annoncer l'émergence d'une "culture de l'écran s'articulant, se substituant partiellement ou réaménageant la culture de l'imprimé ». L'écran devenant alors, comme ce fut le cas pour l'imprimé, support universel.

Ces éléments de réflexion sont un utile appui en ce qu'ils invitent à considérer l'opposition entre culture livresque et inculture télévisée, hégémonie pratique de la télévision et légitimité culturelle de la lecture, et même culture de l'imprimé et culture de l'écran, comme des schémas discursifs associés chacun à un moment de l'histoire du média. Dans ces conditions, nous ne saurions reprendre à notre compte de telles oppositions dans l'analyse des enquêtes et autres sondages périodiquement renouvelés sur le rapport qu'entretiennent la lecture et la télévision. Ce qui reviendrait à considérer les données statistiques comme l'indicateur par excellence susceptible de déterminer rien moins que le sort de la culture. Il s'agira, au contraire, de montrer dans notre analyse comment ces dits instruments de saisie objective de la réalité et de ses transformations sont eux-mêmes le produit de ces schèmes, que l'on peut dès lors proprement qualifier de cognitifs et dont ils contribuent en retour à renforcer l'évidence. 


\section{La lecture et la télévision : la question de la concurrence}

17 «Quel rôle joue donc la télévision par rapport à la lecture ? Fait-elle qu'on lit moins? Qu'on lit plus ? Qu'on lit mieux ? Ou tout simplement peut-elle faire qu'on lise ; c'est-àdire peut-elle amener au livre ces millions de lecteurs potentiels?» Telles sont les questions posées déjà, en juillet 1968, par une note de synthèse : «La télévision et la lecture ", exhumée des archives du ministère de la Culture et de la Communication. Rassemblant les données alors disponibles sur ces différents points ${ }^{5}$, cette note constitue un document exemplaire dans sa tentative de les organiser dans le cadre d'une problématique de la concurrence entre la télévision qui se diffuse alors et la lecture. Aussi, reprendrons-nous ici les grandes lignes de son argumentation avec les têtes de chapitre d'origine.

\section{L'hypothèse de la concurrence}

18 Où il apparaît que la pratique du livre est érigée en norme culturelle a priori par l'intermédiaire de l'argument de la concurrence télévisuelle.

19 Plusieurs enquêtes, datant de la fin des années 1950, sur le temps consacré à la lecture avant et après l'acquisition d'un téléviseur, donnent des résultats convergents. Pour ne reprendre que les chiffres de l'enquête ORTF de 1959: $47 \%$ des personnes interrogées disent lire autant ; $46 \%$ moins et $7 \%$ plus, qu'avant l'acquisition d'un téléviseur.

20 "Il semble donc, d'après ces pourcentages, que la télévision ait une influence plutôt défavorable sur l'activité de lecture, en tant que loisir. » D'autant qu'une enquête menée par les services de l'ORTF à Paris et en province, en février et mars 1961, révèle qu'avant l'acquisition du poste de télévision la distraction favorite des téléspectateurs était la lecture. Il est d'ailleurs significatif que $20 \%$ des téléspectateurs se retournent vers la lecture quand le programme de télévision ne leur plaît pas.

21 "A première vue, il semble donc que la télévision remplace pour un certain nombre de gens la lecture comme activité culturelle» (sic!). A cela, plusieurs raisons. Une raison d'ordre économique tout d'abord : « Le poste de télévision s'achète souvent à crédit ; les traites mensuelles grèvent lourdement et pendant de nombreux mois, le budget-loisir du foyer. Il est compréhensible que les autres postes de ce budget en pâtissent. ».

22 Le manque de temps est aussi une des explications les plus fréquemment données pour expliquer la non-lecture. Ainsi à la question "Quelles sont les raisons qui vous empêchent de lire davantage?", posée lors d'une enquête sur la lecture à SaintÉtienne $^{6}$, quels que soient l'âge et la catégorie professionnelle, le manque de temps vient en tête des réponses. Mais le manque de temps est-il ici simple justification ou raison véritable? «Le problème n'est sans doute pas si simple. Même si le manque de temps n'explique pas tout, la lecture est associée à l'idée d'effort ${ }^{7}$ et ceux qui ne disposent que de peu de temps de loisirs l'occupent plus volontiers à ce qu'ils considèrent comme étant davantage "détente". Les résultats obtenus par R. Kaes ${ }^{8}$ dans son étude sur les ouvriers et la culture en font foi.»

Enfin, les habitudes familiales peuvent accentuer cette tendance. En effet, « le moment privilégié pour la lecture reste le soir, après le travail (pour $73 \%$ des personnes interrogées par l'Ires). C'est également le moment où l'on se retrouve en famille. Or la 
lecture est par définition une activité de loisir individualisée. Par contre, le fait de regarder la télévision peut être une activité collective qui permet la discussion commune, fût-elle limitée, et qui donne l'impression de ne pas s'isoler, de "rester en famille". La structure familiale permet aussi à la télévision de s'enraciner facilement, comme type de loisir culturel vécu sur le mode collectif, et donc de supplanter la lecture."

On ne peut manquer ici de souligner l'adhésion tacite aux thèses formulées par R. Hoggart dans La Culture du pauvre et alimentées par la prise en compte de certains paradoxes dont celui-ci, tellement exemplaire qu'il est repris par J.-C. Passeron dans sa présentation de l'ouvrage au public français. «Si l'achat d'un poste de télévision est plus logique que celui d'une machine à coudre c'est que, dans les classes populaires, la préférence va toujours, quelle que soit l'exiguïté du budget, aux biens dont l'utilisation collective peut servir de support au rassemblement ou à la communion hé-donique de la communauté familiale, le repli sur la privauté ou même la promiscuité du foyer constituant le seul recours contre une condition qui serait autrement invivable ${ }^{9}$. " Aussi faudrait-il voir derrière l'hypothèse de la concurrence des pratiques, une théorie implicite de la culture populaire.

De fait, tandis que les données de base initiales concernaient « les téléspectateurs » en général, les explications invoquées convoquent ensuite une série de déterminants distinctifs pour leur part des conditions d'existence des couches populaires (contraintes de budget, de temps, habitudes familiales). Est ainsi produite la fiction d'un remplacement chez elles de la lecture par la télévision, comme activité culturelle... alors même que si la télévision a pu s'y diffuser massivement, c'est par sa prétendue intégration à une culture (i.e. " du pauvre ») préexistante, précisément antinomique par construction, de la lecture! Notons, en effet, que parmi les téléspectateurs déclarant lire ou acheter autant qu'avant l'acquisition de leur téléviseur figurent implicitement ceux qui ne lisaient pas ou n'achetaient pas de livre. L'invisibilité de l'inégalité d'accès originelle à la culture lettrée constitue donc la condition même de la problématisation du rapport lecture-télévision, en termes concurrentiels ${ }^{10}$.

\section{La télévision : « un guide pour la lecture », voire « une incitation à la lecture »}

Où il apparaît que la télévision ne peut accéder au rang de pratique culturelle légitime que si elle est au service du livre.

\section{Guider}

«Si les chiffres d'ensemble traduisent une diminution du phénomène global de lecture chez les téléspectateurs, ne serait-ce pas aussi en raison d'une sélection plus rigoureuse des livres qu'on achète et qu'on lit? C'est là d'abord une conséquence normale de certaines émissions comme Lecture pour tous dont la fonction manifeste est de guider le choix des téléspectateurs. » C'est en tout cas ce que semble confirmer l'étude sur SaintÉtienne, puisque $21 \%$ des interviewés (non-téléspectateurs compris) déclarent se servir de la télévision pour sélectionner leurs lectures. Et, quel que soit l'âge ou la catégorie socio-professionnelle, c'est presque toujours entre 20 et $25 \%$ que se situe ce 
pourcentage ${ }^{11}$. Par ailleurs, il apparaît que l'influence globale de la télévision opère une sélection des genres.

L'enquête de l'Ires fournit à ce sujet des éléments intéressants, (cf. tableau A en annexes). Elle montre que chez les concurrents du livre, les magazines subissent beaucoup plus que les quotidiens les effets de l'entrée de la télévision dans les foyers. En ce qui concerne les livres, qu'il s'agisse des romans ou des livres documentaires, il y a presque exactement le même nombre de gens à ne pas avoir changé leurs habitudes. Mais s'il n'y a que $4 \%$ de téléspectateurs à lire davantage de romans, il y en a $14 \%$ à lire plus de livres documentaires. "C'est surtout aux dépens du roman que semble donc s'être faite la sélection. »

29 Une enquête britannique, réalisée en 1957 et citée par Henri Dieuzeide, va d'ailleurs dans le même sens. Elle indique que « l'introduction de la télévision dans une ville fait généralement baisser de moitié la lecture des romans et quadrupler la lecture des ouvrages géographiques, des récits de voyage, des manuels techniques et des livres d'art. En un mot, la télévision paraît combler les aspirations romanesques en même temps qu'elle suscite une curiosité nouvelle pour la réalité12 $»$.

30 En guise de conclusion sur ce point, les auteurs de la note s'en remettent à l'autorité de Joffre Dumazedier. «Si on lit moins, peut-être lit-on mieux, parce que la télévision joue le rôle d'un guide du lecteur.» Se fondant sur les seules données que nous avons précédemment reproduites, on peut mesurer ce qu'une telle conclusion doit au parti pris axiologique.

\section{Inciter}

31 Mais ce n'est pas tout: "la télévision peut-elle faire mieux que d'aider à orienter le choix de ceux des téléspectateurs qui sont déjà des lecteurs ? Peut-elle vraiment jouer un rôle d'incitation à la lecture? " Incontestablement, répondent les auteurs, la télévision dispose pour ce faire de nombreux atouts.

32 Alors qu'un obstacle majeur à la lecture tient dans l'effort qu'elle nécessite, la télévision n'est quant à elle accompagnée d'aucune représentation de l'idée d'effort : « elle peut très bien familiariser les téléspectateurs avec les livres sans qu'une telle idée y soit associée. »

33 En outre, la télévision est supposée avoir les possibilités techniques de rendre simples et attrayants les problèmes les plus complexes, supprimant ainsi une partie de l'effort qui tient non à la lecture elle-même mais au sujet abordé. « Il peut s'en suivre un désir d'en savoir davantage qui amènerait au livre. »

34 «La télévision peut aider à combattre l'idée que les livres sont faits par des intellectuels pour des intellectuels. » Les interviews télévisées mais aussi des émissions du type portrait d'auteur peuvent contribuer à leur démythification.

35 La télévision peut faire en sorte que le livre cesse d'être perçu comme une chose à part, détachée de la vie réelle. «Elle peut aider à réintégrer le livre dans l'actualité, dans les préoccupations quotidiennes, dans la vie réelle que connaissent les téléspectateurs. »

36 Enfin, par le biais des émissions littéraires proprement dites, elle peut amener les livres aux téléspectateurs au lieu d'attendre que ceux-ci aillent aux livres. 
Or, de 1958 à 1963, puis 1967, la part d'audience de Lectures pour tous n'a cessé de se réduire (il faut ici tenir compte de l'introduction de la deuxième chaîne) passant de $50 \%$ à $30 \%$ et $5 \%$ et l'indice de satisfaction de $80 \%$ à $65 \%$ et $53 \%$.

En conséquence, s'agissant plus précisément des milieux populaires ici explicitement visés, les auteurs doivent bien reconnaître qu'«il n'y a pas d'incitation directe à la lecture qui passerait par le canal d'émissions spécialisées, type Lectures pour tous ». Ils retiennent cependant la possibilité que certaines émissions puissent provoquer chez les téléspectateurs le désir d'en savoir davantage sur quelque sujet que ce soit et l'amener ainsi à la lecture. "Peut-il y avoir incitation directe? Sans qu'il soit nécessaire de raisonner en termes de pourcentage, il semble cependant que l'on puisse répondre affirmativement. » La préférence même pour l'achat de livres documentaires « ne serait pas uniquement le fait de téléspectateurs opérant ainsi un simple transfert à l'intérieur de leur lecture, mais également de nouveaux lecteurs qui viendraient aussi à la lecture par souci de documentation sur un problème précis ».

De même que l'on présupposait des téléspectateurs d'aujourd'hui qu'ils étaient les lecteurs d'hier, on suppose cette fois une efficacité culturelle de la télévision comme incitation à la lecture. Plus qu'elles n'apportent une confirmation sur l'un ou l'autre de ces points, les enquêtes disponibles témoignent de la prégnance d'un modèle culturel associant la culture au livre, et ne reconnaissant par définition de positi-vité à la télévision qu'au service (public) de la lecture.

\section{Les émissions littéraires et la lecture : la question de l'effet induit}

40 Au regard de l'évolution de la télévision, la question cruciale, il y a vingt ans, de la concurrence des pratiques n'a plus de sens aujourd'hui. « En 1967, en effet, 57 \% des ménages étaient équipés d'un téléviseur et $51 \%$ des individus, qu'ils appartiennent ou non à un foyer équipé, regardaient la télévision "tous les jours ou presque". Vingt ans plus tard, les taux sont respectivement de $94 \%$ et $82 \%$. On mesure mieux ce qu'a de massif cette diffusion quand on se penche sur les durées d'écoute : chaque jour où il regarde la télévision, un adulte urbain y consacre 2,10 heures en moyenne, durée qui a augmenté de 20 minutes entre 1975 et $1986^{13}$. »

Dans le cadre d'une telle hégémonie pratique de la télévision, ce qui importe n'est plus tant de savoir si les gens regardent ou pas la télévision, mais ce qu'ils y regardent. A l'unité de la télévision, antagoniste de la lecture, fait place la différenciation des émissions, à laquelle correspond d'ailleurs une reconnaissance de la diversité des genres constitutifs de la lecture. Par elle-même, la télévision peut être désormais opérateur de distinction. Elle n'est plus, en effet, tenue pour étrangère à la culture, comme en témoigne dès 1973 l'enquête sur les Pratiques culturelles des Français. Non seulement, il y est consacré une partie à la télévision, mais est prise en compte dans la partie réservée à la lecture, l'écoute des émissions sur la littérature et les écrivains, au même titre que la lecture de revues spécialisées. Rompant avec la problématique de la concurrence, les enquêtes cherchent alors à appréhender « les effets réciproques des divers moyens de diffusion dans un ensemble de plus en plus complexe qui caractérise les sociétés modernes ${ }^{14} »$. 


\section{Le public des émissions littéraires} général (comme pratique de loisir alternative) mais des émissions littéraires (comme autre pratique culturelle), le rapport entre lecture et audience s'inverse : il n'est plus d'exclusion mais de renforcement. Ainsi, plus ils possèdent de livres et plus les Français regardent les émissions littéraires à la télévision (plus régulièrement aussi ils lisent une revue spécialisée). En effet parmi ceux qui n'en possèdent aucun, et de un à vingt-cinq livres, ils sont $60 \%$ et $53 \%$ à ne jamais regarder une émission littéraire, tandis que parmi ceux qui possèdent de cent à deux cent cinquante, et plus de deux cent cinquante livres, ils sont $15 \%$ et $19 \%$ à regarder souvent, et $24,3 \%$ et $24,8 \%$ à regarder de temps en temps une émission littéraire ( $c f$. tableau B en annexes).

L'assiduité aux émissions littéraires n'est alors qu'une modalité d'un investissement culturel cumulatif; la présence d'équipements et autres biens culturels au foyer étant également liée à la possession et au nombre de livres possédés, ( $c f$. tableau $C$ en annexes). Par exemple, les possesseurs de plus de deux cent cinquante livres sont $88 \%$ à posséder des disques (les non-possesseurs de livres sont 31,2\%), 47,9\% à avoir des reproductions d'œuvres d'art célèbres (3,4 \% chez les non-possesseurs), et 74,4 \% à être abonnés à un ou plusieurs revues ou journaux (contre $38 \%$ ).

En fait de rivalité livre-télévision, la présence du livre semble prédisposer à l'écoute des émissions littéraires. Certes, mais possession ne veut pas dire lecture et l'existence d'une bibliothèque peut tout aussi bien n'être que l'héritage d'une pratique lectorale passée, révolue et dont l'écoute des émissions littéraires tiendrait lieu aujourd'hui.

Or précisément, l'audience des émissions littéraires semble également être corrélée avec l'intensité de la lecture: que les non-lecteurs et les faibles lecteurs soient les téléspectateurs auprès de qui l'audience est la plus faible n'est pas pour nous surprendre. Ce ne sont pas néanmoins les plus "gros » lecteurs (cinquante livres et plus lus dans l'année) qui sont les plus assidus. C'est au-delà de quinze livres lus dans l'année que l'audience de ce type d'émission est la plus forte, et particulièrement dans la tranche des lecteurs de vingt-cinq à quarante-neuf livres par an.

Notons d'ailleurs que le phénomène s'est accentué depuis 1973 à la faveur notamment d'une plus grande assiduité aux émissions littéraires rendue possible par une augmentation du taux de possession de la télévision ( $c f$. tableau D en annexes).

Pour autant, si l'on considère le taux de progression de l'assiduité aux émissions littéraires (calculé sur la base de ceux qui regardent « au moins de temps en temps »), entre 1973 et 1981, la thèse de la corrélation avec la lecture se trouve singulièrement remise en cause. Il apparait, en effet, que cette assiduité accrue est paradoxalement le fait des faibles lecteurs et des non-lecteurs !

Voici qui doit nous amener à reconsidérer l'argument communément admis d'un public spécifique pour les émissions littéraires, qui partagerait les mêmes caractéristiques socio-démographiques distinctives des possesseurs, des acheteurs et des lecteurs de livres. Il importe alors de différencier deux modalités de mesure de l'audience.

50 Si l'on considère la proportion des téléspectateurs qui, au sein de chaque catégorie socio-démographique, regarde "souvent» ou «de temps en temps» les émissions 
littéraires, on ne peut manquer de noter une forte homologie avec les données correspondantes concernant les pratiques du livre (possession, achat et lecture). C'est dans le même sens que les variables «CSP ", «taille de l'agglomération » et surtout «diplôme de fin d'études» déterminent le niveau de la pratique. Dans chacun des cas, l'intensité de la pratique est la plus forte chez les «cadres supérieurs et professions libérales » et au contraire la plus faible chez les « agriculteurs » et les «OS, manœuvres, personnels de service». Elle augmente en fonction de l'accroissement de la taille de l'agglomération, et de manière plus spectaculaire encore en fonction du niveau du diplôme.

51 Ajoutons que ces caractéristiques partagées par les téléspectateurs des émissions littéraires et avec les possesseurs, acheteurs et lecteurs de livres, les distinguent par ailleurs des téléspectateurs des autres émissions.

Si l'on considère maintenant non plus la proportion des téléspectateurs qui, au sein de chaque catégorie socio-démographique, regarde souvent ou de temps en temps chaque genre d'émission, mais pour chaque genre, la structure de son audience comparée à celle de l'ensemble des téléspectateurs, les résultats apparaissent beaucoup plus contrastés. D'une part, les caractéristiques relevées précédemment se confirment effectivement, le public des émissions littéraires comprenant en particulier le plus fort pourcentage de "cadres supérieurs et professions libérales", de "bacheliers » et «titulaires de diplômes de l'enseignement supérieur ».

Ces caractéristiques générales sont au demeurant confirmées par des données récentes concernant le public d'Apostrophes. «[Il] est plus féminin que celui de la télévision le vendredi à cette heure-là $(58 \%$ contre $53 \%$ ) et plus urbain ( $49 \%$ de son auditoire réside dans une agglomération de plus de 100000 habitants contre $43 \%$ ). Les téléspectateurs, fidèles à l'émission, parmi les actifs, sont plutôt des cadres ( $8 \%$ contre $4 \%$ de l'ensemble des téléspectateurs), des membres de professions intermédiaires (11\% contre $9 \%$ ) et des petits patrons ( $4 \%$ contre $3 \%$ ). De plus, le quart de ce public a suivi des études supérieures (contre $14 \%)^{15}$. »

Cependant, on note d'autre part certains traits qui, sans être spécifiques, sont néanmoins tout autant caractéristiques de ce genre d'émission. En particulier la forte représentation des «inactifs" ("plus de soixante ans » et plus encore «femmes de moins de soixante ans »), la part non négligeable des «OQ » et «OS », et surtout, le plus fort pourcentage de "cadres moyens». Tandis que les téléspectateurs des communes de moins de 100000 habitants constituent près de la moitié du public, et les nontitulaires du baccalauréat près des deux tiers!

Tout compte fait, s'il n'est guère surprenant de retrouver les familiers du livre dans le public des émissions sur les livres, il est plus étonnant en revanche de constater qu'ils représentent à peine la moitié de l'audience. Procéder à l'analyse du public des émissions littéraires suppose donc pour le moins une attention égale à chacun de ces deux aspects. Or, de manière générale, c'est loin d'être le cas, les études statistiques, dans leurs présupposés aussi bien que dans leurs commentaires, contribuant à objectiver l'existence d'un public spécifique aux émissions littéraires, dans le cadre d'une problématique implicite de la domination culturelle comme domination de classe. Exemplaire de ce point de vue, ce commentaire d'un tableau portant sur la composition de l'auditoire des quatre émissions de la série Italiques de janvier 1972 dans le panel-télévision, comparée avec celle de l'ensemble des personnes interrogées : « cet auditoire compte proportionnellement plus de personnes de niveau d'instruction 
« supérieure » $(+13 \%)$, plus de cadres supérieurs $(+5 \%)$, plus de personnes habitant la région parisienne, $(+10 \%)$, plus de personnes de plus de 50 ans et enfin plus de femmes $(+8 \%)^{16} »(c f$. tableau $\mathrm{E}$ en annexes).

56 La thèse du public spécifique relève ici d'une interprétation abusive et constitue une véritable imposition de sens. D'une part, au lieu de rapporter la composition du public d'Italiques à celle d'autres émissions, on compare des pourcentages mesurant une pratique effective à des pourcentages qui tiennent à la répartition des différentes catégories dans la population française (dont l'échantillon du panel est, par construction, représentatif). Si bien que l'on ne peut déterminer la nature de l'écart enregistré : rien n'atteste ici qu'on ne retrouverait pas par exemple une aussi forte proportion de femmes ou de personnes de plus de 50 ans dans le public d'autres émissions, et que ce qui est pointé comme spécifique d'une émission particulière ne s'applique pas au public de la télévision en général. D'autre part, on note la surreprésentation des personnes dotées d'un niveau d'instruction supérieure, on ne relève pas que plus du tiers du public d'Italiques est composé de personnes de niveau d'instruction primaire!

\section{L'impact des émissions littéraires}

Où il apparaît que la légitimation culturelle de la télévision établie par la reconnaissance de l'impact des émissions littéraires se paie d'une double méconnaissance: celle des pratiques du livre comme pratiques lectorales, celle des pratiques de la télévision comme pratiques spécifiques.

Si le public des émissions littéraires ne se réduit pas au cercle des familiers du livre, s'il convient en d'autres termes de ne pas «absolutiser » la corrélation entre pratique du livre et écoute des émissions littéraires, à plus forte raison, faut-il se garder de surestimer leur impact sur un tel public, et en premier lieu, de tout raisonnement par inférence. Établir que ceux qui possèdent ou lisent le plus grand nombre de livres regardent proportionnellement davantage que les autres les émissions littéraires signifie simplement que les pratiques du livre sont un déterminant important de cette écoute (mais pas le seul); et n'implique aucunement un effet en retour de l'écoute sur la possession ou la lecture de livres. Est-ce à dire, en soulignant de la sorte les dangers de l'inférence, que nous ne disposions pas de données spécifiques pour évaluer l'effet des émissions littéraires sur la pratique lectorale? Une étude du CЕO de 1984 apporte pourtant des résultats significatifs relatifs à l'achat de livres présentés dans une émission de télévision ${ }^{17}$ ( $c f$. tableau $\mathrm{F}$ en annexes).

Il ressort, en effet, que plus on regarde les émissions de télévision sur les livres, plus on achète les livres qui y sont présentés. En outre, quand ceux qui regardent les émissions de télévision sur les livres achètent, c'est plutôt plusieurs livres qu'un seul. Enfin, plus on regarde les émissions de télévision sur les livres, plus on achète plusieurs livres ${ }^{18}$.

Pour autant, le sens de la relation de causalité reste indécidable : on peut tout autant bien penser que ce sont ceux qui consomment le plus de livres qui regardent le plus souvent les émissions sur les livres. Sans compter que $72 \%$ du public de ces émissions (et $45 \%$ de ceux qui les regardent souvent !) n'achètent pas de livres... Alors, les émissions de télévision sur les livres font-elles vraiment acheter des livres?

61 A défaut de réponse, une enquête de 1976, intitulée "Le livre et l'information » menée par l'ARc.mc à la demande de l'Association des attachés de presse de l'édition ${ }^{19}$ nous 
fournit des éléments de comparaison avec les autres médias. Or, sur ce point si $57 \%$ des lecteurs considèrent que l'information sur les livres à la radio, à la télévision et dans les journaux incite à la lecture (tandis que $41 \%$ pensent le contraire), c'est l'impact de la presse écrite qui s'avère être le plus déterminant. $37 \%$ déclarent en effet avoir lu ou acheté un livre, au cours des douze derniers mois, «après avoir lu dans un journal ou une revue, un article en signalant l'existence », $30 \%$ « après avoir entendu parler de ce livre dans une émission de télévision » et $17 \%$ « après avoir entendu parler de ce livre dans une émission de radio ».

Cependant, il s'en faut de beaucoup que pour chaque média, l'impact des informations littéraires (proportion de lecteurs qui y trouvent cette catégorie d'informations) soit égal, voire même proportionnel à son audience auprès des lecteurs de livres. ( $C f$. tableau $\mathrm{G}$ en annexes).

La presse périodique, bien qu'elle touche régulièrement un nombre moins important dé lecteurs de livres, se caractérise néanmoins, du point de vue des informations littéraires, par un impact égal en valeur absolue à celui de la presse quotidienne ou de la télévision, et par un impact nettement supérieur en valeur relative.

On peut en conclure que la presse périodique remplit plus spécifiquement que les autres médias son rôle d'information sur les livres. Soit qu'elle ait une vocation particulière en ce domaine, soit que les lecteurs de livres qui lisent des revues et magazines s'intéressent plus à l'information littéraire que ceux qui lisent la presse quotidienne, écoutent la radio ou regardent la télévision.

Par ailleurs, les informations sur les livres susceptibles d'être délivrées par les différents médias ne sont pas tenues pour équivalentes d'un point de vue formel. Les acheteurs (lecteurs) de livres préfèrent le plus souvent recevoir, dans la presse, l'information sur les livres sous des formes classiques et traditionnelles. C'est d'abord une critique détaillée analysant le contenu du livre, son intérêt, ses qualités et ses faiblesses $(37 \%)$; ce sont aussi des informations brèves sur le sujet du livre, son intérêt, le nom de l'auteur et de l'éditeur, son prix (36\%). Viennent ensuite : une interview de l'auteur (24\%), un reportage sur le sujet du livre $(23 \%)$; la publication de passages les plus significatifs $(21 \%)$; des commentaires de personnalités ou de spécialistes du sujet (17\%) ; un classement des meilleures ventes (8\%).

En revanche, les débats, rencontres-discussions et reportages sont les formes privilégiées de l'information sur les livres à la télévision; elles l'emportent de loin sur la trop classique interview de l'auteur. Les téléspectateurs se prononcent, en effet, par ordre de préférence, pour un débat entre l'auteur, des journalistes, des personnes concernées par le sujet du livre, éventuellement d'autres écrivains (37\%). Quand le sujet s'y prête (par exemple livre sur l'art, l'histoire, certains grands hommes, etc.), ils se prononcent pour un reportage sur le sujet du livre, avec documents photographiques, cinématographiques, etc. $(35 \%)$; une rencontre-discussion entre un auteur et des lecteurs ( $34 \%)$; une interview de l'auteur chez lui, dans son cadre de vie habituel ( $26 \%)$ ou faite, en studio, par un grand journaliste spécialisé de la télévision (16\%).

67 A proprement parler, les téléspectateurs intéressés par les livres attendent de la télévision non pas tant des informations finalisées, centrées sur le livre (susceptibles de leur permettre d'en apprécier la qualité littéraire et donc de les orienter dans leur choix de consommateurs). Ils en attendent plutôt des commentaires, sur le thème traité par le livre dont l'intérêt n'est pas fonction de la qualité du livre. Autrement dit, la 
présentation du livre même, dans les modalités attendues de la télévision, y est moins étroitement finalisée. Sans nous prononcer ici sur l'impact respectif des différents types de traitement de l'information littéraire susceptibles de provoquer l'achat et $a$ fortiori la lecture des livres présentés, notons que cette diversité des types en encourage l'usage cumulatif. Ce qui remet en cause la validité des sondages et autres enquêtes statistiques visant à hiérarchiser les différentes sortes de prescripteur du livre à partir du présupposé de leur exclusion mutuelle, et dont l'enquête "Le livre et l'information " fournit un exemple. Ainsi, $37 \%$ des lecteurs interrogés (près de 9,8 millions de Français) avaient lu ou acheté au cours des douze mois précédant l'enquête certains livres donnés après avoir lu, dans un journal ou une revue, un article qui les leur avait signalés ; $30 \%$ (près de 8 millions), après avoir entendu parler de ces livres dans une émission de télévision ; $17 \%$ (4,5 millions environ), après en avoir entendu parler à la radio. » (cf. tableau $\mathrm{H}$ en annexes)

Significatifs, ces pourcentages le sont tout au plus si l'on oppose le nombre restreint d'émissions littéraires à la multiplicité des titres de la presse écrite; mais ce dont ils donnent alors implicitement la mesure c'est simplement celle de l'uniformisation résultant de la prescription télévisuelle.

Dénominateur commun des différentes tentatives pour évaluer statistiquement l'impact des émissions littéraires : c'est à l'achat qu'est rapportée l'influence supposée des émissions sur les livres. Ce point est d'importance pour qui interroge les conditions de production des données statistiques relatives aux rapports qu'entretiennent la télévision et le livre ainsi que les modalités de pertinence de leur usage sociologique. Il révèle, en effet, l'arrière fond tacite qui légitime ce type de questionnement: la production même des données statistiques participe implicitement du même modèle distributif qui prévaut dans « la sociologie de la lecture » et s'expose donc à la même critique que nous avons déjà formulée à son encontre ${ }^{20}$.

70 "Ce qui d'ordinaire tient lieu d'approche de la lecture, c'est en effet une analyse de la distribution sociale des pratiques de l'usage du livre, comme pratiques culturelles socialement différenciées en fonction du niveau d'instruction et de l'origine sociale, et objectivement repérables (modes d'information et d'acquisition des ouvrages, temps consacré au livre, genres possédés...). L'acte de lecture proprement dit, quant à lui, lorsqu'il n'est pas tout simplement évacué, est au plus inféré de ces deux variables déterminantes. En d'autres termes, l'acte de lecture n'est paradoxalement pas l'objet de cette "sociologie de la lecture" qui se préoccupe en fait de mesurer l'efficacité pratique de l'imposition d'une pratique d'usage légitime des productions culturelles. »

71 Au-delà encore de la mise à jour des implicites attaches aux modalités de la mesure, il convient de remettre en cause la présupposition même d'un impact. "Si l'on se préoccupe tant des effets de la télévision, remarquent opportunément $\mathrm{P}$. Chambat et $\mathrm{A}$. Ehrenberg, c'est qu'elle est prisonnière d'un schéma culturel qui appréhende le téléspectateur comme consommateur amorphe, malléable ou passif, simple support de préférences, comme sujet transparent, sans épaisseur et à la limite sans existence. Cette explication recouvre une représentation du public comme masse, ancrée dans la définition même du média. Une sociologie spontanée de la masse tient lieu de réflexion sur les usages de la télévision ${ }^{21} »$ D'autre part, le type d'efficacité supposée des émissions sur les livres relève d'une acception instrumentale des médias, conforme à la théorie de l'information: un message est diffusé par un émetteur à destination d'un public-récepteur, dans une séquence de type stimulus-réponse. Cette représentation du 
média autorise un principe de mise en équivalence, tant des différents médias (dont on peut comparer l'efficacité respective) que des différentes émissions. Ainsi on peut tout aussi bien, et de la même manière, comparer l'impact des émissions sur les livres ou des émissions sur la cuisine... le pourcentage des téléspectateurs non influencés étant d'ailleurs le même dans les deux cas !

\section{Les émissions littéraires : des émissions (presque) comme les autres}

72 Où il apparaît que la redéfinition de la pratique télévisuelle comme pratique culturelle à part entière est indissociable de la remise en cause de ses anciennes modalités de mesure.

73 Si le public des émissions littéraires déborde le cadre des familiers du livre et si, par là même, la lecture (ou, tout au moins l'achat) ne finalise que partiellement l'écoute, il faut considérer enfin les émissions littéraires comme des émissions spécifiques. C'est-àdire non plus du point de vue de la spécificité de leur public ou de leur effet sur les ventes, mais en tant que leur écoute constitue une pratique propre pour un public au contraire élargi.

Rappelons, en effet, que parmi les différentes catégories d'émissions, les émissions littéraires sont les seules à avoir élargi leur audience (tout en conservant la même place dans la hiérarchie). Elle est passée de $30 \%$ à 38,8\% entre 1973 et 1981 ; un nombre croissant de téléspectateurs regrettant même qu'elles ne soient pas "programmées plus souvent et en début de soirée ${ }^{22}$ ». A l'origine de cet accroissement spectaculaire d'audience, la création en 1975 d'Apostrophes, soit l'invention d'une nouvelle formule de débat télévisé entre des auteurs réunis sur le plateau. Il n'est pas dans notre propos ici de nous interroger à notre tour sur les raisons du succès d'Apostrophes ${ }^{23}$ mais simplement de l'apprécier du point de vue des goûts et préférences du public en matière de programmes; de considérer en bref le véritable plébiscite dont elle est l'objet. Apostrophes arrive en effet en tête des émissions les plus appréciées, $95 \%$ des téléspectateurs déclarant avoir du plaisir à la regarder, et $97 \%$ qu'elle est une émission de très grande qualité ${ }^{4}$. En réponse à une question ouverte, dans la même enquête, Apostrophes est encore considérée par $33 \%$ des téléspectateurs comme la plus culturelle des émissions (Océaniques, qui arrive en deuxième position, ne recueillant pour sa part que $5 \%$ des suffrages); et last but not least elle est sacrée meilleure émission de télévision ${ }^{25}$. Cependant (et même si ce pourcentage est le plus faible parmi les émissions qui ne sont pas diffusées à $20 \mathrm{~h} 30$ ), la même enquête Ipsos nous indique que $38 \%$ des personnes interrogées ne regardent jamais Apostrophes! Voilà qui est clair, la réputation de l'émission dépasse largement son audience. En d'autres termes, ce dont il est question ici, ce n'est plus de pratiques mais de représentations. La note de synthèse du Service des études et de la recherche d'Antenne 2 qui analyse les principales conclusions des enquêtes que l'on vient de citer, en apporte la confirmation: elle oppose, au succès d'audience de TF1, une " supériorité d'image incontestable d'Antenne $2 »$; le plébiscite d'Apostrophes ayant pour effet de doter la chaîne du label culturel.

75 Si TF1 est classée première dans la catégorie « films ", « séries ", "variétés » et " jeux », Antenne 2, elle, obtient la première place en ce qui concerne le sport» et les "magazines culturels». A cet égard, Apostrophes est une émission de référence "car elle génère le sourire, l'humour et la complicité ", témoignant ainsi que la vocation 
d'une chaîne n'est pas d'être soit culturelle, soit commerciale, et par là que « le culturel ne doit jamais être ennuyeux ".

76 Au-delà de la simple (!) mesure des pratiques du livre et de la télévision, accéder aux représentations qui gouvernent le rapport entre télévision et culture, voilà précisément ce que vise un récent sondage ${ }^{26}$. Il pose de nouveau, dans un contexte, cette fois, de multiplication des chaînes, la même question « La télévision, entrave à la lecture?", déjà posée dans les années soixante, dans un contexte de diffusion généralisée des postes de télévision. Dans la logique qui fut la nôtre dans le compte rendu critique des données statistiques sur le rapport qu'entretiennent la lecture et la télévision, ce présent sondage, dont nous rapportons ci-après les commentaires les plus significatifs, préfigure un nouveau type de problématisation, associé à une nouvelle transformation du champ télévisuel, radicalement distinct des précédents problématiques de la concurrence puis des effets induits - qui, eux, partageaient une même focalisation sur les pratiques.

77 «Si globalement, l'écrasante majorité des interviewés (72\%) estime que la multiplication des chaînes de télévision n'a rien changé à leurs habitudes de lecture, on constate la prégnance du stéréotype selon lequel la télévision inhiberait la lecture.

78 «Deux indicateurs l'attestent. Lorsque les interviewés reconnaissent à la télévision une incidence sur leurs habitudes de lecture, il s'agit d'abord d'une diminution de ces pratiques : $18 \%$ des interviewés pensent en effet avoir moins de temps à consacrer à la lecture contre $5 \%$ d'entre eux qui estiment que la multiplication des chaînes constitue une incitation à lire davantage. De même lorsqu'ils se prononcent sur l'influence de la télévision sur la lecture des enfants, $68 \%$ des interviewés estiment que la télévision constitue un frein à la lecture, tandis qu'ils ne sont que $14 \%$ à reconnaître au petit écran une fonction d'éveil de la curiosité enfantine. »

79 On ne saurait être plus clair, la question toujours répétée des rapports du livre et de la télévision a produit ses propres stéréotypes: ceux-là mêmes qu'enregistrent les enquêtes et autres sondages, en fait de mesure des pratiques objectives. "C'est un roman de science-fiction dans lequel nous sommes plongés depuis une trentaine d'années déjà : la guerre des mondes entre la galaxie Gutenberg et la galaxie Mac Luhan. »Et ces procédures d'objectivation, d'institution de la réalité que sont les enquêtes statistiques et autres sondages d'opinion, loin d'opérer dans une stricte extériorité, en constituent proprement les chapitres successifs; en sorte qu'elles risquent fort de ne mesurer en fait que l'efficacité de leur propre activité d'imposition. D'ailleurs, n'est-ce pas par une citation empruntée au Métier de sociologue que s'achève cette récente analyse critique du concept d'audience ${ }^{27}$ ?

80 «Les usages sociaux liés aux médias évoluent, tout comme la société et les médias. La notion d'audience mérite donc une lecture critique pour résister à la trompeuse évidence d'un objet défini une fois pour toutes, dont il ne resterait plus qu'à améliorer la mesure par la mise au point de méthodes supposées de plus en plus fines. Le problème de fond n'est pas tant de perfectionner la méthodologie des mesures, que de prendre conscience que la "familiarité" avec laquelle tout le monde parle aujourd'hui d'audience, du chercheur au journaliste, est "l'obstacle épistémologique par excellence" ", parce que cette "familiarité produit [...] des conceptions ou des systématisations fictives en même temps que les conditions de leur crédibilitée ${ }^{28}$."

81 Ainsi, les enquêtes quantitatives d'audience actuelles (en dépit de leur sophistication croissante : audimat, système de bouton-poussoir, etc.) sont-elles vouées à rester à la 
périphérie du phénomène qu'elles prétendent mesurer, ne mesurant effectivement qu' " un artefact qui permet la commercialisation des audiences». Sauf à s'engager sur la voie d'une « phénoménologie des audiences » qui reconnaisse enfin le téléspectateur comme un équivalent de ce qu'est le lecteur dans les recherches sur l'esthétique de la réception: non pas un récepteur passif voire fasciné mais un acteur de la communication, engagé dans une activité de réception. A l'heure où d'aucuns annoncent l'avènement d'une "culture de l'écran", des instruments de mesure renouvelés sont donc en passe d'établir une nouvelle réalité des pratiques. La distinction générique livre-télévision est révolue ; voici venu le temps de l'analogie.

Cependant, alors même que des techniques de mesure adaptées s'apprêtent à instituer la pratique télévisuelle en pratique lectorale, qu'en est-il encore de la lecture ? Est-elle bien cet invariant supposé auquel rapporter toujours des pratiques télévisuelles dont on reconnait, en revanche, les transformations? Et si la télévision avait elle-même opéré une transformation profonde de la lecture ; non pas simplement de son intensité mais du sens même qui lui est attaché ? Et s'il fallait paradoxalement reconsidérer la pertinence de la question des « effets de la télévision sur la lecture » en la posant cette fois au niveau non plus de la pratique mais du sens de la pratique?

\section{NOTES}

1. Sur ces trois modalités d'examen, $c f$. J. Schlanger. Penser la bouche pleine, Fayard, 1983. Au niveau de l'examen critique où nous nous plaçons, il n'y a pas lieu de hiérarchiser les différents types de sondage, pourtant fort hétérogènes du point de vue de leur fiabilité, ce qui équivaudrait pour nous à mesurer la professionnalité des divers «faiseurs de sondage ». Du point de vue qui est le nôtre, un sondage Louis-Harris vaut bien un sondage Insee !

2. Cf. Livres-Hebdo du 29 septembre 1987, "Le temps de la lecture et de ses concurrents en minutes » (souligné par nous).

3. Ce sont, en effet, toujours les jeunes qui lisent le plus. Et de loin. Le pourcentage des gros lecteurs (moyenne nationale : $22 \%$ ) s'élève à $32 \%$ dans la tranche d'âge des 18-24 ans, pour redescendre progressivement jusqu'à $16 \%$ chez les 50-64 ans. La "génération Marconi", sollicitée par la télévision, mais plus instruite, lit autant que les “générations Gutenberg”. " (L'Express, 4 novembre 1975).

4. P. Chambat, A. Ehrenberg, «La culture de l'écran », Le Débat, n 58, 1988.

5. Données dont la production même, antérieure à la mise en place du Centre d'étude d'opinions (CEO), témoigne de la précocité du problème posé par le rapport lecture-télévision, et de l'intérêt qu'il a aussitôt suscité.

6. Organisée à l'occasion de la première semaine nationale de la lecture en 1967 par la jeune chambre économique de la région de Saint-Etienne et l'association « Lire».

7. Invités par les enquêteurs de l'Ires à apprécier l'affirmation: «Ce sont les paresseux qui aiment la lecture ", $82 \%$ des interviewés protestent, et cela malgré l'ambiguïté du terme «lecture ».

8. R. Kaes, Images de la culture chez les ouvriers français, Cujas, 1968. 
9. J.-C. Passeron, présentation à R. Hoggart, La Culture du pauvre, Éd. de Minuit, 1970, p. 18 (rappelons que le texte original est paru en 1957).

10. A l'appui de cette thèse de l'invisibilisation, notons que c'est seulement en 1980 que des rapports officiels reconnaissent pour la première fois l'existence du problème de l'illettrisme. $C f$. J. Dumazedier, H. Gisors : Français analphabètes ou illettrés ?, Adrac, octobre 1984.

11. D'autres enquêtes plus récentes confirment ce pourcentage. Par exemple, un sondage effectué pour Madame Figaro, 2 juillet 1988, auprès d'un échantillon représentatif de l'ensemble de la population âgée de 18 ans et plus, donne les résultats suivants à la question «En général, qu'est-ce qui vous incite à lire un livre? » : le sujet du livre $71 \%$; vous appréciez son auteur $34 \%$; les émissions littéraires à la télévision, (Apostrophes, etc.) $24 \%$; les critiques que vous avez lues ou entendues sur le livre $24 \%$; les conseils d'amis ou de parents $16 \%$; sans réponse $4 \%$. (Le total des pourcentages est supérieur à 100, les personnes interrogées ayant pu donner plusieurs réponses. Lecteurs interviewés ayant lu au moins deux livres dans l'année.)

12. Si cette observation générale est bien confirmée par d'autres enquêtes plus récentes, celles-ci nous permettent cependant une évaluation plus nuancée de l'évolution des divers types de lecture. Ainsi l'enquête Insee sur l'évolution des loisirs entre 1967 et 1987 confirme que « dans la mesure où ils répondent à un besoin d'évasion que satisfont également les émissions télévisées de fiction ", les livres subissent à plein la concurrence de la télévision. " A tous niveaux de diplômes, la lecture des livres décroît. Et ce n'est que parce que les plus diplômés, qui sont aussi les plus gros lecteurs, sont devenus plus nombreux, que la proportion de ceux qui lisent au moins un livre par mois est restée stable, autour de $30 \%$. » Elle révèle en revanche que la proportion de ceux qui lisent le journal tous les jours est passée de $60 \%$ à $42 \%$. «Les jeunes surtout délaissent les quotidiens. Les agriculteurs et, plus généralement les habitants des communes rurales, résistent mieux que les autres; mais précisément, la concurrence de la télévision est ici moins forte puisqu'elle ne traite pas contrairement aux journaux régionaux, de nouvelles très locales ». Elle révèle également que le seul type de lecture à s'être développé est celui des magazines et des revues. « $79 \%$ de la population en lit régulièrement au moins un, contre $56 \%$ en 1967 . La concurrence de la télévision est sans doute moins forte ici. D'une part, le contenu des revues est souvent spécialisé, tandis que les émissions télévisées s'adressent de préférence au plus grand nombre. D'autre part, plus aisément que pour les livres, la lecture de revues peut être morcelée, sans pour autant que le contenu se périme rapidement comme celui des quotidiens : lus dans les interstices de la vie quotidienne, les magazines échappent mieux à la contrainte de temps que fait peser la télévision. » (O. Choquet, H. Valdelièvre : «Les loisirs 20 ans après : davantage de sorties et de télévision ", Premiers résultats, Insee, $\mathrm{n}^{\circ} 148,1988$.

13. 0 . Choquet, $\mathrm{H}$. Valdelièvre, op. cit.

14. G. Cazeneuve, «Le livre et la télévision », La Nef, n 61-62.

15. Mensuel $n^{\circ} 24$ de Médiamétrie, octobre 1988 ; sources: structure du public repéré par l'audience cumulée $21 \mathrm{~h}$ 30-23h 00 du vendredi entre janvier et juin 1988, enquête 55000.

16. Les émissions littéraires télévisées, SEO, 7 août 1972.

17. «Les émissions de télévision sur les livres - les émissions de télévision sur la cuisine». Enquête auprès d'individus âgés de 15 ans et plus, possesseurs de radio et/ou de télévision. CEO, janvier 1984.

18. On retrouve ces mêmes tendances dans un tableau des Pratiques culturelles des Français (1973), moins accusées cependant du fait que les pourcentages portent sur les possesseurs de téléviseur et non pas, spécifiquement, sur le public des émissions sur les livres.

19. Auprès d'un échantillon représentatif de la population française des lecteurs de livres âgés de 15 ans et plus (ayant lu, au cours des 12 derniers mois, au moins un livre, à l'exclusion des livres scolaires et des revues ou magazines).

20. M. Peroni, Histoires de lire, Éd. BPI, 1988, p. 8-9.

21. P. Chambat, A. Ehrenberg, op. cit. p. 116. 
22. Pratiques culturelles des Français. Évolution 1973-1981, Dalloz, 1982.

23. Il ne sera pas non plus question ici du dit «effet Apostrophes» sur les ventes. Le lecteur intéressé trouvera dans l'ouvrage d'E. Brasey : L'Effet Pivot, Ramsay, 1987, p. 349, une corrélation entre meilleures ventes et passage à Apostrophes, pour la période 1983-1986.

24. Enquête Ipsos-Télé poche, octobre 1988, «Culturelle ou commerciale, quelle télévision pour les Français?»

25. En réponse à une question ouverte. Enquête Estel/Antenne 2, janvier 1989.

26. Sondage Sofres-Livre du mois, « Télévision et littérature », janvier 1988.

27. E. Fouquier, J.-C. Lioret, «Définitions du concept d'audience, analyse critique et orientations », in l'Audience et les médias, Irep, Les Éditions d'organisation, 1989.

28. P. Bourdieu, J.-C. Chamboredon, J.-C. Passeron, Le Métier de sociologue, Mouton, 1968, p. 27. 


\section{Chapitre 2. Le livre sur un plateau : la lecture figurée}

\section{Le livre à la télévision : une question d'actualité}

1 Avant même d'entreprendre le repérage et l'analyse des diverses modalités de la présence du livre à la télévision dont nous avons bien hâtivement annoncé la pertinence, comme si cette présence allait de soi, nous voudrions mettre l'accent sur le paradoxe de cette présence même, en prémisse à notre re-problématisation de l'objet. Que la présence du livre à la télévision soit paradoxale, c'est ce que révèle en effet une réflexion sur la spécificité du média, notamment quant au rapport qu'il entretient avec la réalité. Ainsi, L. Quéré et É. Véron, pour ne citer qu'eux, s'accordent à voir l'efficacité du message télévisuel dans la construction performative d'une réalité par illusion référentielle ${ }^{1}$. Le temps télévisuel en particulier n'est autre que celui de l'actualité, au double sens de saisie immédiate d'un réel référentiel («les actualités») et d'immédiateté entre présent de l'énonciation et présent de la réception. Il est celui de l'action en train de se faire (action dramatique: les séries... aussi bien qu'action discursive: Alain Decaux raconte...), de la performance (dont le «direct» est la manifestation par excellence). Or, précisément, parce qu'elle remettrait en cause l'immédiateté de l'énonciation (la lecture comme actualisation d'un énoncé déjà là), parce que la dimension de l'action lui fait défaut (la lecture comme expérience intime) ${ }^{2}$, la lecture ne saurait proprement avoir de place à la télévision.

2 De fait, la pratique de la lecture, d'ordinaire inavouable, n'a d'autre visibilité télévisuelle que dans les creux d'un dispositif technique destiné à produire la fiction d'une non-lecture. Exhibée, lorsque le présentateur se détourne du téléspectateur (du prompteur) pour donner lecture d'une dépêche qui vient de tomber, elle devient paradoxalement opérateur d'immédiateté : «J'en prends connaissance en même temps que vous. »

3 Si la présence télévisuelle du livre est par principe détachée de l'acte de lecture, au moins peut-elle témoigner d'une pratique d'écriture, se redoublant pour ce faire dans la présence, non moins problématique, de l'écrivain, comme tenant lieu de son livre. L'écrivain est alors supposé venir, à la place du livre, dire ce qu'est le livre : « un genre, 
un contenu, un style, une critique, bref un résumé, c'est-à-dire aussi bien tout ce qu'il n'est pas exactement, ou plutôt tout ce qui ne vaudrait pas la peine qu'il soit s'il n'était que cela... Ceci est le livre, voici l'écrivain, chacun devient la garantie de l'autre, mais cette garantie ne signifie rien sinon une tautologie ${ }^{3}$. »Certes, une telle opération établit la preuve positive d'existence du livre aussi bien que de l'écrivain; mais d'un livre moins la lecture, c'est-à-dire un fétiche, mais d'un écrivain moins l'écriture, c'est-à-dire une désignation sociale ${ }^{4}$. Autrement dit, quand la lecture du texte est exclue par principe du cadre de l'action télévisuelle, la parole de son auteur, supposée pouvoir s'y substituer, sort pour sa part du cadre du projet littéraire.

4 Faire de l'écrivain le tenant lieu de son livre «suppose en effet que l'écrivain ayant écrit (un texte, un livre), il lui reste encore quelque chose à dire : quoi ? Des oublis ? Des déchets? A moins que (c'est le cas courant) on ne lui demande de se redire, ou, ce qui est pire, d'énoncer sous une forme claire ce qu'il a écrit sous une forme ramassée (mais l'ambiguïté du sens, des sens, l'ellipse, l'amphibologie, la figure, le jeu de mots, l'anagramme, qui constituent l'écriture, ne sont pas des faits de style ; c'est plutôt une pratique dénonciation qui engage le sujet aux prises avec le langage.) L'écriture est précisément ce qui excède la parole, c'est un supplément où s'inscrit, non pas un autre inconscient (il n'y en a pas deux) mais un autre rapport du locuteur (ou de l'écouteur) à l'inconscient. Donc la parole ne peut rien ajouter à l'écriture. Ce sur quoi j'ai écrit m'est dès lors interdit de parole : que dirais-je de plus ou de mieux? Il faut bien se persuader que la parole est toujours en arrière de l'écriture (et donc de la vie privée, qui n'est que le déploiement d'une parole : je suis toujours, par statut, plus bête, plus naif, etc., que ce que j'écris : je ne suis pas comme j'écris). Le seul genre d'entretien que l'on pourrait à la rigueur défendre, serait celui où l'auteur serait sollicité d'énoncer ce qu'il ne peut pas écrire ${ }^{5}$ ".

5 L'interdiction de parole énoncée par Barthes semble concerner au premier chef les auteurs de fiction, les «littérateurs ». Ainsi, le romancier M. Rio, argumentant son refus de se rendre à l'émission Apostrophes pour son dernier ouvrage, revendique-t-il la spécificité du monde de l'imaginaire face à celui des idées.

Ce qui est de l'ordre du savoir peut se résumer, s'échanger avec précision, se discuter avec profit. L'imaginaire, lui, est quelque chose de solitaire par nature. L'idée même de "collègue" en littérature est à mes yeux un non-sens. Un écrivain est pour moi une syntaxe distinctive, une musique originale, un univers particulier, tout cela appelant un commentaire isolé. Mettez ensemble plusieurs scientifiques, il en sortira probablement quelque chose. Faites de même avec des écrivains, chacun perdra nécessairement sa substance au profit du plus bas niveau de la discipline collective (rendre compte d'une série d'anecdotes) ou de l'intérêt privé (se vendre à tout prix), ce qui exclut a priori la plus infime possibilité de parler de la seule chose dont il devrait être question : la littérature. Voilà pourquoi, selon moi, l'émission Apostrophes n'est convaincante que lorsqu'elle s'écarte de sa vocation initiale de manifestation littéraire. Le savoir peut et doit parfois se vulgariser, l'imaginaire non, sous peine de devenir vulgaire. Et dans ce dernier domaine, la vulgarité, c'est précisément le collectif. Ceci, qui constitue à l'évidence la valeur spectaculaire, massive et, si l'on peut dire, monétaire, des Apostrophes purement littéraires, représente à mes yeux l'uniformité, l'ennui, la veulerie et la sottise. J'ai la vanité de croire à mon individualité littéraire, la naïveté d'avoir une morale et la fraîcheur de m'y tenir, la chance d'avoir aux États-Unis), ce qui m'écarte du rôle plutôt pesant et ridicule de " poète maudit. En été dès le début favorisé par les médias (en France comme conséquence, je ne me déplace que si j'ai la certitude d'être seul et de pouvoir parler de littérature ${ }^{6}$. 
Reste que le bien-fondé de la médiatisation du débat intellectuel est pour sa part également l'objet de violentes critiques, stigmatisant la banalisation et la mise en équivalence médiatiques des idées et des valeurs. Sans nous engager dans la controverse, nous voulons simplement indiquer que la parole de l'homme de science ou de savoir n'est pas moins problématique que celle de "l'homme de lettres ». C'est ce que le philosophe Emmanuel Levinas laisse entendre après avoir expliqué simplement en quoi sa philosophie n'est pas une philosophie de l'être : «Il m'est arrivé de le dire $\operatorname{mieux}^{7} » !$

7 Paradoxale, la présence télévisuelle de l'écrivain l'est d'emblée, dès ses premières apparitions, aux origines même du média. Alors, le visage des écrivains n'étant pas connu, leur voix à peine entendue, leur seule présence à la télévision pouvait constituer un événement sur le mode de la révélation. C'est ainsi que le public de Cinq colonnes à la une a pu découvrir en 1961 le type même du vieux sage, à longue barbe blanche, en la personne de Gaston Bachelard. Le dispositif de l'entretien, dont nous reproduisons ciaprès quelques fragments, avec un plan constant sur le philosophe et des questions en voix off, marque d'ailleurs l'extrême déférence.

Question - Est-ce qu'il faut vous appeler maître?

[...]

Q. - Est-ce qu'il y a une définition du philosophe?

[...]

Q. - On pense généralement que le philosophe ne sert à rien...

[...]

Q. - Est-ce que le philosophe est supérieur aux autres hommes?

[...]

Q. - On a l'impression que le philosophe sait mieux vivre, qu'il est à l'abri des...

G. B. - Des quoi ? mais non! Vous voulez absolument me coller une étiquette de philosophe éloigné des difficultés matérielles. Ainsi par exemple, je sais tout faire, je sais faire la cuisine...

Et vous, est-ce que vous vous occupez des questions matérielles?

Q. - Moins que vous je le crains... au fond, c'est un art de vivre, Monsieur

Bachelard!

G. B. - C'est beau « art de vivre »!

8 L'entretien porte ensuite plus particulièrement sur le dernier ouvrage de Gaston Bachelard: La Poétique de l'espace.

G. B. - Où est-ce que vous êtes né, vous?

Q. - Je suis né à Montpellier.

G. B. - Oui, eh bien, vous habitiez une maison?

Q. - J'habitais, malheureusement, un appartement.

G. B. - Ben voilà, vous n'y connaissez rien.

[...]

Q. - Est-ce que c'est triste de vieillir, Monsieur Bachelard?

G. B. - Oui, quand même ; mais vous n'allez tout de même pas mettre ça !

En dépit des réponses, insignifiantes, aux questions posées, la présence même de Gaston Bachelard devant la caméra, avec toute sa personne, son visage, suffit à réactiver la figure impersonnelle et sans âge du vieux sage à barbe blanche. Personnage hors du commun, alors même que le philosophe n'a de cesse, se faisant à son tour questionneur, d'affirmer qu'il n'est pas si différent de nous : pour un vieux sage il est également triste de vieillir! Il n'y a qu'un pas entre la révélation et la dernière apparition, le document et l'archive, celui qui sépare le «vieux maître » du "grand homme disparu ». Car les « grands hommes » meurent aussi, fussent-ils à la manière de 
François Mauriac, «immortels» (i.e. académiciens). C'est alors à titre posthume que s'établit la présence, tout au moins virtuellement.

F. M. - Du moment que vous voulez avoir des documents sur moi, c'est que vous avez cette idée flatteuse que lorsque je ne serai plus là on fera, peut-être parfois, des petits rappels ${ }^{8}$.

10 A en juger par la concurrence actuelle dans la conquête d'une image médiatique, comme condition pour se placer dans le champ littéraire; à voir les écrivains d'aujourd'hui « occuper le terrain, mais ni pour des idées, ni pour un projet d'écriture, simplement pour être là ${ }^{9}$, le problème de la présence semble être définitivement classé (... aux archives INA!), supposé dès lors ne s'être posé comme tel qu'à des écrivains d'un autre âge confrontés à la modernité de la télévision. Et pourtant, il arrive encore à certains de thématiser cette double difficulté qu'il faut bien alors considérer comme inhérente à la présence de l'écrivain sur le plateau: d'une part, se détacher de son texte, c'est-à-dire faire acte de présence, et d'autre part, faire corps avec la réalité, c'est-à-dire faire la preuve de sa contemporanéité.

11 Que fait Vladimir Nabokov, en réponse aux questions posées dans cette émission en direct qui lui fut spécialement consacrée ? Il lit ! Il raconte des histoires préalablement rédigées, et qui nous font voir les questions elles-mêmes, empreintes d'une apparente spontanéité, comme des questions contraintes. Entre autres choses, il nous lit comment, au moment de devenir professeur de littérature russe aux États-Unis, il se découvre une incapacité absolue de parler en public, qu'il tentera de dissimuler en récitant son cours à la virgule près ${ }^{10}$ !

Que nous dit P. Guyotat, alors même que, probablement pour la première fois, une caméra nous fait assister au véritable travail d'écriture, et peut entrer dans la fabrique du texte (ce que permet ici la nature orale de son élaboration)? « Je suis comptable de cet univers, je ne veux pas le brader. On ne vit pas impunément avec un monde, on ne peut pas bien le définir; je ne sais pas, en tout cas c'est toujours scandaleux. C'est certainement plus facile de vivre dans un univers où il y a des êtres sur pied, c'est très difficile de vivre avec un monde comme ça, c'est très difficile de se présenter à la société11.»

13 Comment parler encore du livre dans ce no man's land qui se tient entre écriture et lecture? Au moment d'engager l'analyse, remarquons que Bernard Pivot rapporte paradoxalement le succès de son émission sur les livres à l'impossibilité de convoquer le livre lui-même.

B. P. - J'ai toujours pensé qu'une des raisons du succès de l'émission, en ce qui concerne la vente des livres, c'était la frustration. Je m'explique. 1. Les auteurs invités partent frustrés parce qu'ils ne se sont jamais exprimés autant qu'ils l'auraient voulu... 2. Après chaque émission, je suis frustré. Elle ne s'est jamais passée comme je l'aurais voulu. Eh bien! je soigne cette frustration en préparant le mieux possible l'émission suivante. 3. Enfin, le téléspectateur est lui aussi très frustré parce que l'émission ne lui a pas apporté tout ce qu'il en attendait. Elle s'est arrêtée trop vite, on n'a pas insisté sur telle idée, etc. Comment peut-il soigner sa frustration? Simplement en achetant un ou plusieurs livres présentés pendant l'émission et en les lisant.

Cahiers - Est-ce qu'elle n'est pas un petit peu mise en scène cette frustration?

B. P. - N'oubliez pas que vous assistez à un spectacle dont les principaux personnages sont finalement les livres, lesquels sont des parallélépipèdes qui ne bougent pas, ne parlent pas. Ils s'expriment, mais par délégation, grâce aux auteurs qui sont là et qui les représentent nommément. La frustration n'est pas organisée. 
Elle va de soi, elle est dans la nature même de l'émission et surtout, dans la nature

même des principaux invités d'Apostrophes : les livres ${ }^{12}$.

Comment parler du livre à la télévision? Depuis l'entretien les yeux dans les yeux de Lectures pour tous jusqu'au débat d'idées mis en scène sur le plateau d'Apostrophes, les formules se sont succédé au gré des émissions littéraires. Et pourtant la question demeure d'actualité (plus que jamais serait-on tenté de dire, si l'on considère d'une part la place privilégiée qu'occupe désormais la télévision dans les stratégies de promotion éditoriale, et d'autre part la toute puissance de l'audimat). Les réactions à la création récente d'Ex-libris et à l'annonce plus récente encore de l'arrêt d'Apostrophes nous le rappellent fort opportunément. C'est que les émissions littéraires, en tant que telles, sont prises dans une contradiction de principe : «littéraires ", elles ne peuvent l'être qu'à la condition d'accéder à la textualité des œuvres. Or, le temps télévisuel qui n'est autre que celui de l'actualité, du direct (de l'immédiateté entre le présent de l'action ou de renonciation et le présent de la réception) ne se prête pas à la lecture des textes. "Émissions " télévisées, elles doivent faire le spectacle à partir d'un objet inerte, le livre.

Nous nous proposons à présent de considérer la diversité des modes de convocation du livre et de l'auteur, comme autant de réponses pratiques à cette contradiction inscrite au principe même de l'émission littéraire.

Par delà la variété des émissions littéraires qui se sont succédé ou qui coexistent, on peut dégager, nous semble-t-il, des types bien distincts de définitions implicites de la lecture, emblématisées chacune dans une émission de ce point de vue exemplaire.

\section{Le livre dans les émissions littéraires}

\section{Lectures pour tous : l'instruction d'un dossier}

17 A bien des égards, Lectures pour tous est une émission remarquable, tenue aujourd'hui encore pour un modèle du genre. C'est d'abord la plus ancienne des émissions littéraires (et la seule, de 1953 à 1960, date de création de la série Portrait souvenir de R. Stéphane), celle aussi dont la longévité fut la plus grande (280 émissions de 1953 à 1968) ${ }^{13}$. C'est enfin, et surtout, pour ce qui nous concerne, celle qui a d'emblée posé l'entretien avec l'auteur comme genre canonique de convocation du livre à la télévision. La sollicitation de la parole publique de l'écrivain, chose alors insolite, présuppose que lui ait été reconnu un certain horizon de pertinence, celui-là même que nous visons à déterminer, prenant appui pour cela sur les propos de Pierre Dumayet lui-même, et sur la transcription de deux entretiens.

\section{Écrire et vouloir dire}

Si le livre est une œuvre de fiction, le problème est de trouver les questions qui en même temps éveillent et satisfassent la curiosité du public ; satisfaire et éveiller, car le public n'a aucune raison de s'intéresser à l'aventure de personnages imaginaires qu'il ne connaît pas. Examinons de plus près les rapports de l'interviewer et de l'auteur d'une œuvre de fiction. Pour l'auteur son livre est une chose faite, il aime en entendre parler mais il n'aime pas tellement en parler. De plus, il a écrit son livre comme si aucune question ne devait lui être posée à ce propos, comme s'il n'avait pas, en quelque sorte, à en répondre. S'il vient à la télévision, c'est parce qu'il pense que cela aidera son livre, c'est pour son livre. L'idéal serait d'interviewer 
les livres; et la première partie de l'entretien qu'on a avec un écrivain consiste justement, le plus souvent, à lui expliquer qu'on ne peut pas interviewer le livre, car l'interview est le contraire du compte rendu. On doit donc interroger l'auteur comme si son livre n'était pas une chose faite, mais un projet, on lui demande ce qu'il a voulu faire alors qu'il l'a déjà fait. Ce n'est donc pas le romancier présent à qui nous nous adressons, mais l'auteur passé. On retrouve ici la forme de contemporanéité que je signalais tout à l'heure. Nous, nous avons lu le livre alors que l'auteur, lui l'a écrit et, bizarrement, nous nous trouvons dans une situation privilégiée par rapport à lui. Car toutes les phrases d'un livre sont des questions virtuelles que l'on peut poser à son auteur. On a donc lu le livre et on a l'auteur devant soi : que faire ? Quelquefois on ne trouve rien à faire, et rien n'est plus triste que, après avoir lu un livre, de n'avoir vraiment aucune question à poser à son auteur ; heureusement c'est rare. La plupart du temps on tente de confronter telle phrase ou tel personnage avec l'auteur; en lisant le livre, on l'espionne, on recherche cette liaison. Ainsi il m'est arrivé une fois d'être content en lisant un roman de Jacques de Bour-bon-Busset. Je crois qu'il s'agissait d'Antoine, en tout cas d'un livre dont le héros est un personnage masculin; au détour d'une phrase, j'ai découvert un participe passé, qui se rapportait à ce héros, et qui était écrit au féminin. J'ai montré cette faute à l'auteur qui a rougi et qui m'a dit alors que ce héros masculin n'était en fait que la transposition d'un personnage féminin réel. J'ai estimé alors que j'avais bien lu ce livre ${ }^{14}$. qu'il a écrites, Pierre Dumayet interrogeant François Mauriac ne fait rien d'autre ${ }^{15}$.

P. D. - Maître, ces mémoires ne sont pas un livre de souvenirs, n'est-ce pas?

F. M. - On m'a souvent demandé d'écrire mes souvenirs, j'y ai toujours répugné, non pas tellement à cause de moi-même ; prenez par exemple Si le grain ne meurt de Gide, vous retrouvez là son père, sa mère, tous les êtres de son enfance. J'avoue que j'ai toujours reculé devant cette résurrection forcée que l'on impose aux pauvres morts de sa famille. Qu'ils reposent en paix! Alors ne voulant pas parler des miens et ne voulant parler que de moi-même, j'ai eu l'idée de chercher mon reflet dans les lectures de ma vie... J'ai dit les lectures de ma vie, non, je me trompais ; ce n'est qu'une petite partie des lectures de ma vie mais enfin, ce sont, si vous voulez celles qui ont le plus compté pour moi.

P. D. - Vous écrivez " c'est à mon âge qu'hérédité devient autre chose qu'un mot ». Quel a été le rôle de vos parents, de vos ancêtres dans votre vie de créateur?

F. M. - Oh, elle a été très grande. Je crois que j'ai dit, si mes souvenirs ne me trompent pas: en me demandant si la vie que j'ai eue, enfin cette vie, comment dirais-je, tellement réussie au point de vue du monde mais qui a commencé par être la vie d'un poète, car au départ j'étais un poète, et quand je dis un poète, ce n'est pas parce que j'avais publié des volumes de vers mais vraiment parce que j'avais dès l'enfance et dès l'adolescence une conception poétique de la vie; si vous voulez, un goût de transformer la vie qui aurait pu m'entraîner bien loin et surtout, qui aurait pu m'entraîner sur les routes de la malédiction; et moi, j'ai abouti à l'Académie française, au prix Nobel alors que Nerval a abouti au bout d'une corde, alors que le pauvre Verlaine est mort dans une chambre de prostituée, sur une natte. Alors au fond, quand je pense à mes ancêtres, je crois que c'est à ce propos que je disais la phrase que vous avez citée, c'est que je crois que les miens, la bourgeoisie dont je suis sorti a agi sur moi comme des freins puissants, vous comprenez? Les morts, dans une destinée comme la mienne, jouent des rôles de freins, voilà ce que j'ai voulu dire.

P. D. - Non seulement vos ancêtres vous ont empêché de rester un poète seulement, mais ils vous ont aussi entraîné à devenir un romancier, dites-vous?

F. M. - Oui, je vois la phrase à laquelle vous faites allusion, et là, voyez-vous, les critiques, je ne veux pas dire ont été plus que gentils et ne m'ont fait que des compliments, mais moi, je serais tenté de faire mon autocritique. En relisant ce 
livre, une des choses que je me reproche, c'est d'avoir dit de temps en temps des choses qui ne me paraissent pas très vraies; que j'ai écrites naturellement en toute bonne foi ; mais sans m'en rendre compte, je crois que j'ai cédé un peu à la recherche de l'effet. Et, en particulier, quand je raconte que les miens m'ayant en quelque sorte détourné d'être vraiment un poète, je me suis payé sur la dette et que j'ai écrit des romans sur la bourgeoisie française. Eh bien c'est ingénieux, je crois que c'est plus ingénieux que vrai; à la réflexion, j'ai écrit des romans sur la bourgeoisie parce que j'ai décrit le milieu que je connaissais parce que je l'observais naturellement autour de moi, mais aussi parce que je le portais au dedans de moi.

P. D. - Vous écrivez : « Nul ne se sera tenu plus obstinément que moi à l'arrièregarde des lettres ». Comment devons-nous comprendre cela?

F. M - Ah ! Voilà que vous me prenez encore en flagrant délit de la recherche de l'effet ! Voilà, tenez, le type de la phrase : «Je me suis tenu obstinément à l'arrièregarde des lettres...», ce n'est pas absolument faux... Tenez, cela signifie par exemple qu'en 1912, quand j'étais l'ami de Cocteau... Je suis son aîné d'un tout petit peu mais nous sommes partis ensemble, nous étions copains. Et puis, un beau jour, j'ai vu Cocteau monter dans le train de Diaghilev, des ballets russes, de Picasso ; je l'ai vu partir, et moi j'avais nettement l'impression de rester sur le quai. Mais pas du tout, comment dirais-je, avec un sentiment de frustration parce que je voyais, comment dirais-je, partir Cocteau vers l'avant-garde. Moi, j'avais le sentiment que ce que j'avais à dire, si j'avais quelque chose à dire, n'était pas à l'avant-garde mais plutôt dans mon passé ou au dedans de moi, si vous préférez. Mais que mon affaire à moi, si vous voulez, ce n'était pas Picasso, si vous voulez.

P. D. - Je voudrais vous poser une toute dernière question, maitre. Des livres que vous aimez, vous dites qu'ils sont " habitables", voulez-vous me dire ce que vous entendez par là?

F. M. - Ça a pour moi une très grande signification. Je me demande très souvent si les livres que mes cadets aiment, je pense à l'Ulysse de Joyce, si l'ayant lu, ils y reviennent souvent. J'appelle une œuvre habitable une œuvre comme celle de Balzac, de Dickens, Proust, dans laquelle j'entre, je sors. Je suis septuagénaire, et bien je peux dire que depuis cinquante ans j'habite Balzac, j'habite Dickens, Dostoïevski, Tolstoï. Moi, je prends Balzac, mais à chaque instant ! Je prends Proust à chaque instant ! Bon, il y a des livres que mes cadets aiment et que j'aime aussi, mais par exemple j'aime profondément Kafka, ses livres, je les ai lus une fois, mais plutôt crever que d'y rentrer. Je n'ai jamais envie de recommencer un cauchemar [gros plan sur P. D. qui sourit.].

P. D. - Merci. et quasi obligée au texte (vous écrivez..., vous dites...) vient légitimer le questionnement même, réaffirmant à chaque reprise une déférence marquée d'entrée (Maître...). C'est l'auteur, dont la présence sur le plateau est alors proprement inédite, qui est ici la figure centrale, et non pas le livre, lequel fournit simplement un tissu de questions (im)pertinentes à lui retourner. En devant répondre de son texte, en devant faire face par exemple à un "flagrant délit de recherche de l'effet", l'auteur est supposé se révéler à nous. Ce qui se déroule ainsi sous les yeux des téléspectateurs, c'est finalement une sorte d'examen de conscience de l'auteur, l'usage répété du gros plan visant à en saisir la trace sur son visage.

Je trouve que ce qu'il y a de plus intéressant à la télévision, c'est de voir quelqu'un chercher plutôt que trouver. A partir du moment où on préfère le paysage de la recherche, le paysage de la gueule de quelqu'un qui recherche, à celle de quelqu'un qui a déjà la réponse et qui vous la ressort, je crois que l'on préfère être en direct et assister non pas à un débat mais à un entretien. Le débat de Pivot, c'est très bien mais c'est quelque chose qui concerne les idées que sont censés avoir les gens qui sont là. C'est un 
combat d'idées et ce n'est pas du tout la même chose que d'essayer de montrer, de révéler une personne par l'intermédiaire à la fois des caméras (qui ne sont pas de lui) et d'un livre (qui est le sien). C'est comme ça que je vois la chose. Un écrivain, c'est quelqu'un sur lequel on a un dossier fourni par lui-même. Cela a donc un côté un peu caméra invisible puisque l'auteur ne sait pas les phrases de son bouquin sur lesquelles on a l'intention de lui poser des questions. Sa position est d'incertitude. Tandis que s'il s'agit de dire ce qu'il pense, il sait ce qu'il pense, par définition [.... ${ }^{16}$.

\section{L'auteur responsable de son œuvre}

Dans Lectures pour tous il y avait l'écrivain en présence des caméras et son livre était plutôt considéré comme un dossier. Quelles étaient les questions intéressantes que l'on pouvait poser à un monsieur qui était là après avoir lu un livre dont il était non seulement l'auteur mais aussi le responsable. C'était plutôt la confrontation, en présence de caméras, d'un monsieur et de son œuvre ${ }^{17}$.

Après le générique de l'émission, gros plan sur une couverture NRF : Louis-Ferdinand Céline, D'un château l'autre.

P. D. - Vous avez lu le titre de ce livre : D'un château l'autre, vous avez lu aussi le nom de l'auteur : Louis-Ferdinand Céline. Monsieur Céline, avant d'entrer dans ce livre, je voudrais vous poser une question à propos de vos livres précédents; question qui est impliquée par celui-ci. Il semble que vous soyez maintenant surpris de cette avalanche de catastrophes qui ont été la conséquence de vos livres, particulièrement du Voyage. Vous dites que c'est du Voyage au bout de la nuit que sont nés tous vos ennuis, ennui étant ici un mot très faible. Vous ne vous attendiez pas... L.-F. C. - C'est-à-dire que je suis parti dans l'écriture des livres sans vouloir obtenir une notoriété quelconque ; je pensais simplement en tirer un honnête bénéfice pour me payer un petit appartement dont $\mathrm{j}$ 'avais bien besoin à l'époque. Et puis les choses se sont développées de telle sorte que la vie d'un honnête médecin est devenue impossible, jusqu'au moment où je me suis permis de m'occuper de politique et ce fut évidemment le signal d'une ruée, d'un hallali qui me poursuit encore. Voilà.

P. D. - Oui mais ce qui m'intéresse, et c'est la raison pour laquelle je vous pose la question, c'est ceci : est-ce qu'en écrivant le Voyage au bout de la nuit par exemple, et c'est cette phrase de D'un château l'autre qui me le fait croire, vous avez pu croire écrire ce livre impunément presque ; enfin, sans penser aux conséquences?

L.-F. C. - Absolument sans conséquences! Je croyais qu'on y prendrait un petit intérêt comme à lire une nouvelle, et puis que, ayant vendu suffisamment de ce petit livre, je retournerais à ma médecine tranquillement, avec un appartement dont je n'aurais plus la hantise de payer le terme. Parce qu'à ce moment là on avait la hantise du terme, on ne l'a plus. J'ai dit: ce sera une hantise de moins. Voilà, c'était pour ma tranquillité que j'ai écrit le Voyage au bout de la nuit.

P. D. - A aucun moment vous ne croyiez à votre violence, vous ne la conceviez pas?

L.-F. C. - Je ne me vois pas violent du tout; je n'ai jamais été violent. J'ai toujours soigné avec beaucoup de douceur, si j'ose dire. J'ai sauvé énormément de gens, d'animaux. J'ai vécu dans la violence, mais moi-même je ne l'étais absolument pas. Et les livres, très franchement, que j'ai pu écrire, étaient faits justement contre la violence. Je sentais une guerre venir et je dénonçais les motifs de la guerre. L'histoire m'a donné raison... pas les hommes.

P. D. - Je voudrais que nous entrions tout de même quelques instants dans votre livre.

L.-F. C. - Monsieur, je vous en prie.

P. D. - Dans celui-ci : D'un château l'autre. Dites quels sont ces châteaux?

L.-E C. - Figurez-vous que je me suis arrêté là, j'en ai bien d'autres à raconter des 
châteaux, par exemple celui des Ho-henzollern est assez notable, c'est le lit de la dynastie mère de l'Europe ; les châteaux qu'il a fallu que je connaisse aussi. Et si la vie me permet de continuer mes petits scribouillages, je pourrai conduire les personnes qui voudront bien venir jusque-là vers d'autres châteaux où il s'est passé d'autres choses. C'est ce qui représente aussi un intérêt touristique. Parce qu'on m'a reproché beaucoup de danser dans une assiette; alors j'ai dit maintenant je vais promener les lecteurs, parce que peut-être ça leur plairait mieux. Comme on n'a pas beaucoup vendu de livres depuis que je suis rentré, je me dis peut-être c'est parce que je les promène dans une assiette; bien maintemant, je vais leur faire faire du tourisme ; alors j'ai entrepris le tourisme. Alors, s'ils veulent bien me suivre, j'en ai bien d'autres châteaux, et puis des beaux, où j'ai vécu : à Rosenburg, à Copenhague. D'autres châteaux... et puis des chaumières.

P. D. - Mais tout de même, dans D'un château l'autre, il est beaucoup plus question de Sigmaringen que de...

L.-F. C. - Oh ! je n'ai pas encore eu le temps. Je suis arrivé à 400 pages à ce moment là, c'était le moment de livrer les choses à Monsieur mon vénéré ami éditeur Gallimard, et évidemment que j'ai coupé court.

P. D. - Mais il est tout de même déjà assez question dans ce livre de Sigmaringen ; enfin, c'est une question que vous privilégiez.

L.-F. C. - Je la quitterai n'est-ce pas, parce qu'on ne peut pas lasser l'attention de ceux qui veulent bien...

P. D. - Mais dites-moi pourquoi, dites-nous pourquoi plutôt, ce château-là vous intéressait particulièrement à décrire?

L.-F. C. - Parce qu'il était curieux, vu que j'imaginais très bien la vie du Moyen Age : les seigneurs chez eux et les vilains autour.

P. D. - Oui. Vous ne voulez pas me dire en somme pourquoi ce château vous intéressait, pourquoi vous avez eu envie d'en parler.

L.-F. C - Il est intéressant en ce qu'il est assez cinéma n'est-ce pas, et puis il est pittoresque, il existe historiquement. Disons que c'est un moment d'histoire de France n'est-ce pas, l'affaire Pétain. Pétain, Laval, etc. je ne les nomme pas tous.

[...]

P. D. - Je veux vous poser une dernière question, plus exactement, vous demander un dernier mot, la mode est au mot de la fin en ce moment. Vous m'avez tout à l'heure, mes dernières paroles, si j'en avais, ce serait...

L.-F. C. - Ben voilà, je vois dans ces flots d'invectives, des gens qui boivent, qui mangent, qui dorment, qui font toutes les fonctions humaines qui sont toutes assez vulgaires et je dirais qu'ils sont lourds, que leur esprit est lourd; il n'a jamais cessé d'être lourd... Il y a très peu de légèreté chez l'homme, et maintenant, il est extraordinaire de lourdeur, il est extraordinairement lourd. Nous aurons peut-être un jour une révolte de l'esprit contre le poids, mais ce n'est pas pour demain. On est lourd! Alors, si j'avais à mourir, j'ai dit : ils étaient lourds ! S'ils étaient méchants, c'est parce qu'ils étaient lourds. Ils pèsent, ils sont infirmes; l'infirmité les rend lourds ; par conséquent, il faut se méfier, la lourdeur les rend prêts à tout... C'est effrayant.

P. D. - Merci.

23 A peine l'auteur est-il apparu en personne sur la scène publique qu'il est sommé de répondre non seulement de son texte, mais de son œuvre, de son passage à l'acte d'écrire ; car on ne saurait écrire impunément. Ce n'est plus uniquement la lecture du texte mais de l'œuvre qui s'opère par la personne d'un écrivain dont la parole devient autojustificatrice. Lectures pour tous le manifeste d'emblée : l'écrivain en tant que tel, c'est-à-dire en sa qualité même d'écrivain, s'expose, dans sa confrontation, sur le plateau, avec son texte ou son œuvre. Au point pour Céline, par la trivialité des motifs invoqués à l'écriture, de plaider l'irresponsabilité, de se dénier écrivain et ne prétendre être que simple auteur de «scribouillage »! 
Ici, il faut considérer à nouveau le type de questionnement adressé à l'auteur, et la position paradoxale, impliquée par le dispositif même de l'entretien, que celui-ci occupe. Les questions ne sont autres que des phrases de son livre retournées à l'auteur. L'omniprésence de la référence textuelle, qui légitime le questionnement, présuppose une lecture préalable du livre par le questionneur, et en manifeste le caractère paradoxal. Il s'agit d'une lecture qui n'est pas réception, elle consiste simplement à prendre acte de la chose écrite, indépendamment de toute espèce d'interprétation. Un tel questionnement institue proprement l'auteur en récepteur de son propre texte, de sa propre œuvre. En ce sens, Lectures pour tous est pour ainsi dire le degré zéro de la figuration télévisée de la lecture, par cette identification de la personne du lecteur à celle de l'écrivain, dont le questionneur n'est que l'opérateur. Elle met en scène en d'autres termes une sorte de "scène primitive " d'avant la socialisation, où écriture et lecture ne font qu'un dans la conscience du créateur (c'est en ce sens qu'on a pu dire qu'il procédait alors à son " examen de conscience "). Les téléspectateurs, quant à eux, sont conviés à être les témoins privilégiés de cette lecture singulière, de cette relation intime ${ }^{19}$. Ils sont eux-mêmes constitués en public élu, auquel une lecture ainsi figurée ne peut manquer d'apparaitre comme une expérience cultivée, lettrée, consistant à rentrer dans les raisons de l'écriture, qui ne sont autres que celles de l'auteur.

\section{Lire c'est vivre : quand le « monde du texte » rencontre le « monde du lecteur »}

Exception faite de quelques émissions de l'après-midi (Aujourd'hui madame, de 1975 à 1981, puis Aujourd'hui la vie, de 1982 à 1986, qui proposent deux fois par mois un débat en direct entre des auteurs et leurs lectrices), Lire c'est vivre (de 1975 à 1987) est la seule véritable tentative de présentation du livre du point de vue de sa lecture ordinaire. Pierre Dumayet confie ici le livre à des lecteurs privilégiés (des ouvriers parisiens pour L'Assommoir de Zola, d'anciens prisonniers des camps pour Souvenirs de la maison des morts de Dostoïevski...), puis les interroge, à partir des passages qu'ils y ont soulignés, non pas tant sur le contenu littéraire de l'œuvre que sur les rapports qu'elle entretient avec leur propre expérience. Entre les séquences d'entretien, filmées dans le cadre familier des lecteurs, et pendant que sont diffusés à l'écran des extraits de films ou de documents d'archives en relation avec l'œuvre évoquée, Pierre Dumayet, dans le rôle du récitant, raconte le livre en voix off, par fragments successifs, qui conduisent à chaque fois à un épisode jugé significatif et souligné par les lecteurs ; dans la continuité de la voix off, la lecture du passage ou simplement de la phrase en question succède alors au récit... et voici que Pierre Dumayet, le livre à la main, interroge le lecteur. C'est cette alternance entre le monde du texte mis en récit et le monde du lecteur manifesté par l'entretien, que nous voudrions restituer dans cette transcription d'un Lire c'est vivre consacré à la lecture de Germinal (Zola) en pays minier cévenol ${ }^{20}$.

Avant d'entrer dans le livre, Pierre Dumayet nous présente ceux qui « ont lu Germinal un crayon à la main »; présentation ponctuée d'extraits d'entretiens significatifs.

Un ancien mineur racontant l'accident du travail qui l'a amputé d'un pied à 18 ans.

Un autre, M. Morin, nous prenant à témoin :

- Je vais vous dire quelque chose que vous ne croirez pas: une année, j’ai fait 399 journées payées.

$\mathrm{M}^{\mathrm{me}}$ Michel, militante CGT, veuve de mineur, répondant catégoriquement : 
P. D. - Si vous aviez à juger Soubarine (le militant qui sabote le puits à la fin de Germinal)?

$\mathrm{M}^{\mathrm{me}}$ M. - Moi, je le pendrais !... Soubarine, pour moi, c'est quelque chose de très dur.

R. Amouroux, fils de mineur, ancien métallo entré à la mine comme tant d'autres à la suite d'une mobilisation collective pour garder ouverte la dernière mine de la région, expliquant les raisons de son choix :

P. D. - Si vous aviez lu Germinal juste avant de faire votre demande pour être intégré à la mine, vous l'auriez faite votre demande?

R. A. - Ben... je sais pas. Je comprends que celui qui lit Germinal avant de faire sa demande ne la fasse pas parce qu'il se dit: "C'est pas possible, on travaille encore comme ça ", ce sont des conditions de travail qui ne peuvent être admises par personne. S'il y en a qui ont encore des doutes, je peux leur dire : c'est fini ça.

Enfin, le vieux Démosthène expliquant pourquoi il n'a rien souligné dans le livre :

- D'habitude, je ne souligne pas dans un livre. D'habitude, je me rappelle avec ma mémoire.

Pierre Dumayet raconte le début du livre (mise en place des lieux, des personnages). Le voici maintenant avec $\mathrm{M}^{\text {me }}$ Michel, en train de lui lire à voix haute un passage (c'est « la Maheut » qui explique pourquoi elle a sept enfants).

P. D. - ... « Et puis, quand ça grandissait...»

M. M. - « ça rapportait » (reprend la femme, de mémoire, devançant la lecture) : ça, ça m'a choquée. Ça dénote un caractère que nous n'avons plus, parce que nous, nous vivons pour nos enfants alors que, d'après la manière dont c'est écrit, il semblerait que les enfants aidaient à faire vivre les parents.

P. D. - C'est aussi ce qui explique que toutes les familles sont furieuses quand quelqu'un veut se marier jeune.

M. M. - Cette colère après Zacharie (parce qu'il a fait deux enfants)! Alors il faut pas qu'il se marie parce qu'il partirait de la maison et on n'aurait plus son salaire. Ça semble pas vrai!

Pierre Dumayet reprend en voix off: choqué, Démosthène ne l'est pas, il semble avoir connu ça dans l'ancien temps.

D. - (raconte comment ça se passait avec les enfants : c'était chose courante qu'un jeune reste jusqu'à 20 ans à la maison)... voilà le parallèle que je fais avec machin.

P. D. - Pour les raisons que dit Zola aussi ; pour garder la paye à la maison?

D. - Voilà (continue à raconter comment ça se passait).

Pierre Dumayet continue le récit jusqu'à l'épisode de l'arrivée de Lantier, à la recherche d'embauche : «Du travail ? Non, il ne sait pas s'il y en a le grand-père Bonnemore. Le grand-père Bonnemore s'est présenté ainsi à Lantier: cinquante ans de mine dont quarante-cinq au fond, c'est joli, hein!»

P. D. - Vous avez souligné ça.

R. A. - Quarante-cinq ans de mine au fond ! Je voudrais pas le faire hein! (en riant).

Nous, on réclame les trente ans de service. Eux, ils avaient pas de problèmes de retraite ni rien. Ils venaient au monde et ils mouraient à la mine. C'est pas normal.

Pierre Dumayet reprend en voix off: à M. Corbier (infirmier dont le père avait quitté la mine pour les champs), la phrase paraît naturelle.

M. C. - On retrouve ça chez nous : un type est fier de dire ça, et moi qui n'y ait jamais travaillé je le plains, je trouve pas ça « joli». Et ça, on le retrouve toujours, cette fierté d'avoir travaillé longtemps dans de mauvaises conditions (il pointe l'index sur le livre que P. D. tient ouvert près de lui).

P. D. - (lit plusieurs extraits). C'est vrai qu'il y a un peu une vénération de la mine quand même et du travail dur.

M. C. - Oui, chez nous on disait : le mineur est un seigneur. 
Pierre Dumayet continue (Lantier est embauché et pris en pension dans une famille de mineurs) : ça se faisait ici aussi autrefois, M. Morin s'en souvient :

M. M. - Dans les familles de mineurs, on prenait des pensionnaires et ça pouvait finir par des mariages ; mais il n'y avait pas tout de même cette promiscuité que j'ai lue dans ce livre. Ça me semble un peu quand même exagéré par rapport à ce que j'ai connu.

Pierre Dumayet raconte la sexualité débordante, les jours de disette, et enfin la grève : c'était risqué parce qu'on risquait de perdre son livret. Ici, reprend Pierre Dumayet, il n'y avait pas de livret mais le registre. $\mathrm{M}^{\mathrm{me}}$ Michel en a retrouvé un (gros plan sur une page avec une inscription à l'encre rouge : « fut un des chefs de la grève »).

Les anciens se souviennent : « Nous aussi, on en a fait des grèves !»; et ils racontent comment ceux qui étaient marqués à l'encre rouge ont dû quitter la région et sont partis parfois jusqu'en Amérique.

La grève se durcit et c'est l'épisode de la mise à mort du contremaître châtré par les femmes.

$\mathrm{M}^{\mathrm{me}}$ Michel - ... Enfin, il est mort tout seul; il est tombé du toit... Mais enfin, j'ai peine à y croire. C'est du roman, c'est du roman !... Moi, ici, j'ai vu le directeur qui voulait pas sortir sous la pluie, sorti à coup de fesses par une femme.

Les grévistes sont maintenant aux abois et Lantier est accusé par ses propres camarades de les avoir entraînés à la grève.

$\mathrm{M}^{\mathrm{me}}$ Michel - Lantier, je le plains, parce qu'il était pas fait pour être mineur ; c'est un métier qu'on se lègue. Je le plains, à la fin, quand il est rejeté.

R. Amouroux - Lantier, je crois qu'on lui doit quelque chose quand même, nous. C'est à nos ancêtres qu'on doit que la mine ne soit plus ce qu'elle était.

Et voici le dénouement: Soubarine, militant anarchiste, a saboté le puits de mine. Lantier survit à l'explosion, mais reste plusieurs jours bloqué au fond.

P. D. - Il y a deux minutes, avant que la caméra ne tourne, vous m'avez laissé entendre que vous aviez souligné des passages mais que, finalement, ça n'est pas exactement ce que vous pensez, au fond du cœur, de Germinal.

Mme Michel - J'avais lu Germinal il y a quelques années ; ça m'avait énormément plu ; et maintenant, en le relisant pour vous, j'ai trouvé des choses différentes. Il y a deux parties : la vie des mineurs, la vraie, la vérité; et puis un roman, difficile à suivre parce qu'il est un peu irréel. Je peux me tromper, mais j'ai été déçue. La fin, par exemple, c'est invraisemblable. Qu'on puisse vivre pendant neuf jours, je crois, dans l'eau, avec un peu de grisou, sans manger et sans boire, sans lumière. C'est pas faisable, ça!

41 De la même manière que l'écrivain, sur le plateau de Lectures pour tous, devait assumer la responsabilité de son écriture, c'est au tour du lecteur de répondre de sa lecture. Il doit pareillement faire face aux phrases que lui retourne Pierre Dumayet, à cette différence près qu'il les a ici préalablement choisies objectivant ainsi sa propre activité liseuse. Or, que disent ces lecteurs interrogés sur leurs lectures? Ils parlent de leur vie, de ce qu'ils ont vécu. Davantage que les lecteurs du livre, ils en sont proprement des personnages; tandis que les personnages du roman sortent du livre pour devenir leurs semblables, honnis ou respectés («Soubarine, je le pendrais!", "Je crois qu'on lui [Lantier] doit quelque chose quand même, nous »). La confusion est d'ailleurs entretenue par le dispositif même de l'émission, alternant séquences de récit et d'entretien, qui a pour effet d'inscrire les souvenirs de lecteurs dans le déroulement narratif de l'intrigue. Le livre intègre alors l'expérience vécue de ses lecteurs: le 
Germinal présenté aux téléspectateurs excède sa textualité, augmenté par sa propre lecture.

Une fois encore la présentation télévisée de l'œuvre opère la fusion artificielle de l'écriture et de la lecture, celle-ci ne se tient plus cependant dans la conscience de l'auteur (comme si le livre n'était pas encore écrit, comme s'il n'était encore que projet), mais dans le livre. Un livre démultiplié de fait en autant d'exemplaires soulignés qu'il y a de lecteurs, et dont l'unicité est fictivement rétablie dès lors que Pierre Dumayet en est le dépositaire exclusif. C'est lui qui raconte et lit en voix off. C'est toujours lui qui interroge les lecteurs, le livre à la main, ouvert à la page soulignée. De cette manière, dans l'alternance entre séquence de récit et séquence d'entretien, Pierre Dumayet opère à notre insu un recouvrement du livre-texte par le livre-objet physique lu et souligné ; mieux, il les identifie proprement. Qu'en est-il alors de ces lecteurs d'occasion, commis à la lecture et aussitôt dessaisis du livre qu'ils ont pourtant marqué de l'empreinte de leur lecture ? Tout ne se passe-t-il pas en fait comme s'ils n'en étaient pas propriétaires, mais qu'on le leur avait laissé en prêt le temps de la lecture ? Ils sont simplement convoqués pour attester de la lisibilité de l'œuvre, dont ils apportent la preuve en marquant ce qui les a marqués. C'est dire que leur lecture est une lecture instrumentée, ce qu'implique d'ailleurs le principe même de l'émission. On présuppose en effet que, de par leur origine, les lecteurs choisis auront quelque chose à dire à propos d'un livre qui n'aura pas manqué de « leur parler ». Suscitée aux fins pratiques de l'émission, leur lecture (première lecture ou relecture) est en retour tenue par un tel a priori: aussi n'est-il question que de vérité référentielle alors que c'est d'un roman dont il s'agit. Dans le cas d'une relecture, la contrainte exercée par le cadre est manifeste : il y a quelques années, le roman avait plu, aujourd'hui au contraire on est déçu par tant d'invraisemblances! On peut, dans ces conditions, se demander ce qu'auraient pu dire de Germinal des lecteurs n'ayant rien à voir avec la mine. Tel est le paradoxe d'une émission qui entend établir que le monde du livre n'est pas coupé du monde de la vie, que Lire, c'est vivre, et la lecture par conséquent, ne se réduisent pas à une expérience cultivée. De fait, l'association d'un lectorat spécifique à chacun des livres présentés ainsi que la mise entre parenthèses de la dimension proprement littéraire de l'œuvre ne sont que les conditions implicites nécessaires pour assurer à chaque reprise une rencontre exemplaire entre les mondes du texte et du lecteur. En ce sens, Lire c' est vivre opère un véritable tour de force en figurant une lecture entendue comme expérience intime, en nous faisant assister à une expérience ô combien singulière : le lecteur qui se retrouve dans le livre. Quant au téléspectateur, il ne tient qu'à lui de trouver à son tour le livre de sa vie, de « trouver sa vie » dans un livre.

\section{Libre et change : la lecture en débat}

43 Avec Libre et change, et également Apostrophes et Ex-libris que nous considérerons par la suite, nous abordons les émissions littéraires d'aujourd'hui, contemporaines - du moins - de notre étude. Si nous retenons ici l'émission de Michel Polac, en dépit de sa brève durée d'existence (novembre 1987-juin 1989), c'est qu'elle objective pareillement un type singulier de représentation de la lecture, déjà partiellement à l'œuvre, il est vrai, dans la précédente émission du même Michel Polac Droit de réponse (décembre 1981juillet 1987),-laquelle traitait tant de l'actualité littéraire que des dossiers brûlants de l'actualité sociale et politique. Pour autant qu'il s'agisse cette fois d'une émission 
spécifiquement littéraire, Libre et change n'en conserve pas moins certaines des caractéristiques formelles de sa devancière.

La formule du débat en direct tout d'abord, mêlant auteurs et critiques littéraires, ou réunissant pour une revue de presse périodique, le même cercle de journalistes. L'engagement de Michel Polac ensuite; non seulement susceptible à tout moment de prendre ouvertement parti, mais dont les prises de position constituent le plus souvent l'amorce du débat. Une organisation du plateau enfin, qui accentue les situations de visà-vis, de face à face, de côte à côte, de dos à dos : à l'extrémité d'une table basse se trouve Michel Polac, de part et d'autre les participants se tiennent sur deux rangées, la seconde légèrement surélevée. Ainsi agencé, cet espace du déploiement de la parole est sans cesse reconfiguré par une caméra mobile qui délaisse le champ-contre champ des débats pré-réglés pour multiplier les contre-plongées et les effets de profondeur de champ... comme si c'était le débat, le véritable sujet de l'émission.

Tels sont les principes formels de Libre et change à partir desquels est mise en scène la confrontation des réceptions, lesquelles dépassent dès lors le strict rapport intime au texte pour devenir choses publiques et débattables. C'est précisément les modalités et les effets pratiques de la convocation, du compte rendu de l'expérience lectorale, en bref, de sa socialisation, que nous voudrions considérer, à partir de quelques transcriptions de fragments d'émissions.

A propos d'un livre de Vonnegut que le critique R. Sorin vient de présenter :

M. P. - Il faut dire que ce livre, au début, j'y suis rentré un peu difficilement, et à la fois facilement, parce que ça se lit vraiment comme un polar [au premier plan, quelqu'un opine du chef], mais il a un côté glacé, froid, avec des personnages auxquels on n'arrive pas à croire, des silhouettes [gros plan sur R. Sorin : «Ce sont des silhouettes, oui »], des marionnetttes; et peu à peu, on est pris complètement par l'histoire. Au début, bien sûr, on le lit un peu comme une fable, finalement on est complètement captivé [il feuillette le livre machinalement] et on est pris comme par un vrai roman à la fin. D'ailleurs, les personnages sont plus fouillés.

[Il lit l'introduction]... Ensuite on lit le livre et on se dit que la morale de l'histoire est absolument à l'opposé de ce qu'il nous dit au début [très gros plan sur son index pointé sur l'introduction). Bon, on peut dévoiler en tout cas le début.

Il commence à raconter l'intrigue en regardant les autres, avec des coups d'œil répétés à R. Sorin, pour approbation. Pendant qu'il dévoile toute l'intrigue, à l'exception de la fin « qui est extraordinaire ", le livre dans ses mains reste ouvert à la première page. Au terme de son récit, Michel Polac cède la parole à R. Jaccard qui le regardait avec insistance, comme s'il avait eu quelque chose à ajouter.

Non, non, simplement ça me fascine la manière dont tu en parles. On a très envie de

le lire.

Là-dessus, Michel Polac repart de plus belle, pour conclure, enthousiaste : "Je crois qu'il faut absolument recommander ce livre ${ }^{21}$ "

Ce dont il est question dans la présentation faite par Michel Polac, ce n'est pas tant du livre que de sa propre lecture, de sa propre réception qu'il tente de faire partager. En sorte que la présentation est proprement indissociable du jugement prescriptif. C'est en effet cette lecture singulière-ci qui anime le livre, qui lui donne vie : le livre en question est d'abord celui dans lequel on rentre difficilement, il est ensuite celui qui captive et nous prend. En d'autres termes, c'est de cette lecture singulière que procède l'envie de lire (ou de ne pas lire) le livre. Le présentateur devient ainsi prescripteur explicite de lecture aussi bien pour les téléspectateurs que pour les participants à l'émission. 
De fait, dès l'émission suivante :

M. P. - J'ai reçu une lettre de Pierre Assouline qui me signale que L'Inondation est très très bien traduit par Barbara Nassarov; et il me dit : "Pour une fois où c'est bien traduit, il faudrait peut-être le signaler.» Il a tout à fait raison. Ce livre que nous vous recommandons maintenant depuis plusieurs semaines est en réédition, et le texte avait été apporté par Barbara Nassarov justement aux éditions Solin qui l'ont édité. Et je dois dire que, à côté d'autres livres très mal traduits, c'est réconfortant de citer celui-là. Parmi les livres très mal traduits et très mal présentés, je vous signale un livre publié aux éditions « Champs » Flammarion, une réédition d'un texte d'Albert Einstein sur sa façon de voir le monde. Le titre, c'est... Non, pas Ce que je crois, mais c'est quelque chose comme ça... Comment je vois le monde. Et c'est un livre tellement mal présenté qu'on commence à le lire en se disant c'est curieux, les chapitres s'enchaînent bizarrement. Et, arrivé, à la page vingt et une, on comprend que ce sont des textes complètement indépendants les uns des autres. Mais, il n'y a rien dans le livre pour le dire et on ne sait même pas quand ça a été publié.

[...]

M. P. - Un petit mot sur Le Voyage à Rome de Moravia?

L. Adler (critique littéraire, habituée de l'émission) - Moi, j'ai trouvé très brouillon, très inodorant. J'ai recommencé à cinq reprises et j'ai trouvé ça mal structuré. C'est toujours les mêmes ingrédients : la sexualité, la jalousie, la recherche du papa... J'ai été extrêmement déçue puisque j'ai été une très grande amoureuse de Moravia. Mais ce dernier texte, je sais pas...

M. P. - Il n'y a pas que ce dernier. Je trouve que ses trois, quatre derniers romans... Moi, j'ai franchement détesté : cet espèce de squelette de roman, froid, terriblement froid ! 23

On l'aura compris, l'engagement de Michel Polac n'est pas une simple option dramaturgique. Il résulte naturellement d'un jugement sans complaisance porté sur le marché de l'édition : la plupart des éditeurs sont des menteurs, la plupart des livres sont très mal traduits, véritable marché de dupes pour les lecteurs. C'est ce qu'il confesse par ailleurs: "Je n'ai plus tellement envie de faire de la télé; si je continue c'est parce que je veux défendre des livres dont on ne parle pas. Aujourd'hui la critique est ciblée, avec quelle complaisance, sur une vingtaine de bouquins. Il faut se battre à mort contre ça. Si je ne parle pas de certains livres, qui le fera ${ }^{24}$ ?» En d'autres termes, la qualité d'un livre n'est pas fonction d'une notoriété injustement discriminatoire ; on ne saurait par conséquent préjuger de ce qui ne tient qu'à la qualité de la réception, seul opérateur de distinction légitime. De là découlent les deux aspects de l'engagement de Michel Polac.

Il s'agit d'une part de considérer des ouvrages qui sortent des sentiers battus de la critique. De type militant, l'engagement déterminé de Michel Polac vise ici à donner leur chance à ces livres fantômes, dont personne ne parle, exclus des circuits du battage médiatique orchestré par la promotion éditoriale et relayé par une critique complaisante. Et ce en les faisant bénéficier à leur tour de la formidable force d'impact de la télévision. A l'effet de prescription implicite résultant d'un tel battage, Michel 
Polac oppose une volonté de prescription, plus encore qu'explicite, revendiquée comme telle, jusque dans ses implications sur les ventes.

Il s'agit d'autre part de rompre le complaisant neutralisme du présentateur d'émissions littéraires, pour faire proprement œuvre de critique, au risque de se couper des éditeurs. «Il y a des tas de gens qui refusent de venir chez moi, pour ne pas rater Apostrophes. C'était déjà comme ça du temps de Droit de réponse alors qu'on faisait vendre autant que Pivot. Mais les éditeurs ne savaient pas si on allait attaquer ou encenser le livre. Ils se disaient : "Chez Pivot, c'est plus sûr." Il faut savoir dire du mal d'un livre, c'est cela qui donne du prix aux compliments ${ }^{25}$. " Savoir dire du mal, telle est la condition nécessaire pour une prescription efficace. Encore faut-il que cela ne résulte pas de la simple expression d'une subjectivité individuelle, mais soit sanctionné par le débat critique.

A l'occasion de la présentation, par Michel Polac, de deux ouvrages, le débat s'engage à propos d'un autre livre celui de Julien Dray, député socialiste présent sur le plateau et entouré de journalistes.

M. P. - Attendez! Attendez, parce qu'on n'a pas encore présenté le livre de Julien Dray ; je voudrais le faire rapidement...

Les participants débattent ensuite de l'ouvrage d'un certain Bensaïd, bien connu de tous et absent du plateau (il s'agit probablement de cette personne que la caméra nous montre à plusieurs reprises faisant la moue ou bien l'air satisfait, dans le public qui se tient sur des gradins installés en retrait du plateau, et dans une semi-obscurité).

M. P. - Je voudrais quand même, si vous le permettez... vous savez que c'est très difficile de critiquer un auteur tout en l'ayant en face de soi. Bon, pour Julien Dray, on s'en est pas mal sortis... et Bensaïd, j'ai appris qu'il était dans la salle, alors je vais quand même lui tendre un micro maintenant qu'on a fini de parler de son livre. Alors, Daniel Bensaïd, s'il veut bien, s'il est d'accord... [celui que nous supposions être Bensaïd sort du public, vient sur le plateau et prend un micro ; il reste debout, les fauteuils étant tous occupés]. On n'a pas d'ailleurs été tellement méchants; je croyais qu'on allait être beaucoup plus méchants. Moi, j'avais des critiques un peu sévères, mais bon...

Bensaïd - Non, c'est pas du tout méchant...

Michel Polac présente, maintenant, un livre de L. Bayer, L'Entreprise France.

M. P. - J'aimerais avoir tout de suite la réaction de Jean-Marcel Bouguereau.

J.-M. B. (L'Evénement du jeudi, ancien de Libération) - Moi, c'est un livre qui m'a énormément énervé. C'est un livre insupportable, énervant, plein d'erreurs, de contre-vérités ; mais à la limite, ce n'est pas ce qui m'a le plus énervé. Ce qui m’a le plus énervé, c'est que ce livre destiné aux Allemands présente la France d'une manière caricaturale, fausse... Bon alors moi, j'adore L. Bayer, je le connais depuis vingt ans, je suis copain avec lui, c'est un type adorable, très gentil, et tout ce que je dis là, je le lui ai dit en face au cours d'un déjeuner. Je ne me serais pas permis sans cela de le dire [pendant tout ce temps, D. Pouchin, livre en main, réclame la parole pour faire une citation; comme il est assis à côté de J.-M. Bouguereau, on le voit sourire en la lisant dans sa tête].

M. P. - Pouchin, la citation!

D. P. (Libération) - Cette phrase est extraordinaire [il commence à la lire], et écoutez bien [il la termine] !

M. P. - Oui, c'est sûr, c'est une des phrases les plus comiques du livre.

57 Une discussion animée s'engage ensuite au cours de laquelle un désaccord oppose le journaliste de Libération à celui du Monde: "Je n'ai jamais dit ça. Ne t'énerve pas 
Dominique, je n'ai jamais dit ça. Nous nous connaissons, tu sais très bien que je n'ai jamais pensé ça ${ }^{26}$ »

Le débat ici introduit n'est pas de ces débats suscités de toutes pièces aux fins pratiques de l'émission, il déborde au contraire d'emblée de son cadre, s'engageant à propos d'un ouvrage non encore présenté. Tout se passe comme si le plateau ne faisait qu'accueillir un débat préalablement amorcé sur d'autres scènes qui en constitueraient la coulisse. En d'autres termes, les différentes parties prenantes d'un tel débat se connaissent par ailleurs : le présentateur, les auteurs, les journalistes ou critiques réunis sur le plateau, voire les auteurs physiquement absents, mais dont le livre est examiné. Tous sont membres de cette communauté intellectuelle qu'ils représentent sur le plateau, et qui est en fait le véritable acteur collectif du débat. Aussi, est-ce à la condition de lui en avoir déjà parlé en particulier que l'on peut effectivement se permettre de «dire du mal » du livre d'un copain sur la scène médiatique ; ce qui apparaît alors non pas tant comme une trahison que comme l'expression d'une intégrité professionnelle qui ne saurait remettre en question la relation interpersonnelle. D'autant que les qualités proprement personnelles de l'auteur, telles sa gentillesse... ne sont pas en cause. Plus largement, l'interconnaissance qui n'est ici l'objet d'aucune dénégation, n'est pas un obstacle à l'exercice d'une critique véritable; elle est à l'opposé un principe de régulation du débat en ce qu'elle implique sinon un accord préalable, du moins un même cadre de réception, de nature à préserver le débat de la vaine polémique. Le plateau de Libre et change est de ce fait le théatre moins de la confrontation que de l'ajustement des points de vue, eu égard notamment à la présence de l'auteur. Comme l'admet Michel Polac, qui pour sa part "avait des critiques un peu sévères ", qu'il n'a finalement pas formulées : contrairement à ce qu'il aurait pu croire, "on n'a pas été tellement méchant ». C'est dire que le débat est une entreprise commune, nécessitant la coopération de chacun, et dont l'issue sanctionne une performance moins individuelle (le brio, la force de conviction de tel ou tel) que collective: "On s'en est pas mal sorti... ». A l'inverse si le procès d'ajustement est menacé par la divergence excessive des points de vue exprimés en direct, sur le plateau, il suffit d'en appeler au principe $a$ priori de la connaissance mutuelle : « Nous nous connaissons, tu sais très bien que je n'ai jamais pensé ça.»

59 Dans cet espace de l'entre-soi qu'est le plateau de Libre et change, les échanges sont libérés de l'impératif de circonspection et de réserve attaché d'ordinaire aux stratégies de la présentation de soi ; ils sont au contraire régis par un principe d'exprimabilité : il n'y a rien qu'on ne puisse, a priori, se dire... pas même reconnaître qu'on n'a pas lu le livre dont il est question.

M. P. - Bartleby l'écrivain? je suis sûr que Dumayet le recommandera chaudement, non?

P. D. - C'est un livre capital quand même.

M. P. - Mais que personne n'a lu. Jacques Thibaud, l'avez-vous lu?

J. T. - Non.

M. P. - Vous voyez, j'étais sûr, c'est pas lu Bartleby! Lisez-le! Je vais vous le donner tout de suite après.

[...]

M. P. - Que pensez-vous du troisième tome des Carnets de Camus?

F. Weyergans - C'est très cher.

M. P. -170 francs, mais ils sont fous !

L. Adler - Le papier est très beau, c'est très bien aéré, il y a une très belle mise en page ; et puis c'est un petit tirage. 
M. P. - Non, Camus n'est pas un petit tirage.

L. A. - Personne ne s'intéressera à ces Carnets, sauf nous, et c'est très intéressant.

M. P. - François, tu l'as lu ce livre?

F. W. - Je l'ai feuilleté, ça se lit pas, parce que c'est... J'ai lu de gauche à droite ${ }^{27}$.

60 A propos des Versets sataniques, le livre de Salman Rushdie, dont Michel Polac, pour lancer la discussion, déplore qu'il fasse la une de l'actualité sans que personne ne l'ait lu:

F.-O. Giesbert (journaliste au Figaro) - On peut dire, entre nous, que le livre de Rushdie n'est pas terrible.

Un confrère - Tu l'as lu?

F.-O. G. - Je ne fais pas partie du petit club des huit personnes qui l'ont lu.

Premier confrère - Alors, pourquoi tu dis : c'est pas terrible?

F.-O. G. - Parce que j'ai essayé de le lire. Je vais pas me farcir un bouquin comme ça $\mathrm{a}^{28}$ !

61 Ne pas avoir lu le livre présenté par Michel Polac, voilà qui peut paraître naturel puisque c'est précisément le propos de l'émission que de donner leur chance aux ouvrages dont on ne parle pas, ou bien encore une façon, ô combien radicale, de «dire du mal » du livre. Dans le cas du livre de Rushdie, c'est d'autre chose qu'il s'agit, comme en témoigne la réaction de Michel Polac :

C'est vrai que celui-là [i.e. Les Versets sataniques] n'est peut-être pas pour nous très facile ; mais les précédents livres de Rushdie, Les Enfants de minuit et La Honte, sont reconnus dans le monde entier comme deux grands livres et c'est un grand écrivain.

Si Michel Polac n'oppose pas cette fois, une injonction à la lecture, à l'argument de l'illisibilité des Versets sataniques, non plus qu'il ne remet en cause son bien fondé, c'est pour la bonne raison qu'un tel argument est ici pour le moins déplacé. C'est l'existence factuelle du livre qui est menacée, par conséquent les questions relatives à la lisibilité du livre, à la qualité de sa réception deviennent secondaires. Il ne s'agit plus simplement de donner sa chance à un ouvrage que personne n'a lu, en discutant de ses qualités propres ; il s'agit de défendre rien moins que le Livre, expression objective de cette liberté fondamentale qu'est la liberté de création, c'est pourquoi il importe autant de rétablir Salman Rushdie en sa qualité de grand écrivain.

En ce sens, " l'affaire Rushdie » est une affaire politique, de la même manière que Libre et change est une émission politique. En l'occurrence, la discussion à propos des Versets sataniques sur le plateau de Libre et change objective ce qui constitue l'arrière-fond de l'émission et qui est pourtant figuré à même son générique dans les images inversées d'un livre en train de brûler. L'entrée en matière de Michel Polac lors de cette même émission est d'ailleurs sans équivoque :

Toujours d'actualité ce générique ! Un livre qui renaît de ses cendres... puisqu'on en

a brûlé tellement, on peut bien les faire renaître.

Le livre, sur le plateau de Libre et change (dont les caractéristiques nous apparaissent dès lors comme étant celles de 1'«Espace public ») n'est pas simplement un bien culturel; il est - plus fondamentalement - une institution emblématique de la démocratie. La lecture pour sa part, pour autant qu'elle invite au débat, renoue avec la fonction politique qui fut proprement la sienne dans «les cercles de la France bourgeoise $^{29} »$. Elle suscite alors cette forme épurée du débat démocratique qu'est la conversation entre pairs, désintéressée, et dont le contenu même (des jugements de goût qui échappent comme tels à la juridiction de la vérité) engage les participants à renoncer à la prétention d'imposer leur point de vue, au profit d'une recherche 
constante de l'ajustement... Encore faut-il que ceux-ci soient véritablement des " hommes de goût ", qu'ils aient, en d'autres termes, l'esprit démocratique.

L'émission vient de commencer, Michel Polac a présenté les participants et il rentre dans le vif du sujet.

M. P. - Alors Guy Hermet, votre livre... Je ne vous connaissais pas, vous êtes professeur à Sciences-po, et je me suis posé des questions en le lisant [il prend le livre et le feuillette], je me suis inquiété. Le Peuple contre la démocratie: est-ce que Monsieur serait un fasciste déguisé (gros plan sur Guy Hermet, tout sourire); et puis, au fil des pages, je me suis dit : non, non. Il y a une ironie profonde [petit rire à l'adresse de Guy Hermet] qui ne peut pas le faire classer dans un camp politique. Et au fond, je ne vous connais pas du tout, je dirais de vous que vous êtes un peu un « anar » de droite.

G. H. - Oui, c'est possible (éclat de rire de Michel Polac) ${ }^{30}$.

\section{Ex-libris : le téléspectateur fait lecteur} existence à la stature médiatique de son promoteur, Patrick Poivre d'Arvor, présentateur vedette du journal télévisé le plus écouté de France ; c'est du moins ce qui ressort de ses propres déclarations.

En 1987, on a pensé à moi pour le journal de 20 heures. A ce moment-là, je pense qu'il était impossible de proposer une émission littéraire. L'objectif numéro un de la chaine restait la rentabilité. Aujourd'hui, avec Ex-libris, j'ai pu proposer ce que j'aime vraiment ${ }^{31}$." "La chaîne a sans doute eu le désir de me faire plaisir: mon journal marche bien et l'on sait que depuis longtemps je rêve d'une émission littéraire ${ }^{32}$.» «Quand j'ai proposé mon idée à P. Le Lay (le directeur de TF1), il m'a immédiatement donné son accord. Il faut dire que ces derniers temps, TF1 avait vraiment besoin de redresser son image ${ }^{33}$.»

On l'aura compris, pour surmonter la sempiternelle antinomie du livre et de la télévision, encore accentuée dès lors que la loi de l'audience remplace le service public, il fallait un homme de télévision qui soit aussi un homme de culture. Cet homme providentiel pariant sur une réconciliation possible de la chaîne commerciale qu'est devenue TF1 avec la culture, c'est Patrick Poivre d'Arvor, dit encore PPDA. D'ailleurs, le sigle bien connu de son nom, il le décline désormais de manière à donner une tonalité littéraire à la bonne image dont il bénéficie déjà auprès du public, en se disant habité «Par la Passion Des Auteurs » (il est lui-même romancier à ses heures). Au demeurant, la personnalisation de l'émission fait écho à cette autre personnalisation qui donne précisément son titre à l'émission : la personnalisation du livre par un ex-libris, petite vignette généralement ornée d'un dessin original que les bibliophiles collaient sur la première page de chacun de leurs volumes. C'est cela même d'ailleurs que figurent les premières images (de synthèse) de l'émission : un fond blanc avec un motif sur lequel viennent se confondre, transparentes, ce qu'on devine être des pages au mouvement qu'elles dessinent à partir d'une reliure fictive qui se tiendrait sur le côté de l'écran. Chacune d'entre elles une fois tournée, imprime sur le fond un élément du générique, de sorte que lorsque le titre paraît, Ex-libris, il constitue comme une légende au motif. Enfin, le fond lui-même qui figurait donc une page blanche, la première page d'un livre, ornée d'un ex-libris, commence d'être tourné, comme par un lecteur qui serait le téléspectateur de l'émission, et dévoile petit à petit le plateau avec PPDA qui nous 
regarde déjà et nous dit : «bonsoir » à peine la première page, complètement tournée, a-t-elle disparu de l'écran. Voilà qui est fait : téléspectateurs il y a quelques instants encore, nous voici devenus lecteurs d'un livre intitulé Ex-libris... A proprement parler, si l'on considère la formule de l'émission («magazine », justement) et sa périodicité, ce serait plutôt d'un magazine littéraire hebdomadaire dont nous serions institués lecteurs. Composé d'une dizaine de séquences, régulières ou pas, permettant d'approcher le livre et l'auteur sous plusieurs angles : des entretiens en tête-à-tête sur le plateau de Poivre d'Arvor avec des auteurs venus présenter leur livre, mais aussi avec d'autres auteurs ou critiques venus donner leurs coups de cœur, des reportages montrant les écrivains chez eux ou sur les sites qui les inspirent, des documents ou images d'archives ressuscitant les grands classiques au gré des anniversaires et des rééditions... Les différentes séquences de l'émission, qui constituent proprement les rubriques du magazine sont introduites par la répétition du même procédé utilisé pour le générique: une page est tournée portant l'intitulé de la rubrique à suivre (Exploration, Ex-térieur livre, Ex-pliquez moi...).

La diversité des rubriques ne saurait cependant dissimuler l'omniprésence de PPDA ; elle la rend au contraire d'autant plus sensible : «Présent à tout bout de champ, aussi bien plongé dans les archives que transplanté aux antipodes, la gravité de chacun de ses tête-à-tête n'a d'égale que la multiplicité de ses goûts ${ }^{34}$. "

Le voici dans les appartements de Benazir Bhutto, chef d'État pakistanais, pour un entretien exclusif à propos de ses mémoires à peine parus... On se croirait au journal de 20 heures $^{35}$.

De retour sur le plateau, le voici encore qui présente ses invités :

PPDA - C. Juliet, nous étions quelques-uns à le connaître à travers son Journal chez Hachette ; et il vient de publier enfin L'Année de l'éveil.... ${ }^{36}$

PPDA - Et maintenant, un éditeur que j'ai adoré (i.e. Guy Schoeller), à travers deux livres de lui, édités par lui, que j'ai emmenés avec moi pendant les vacances de Noël : Maupassant, que j'ai lu et relu' ${ }^{37}$.

Le voici maintenant qui introduit à la séquence "Ex-ploration »: consacrée à des écrivains disparus et faite d'images d'archives dont il assurera qui plus est le commentaire.

PPDA - Joseph Kessel, on est obligé de l'aimer. Moi, je sais que quand j'avais quinze ans, je lui ai écrit un petit mot et il m'a répondu une lettre formidable que j'ai toujours gardée par-devers moi, et depuis vingt-cinq ans, je ne cesse de penser à lui ${ }^{38}$.

PPDA - Antonin Artaud, un homme au destin assez exceptionnel, que je vais vous raconter ${ }^{39}$.

PPDA - Et maintenant, Alexandra David-Neel, avec une interview réalisée cinq semaines avant sa mort ; c'était il y a exactement vingt ans puisqu'elle est décédée en 1969. C'est elle qui en 1926 avait fait ce formidable voyage à Lhassa, le Voyage d'une parisienne à Lhassa ; j'ai passé mon dimanche en sa compagnie ${ }^{40}$.

Le voici enfin qui nous livre ses coups de cœur: «ces nouvelles que j'ai lues tout au long du week end dernier ».

PPDA - J'en ai profité pour relire, pour lire d'ailleurs, car je dois vous dire que je n'avais pas lu, je n'avais pas lu Salman Rushdie. Les Enfants de minuit. Je vous recommande infiniment Les Enfants de minuit.

PPDA - Je vais vous recommander d'acheter Les Femmes et la Révolution, parce que c'est un texte de Michelet que je ne connaissais pas.

71 Si la forme d'Ex-libris est celle d'un magazine littéraire, c'est bien d'un véritable livre qu'il s'agit, dont les chapitres sont composés des différentes rubriques de l'émission. Un livre en plusieurs volumes, qui ne sont autres que les émissions successives et dont le 
personnage principal est un lecteur. Après Bartleby l'écrivain, voici « PPDA le lecteur " dans des aventures toujours renouvelées qui sont autant d'histoires vécues, de fragments autobiographiques. Les livres dont il est question, aussi nombreux et divers soient-ils, PPDA les a lus ; mieux, il les a emmenés avec lui, il était avec eux ce dimanche encore. Sa lecture des œuvres n'est pas ici simplement présupposée, elle est constamment exhibée et rapportée au moment de son effectuation. "J'ai passé mon dimanche en sa compagnie.» Tout se passe alors comme si c'était cette expérience personnelle qui donnait également leur prix aux livres présentés ; car PPDA les a tous lus et donc aimés. Au point qu'il nous recommande tel ouvrage précisément parce qu'il ne le connaissait pas: il vient de le lire et c'est une raison suffisante pour le recommander! De ce point de vue, l'intitulé de l'émission se justifie pleinement: les livres présentés semblant tout droit tirés de la bibliothèque de PPDA.

Le livre fictionnel Ex-libris, ce journal intime d'un lecteur considérable, se lit comme un véritable livre d'aventures. A mi-chemin entre «Connaissances du monde... des lettres" et "l'être le plus extraordinaire que j'aie jamais rencontré", il nous fait partager les pérégrinations de "PPDA le lecteur" aux quatre coins du monde, et ses rencontres avec des personnages hors du commun. La présentation d'Ex-libris par la speakerine de service est d'ailleurs sans équivoques: "Mélanger les genres pour multiplier les paysages et donner toute sa dimension à l'aventure littéraire, c'est la spécialité de Patrick Poivre d'Arvor qui laissera ce soir s'exprimer grands écrivains, astrophysiciens, poètes, grands reporters et baroudeurs. " Et maintenant, en route pour l'aventure ${ }^{41}$.

PPDA - Bonsoir ! Les droits de l'homme en question dans cette émission qui va nous emporter un peu partout dans le monde : en Israël avec l'écrivain A. Oz qui était Prix Fémina étranger cette année ; en Roumanie avec le caricaturiste M. Stanescu ; dans le monde entier avec A. de Marenches qui fut, vous le savez, le patron des services secrets pendant plus de dix ans; et tout de suite, en Argentine avec $\mathrm{H}$. Bianciotti ${ }^{42}$.

PPDA - Bonsoir! Nous avons décidé ce soir de consacrer cette émission à l'aventure, l'aventure sous toutes ses formes. Alors d'abord, des aventuriers puis des aventuriers du livre dans la seconde partie de l'émission. Les aventuriers : le moins qu'on puisse dire, c'est qu'ils ont réalisé des performances assez extraordinaires. Alors, vous entendrez F. Varigas, qui lui, revient du Grand Nord, T. Monod, qui lui, revient du Sahara, J. Pasteur qui revient de l'Amazonie péruvienne, puis R. Bricka, qui lui, a traversé l'Atlantique à pied ou presque ! Et puis, il y aura un hommage à la pionnière, Alexandra David-Neel, qui est, vous le savez, la première européenne a avoir pénétré à Lhassa au Tibet en 1926. Et puis, il est d'autres formes d'aventures ! L'aventure du livre, avec Guy Schoeller qui est un des hommes qui a inventé le livre de poche, celui qui a inventé la collection «Bouquins» chez Robert Laffont. également $\mathrm{H}$. Polies, dont la bibliothèque est exceptionnelle et le parcours aussi exceptionnel.

Avec un premier invité, tout à fait exceptionnel. Rassurez-vous, vous êtes toujours dans une émission littéraire! [R. Bricka, homme-orchestre de profession, nous gratifie d'un remarquable numéro de saltimbanque avant de venir répondre aux questions de PPDA sur son livre ${ }^{43}$.

PPDA - Bonsoir! Dans la vie de certains êtres, il y a parfois des déclics, et la vie ordinaire devient extraordinaire. C'est ce qui est arrivé à tous mes invités de ce soir. Paul-Emile Victor a eu envie un jour de tailler la route : il est parti, vous le savez, vers les pôles et puis, de l'infiniment froid il est passé à l'infiniment chaud ; il vit aujourd'hui à Bora Bora. C'est là que nous l'avons rencontré pour le faire parler de la bibliothèque de ses rêves. Bernard Clavel lui aussi, on peut dire qu'il a pris ses jambes à son cou puisqu'il vit aujourd'hui en Irlande et il vient d'achever le dernier 
tome de sa saga sur le Canada. Et puis, S. Hawking! Alors S. Hawking, c'est un personnage exceptionnel. Nombreux sont ceux qui disent de lui que c'est déjà le nouvel Einstein. Il a quarante-huit ans, il vit déjà depuis une vingtaine d'années sur une petite chaise roulante; il ne peut pas s'exprimer et nous l'avons rencontré à l'université de Cambridge.

Et puis Nicolas Hulot (i.e. présentateur vedette d'un magazine de l'aventure sur TF1), lui aussi a eu une destinée exceptionnelle. On peut peut-être dire que tout a basculé un jour de décembre 1973 parce que votre frère est mort dans des conditions qui vous ont marqué à tout jamais et c'est peut-être de ce jour-là qu'est née votre révolte qui vous a emmené aux quatre coins du monde ${ }^{44}$.

73 Avec Ex-libris, la vieille opposition du livre et de la télévision se résout dans la simultanéité de l'audience et de la lecture; non pas, chose inconcevable, cette lecture exécutée sur le plateau et dont nous serions les spectateurs passifs, mais une lecture opérée par le téléspectateur lui-même. Or, la condition de possibilité d'un tel tour de force tient dans le type de définition de la pratique lectorale ici mis en œuvre: lire, c'est s'évader, partir à la rencontre d'auteurs qui sont eux-mêmes hommes d'aventures. Détachée de la temporalité de la réception, la lecture se réalise ici dans l'immédiateté de la rencontre, dans l'instantanéité du dépaysement. Le temps de la lecture est aboli : c'est ce qui permet à PPDA de tout lire le week end (alors qu'il est pris le reste de la semaine par la préparation de «son journal»); c'est ce qui permet encore la transformation des téléspectateurs que nous sommes, en lecteurs.

Au terme de l'émission, en partie consacrée à C. Juliet, PPDA présente dans le cadre de la rubrique "Ex-tras et ordinaires ", le résultat d'une enquête exclusive CSA-Télérama-Exlibris, réalisée du 2 au 6 février auprès d'un panel représentatif de trois cents lecteurs de nouveautés du livre.

PPDA - L'heure des coups de cœur maintenant, à commencer par vos coups de cœur ; c'est-à-dire les coups de cœur des trois cents personnes interrogées très régulièrement par notre magazine, Télérama et CSA, qui nous permettent tous les mois de voir ce que vous avez aimé ou pas. Cela n'est pas forcément la liste des bestsellers ; c'est plutôt une appréciation de la qualité... [Antoine Blondin obtient 17/20, H. Bianciotti 15,5/20]. On peut évoquer au passage C. Juliet, que les lecteurs ont lu avant les téléspectateurs [sic !], puisque vous êtes en train de le découvrir dans cette émission : $14 / 20^{45}$.

\section{Apostrophes : le livre à grand spectacle}

Tout a été dit, ou presque, sur Apostrophes, l'émission de Bernard Pivot créée en janvier 1975 et devenue proprement synonyme d'«émission littéraire ». On s'est d'abord unanimement félicité d'une audience sans précédent pour ce genre d'émission, répercutée de façon spectaculaire sur les ventes, à la grande satisfaction du monde de l'édition. La littérature disait-on "sort enfin de son ghetto ». Certains ont pu voir les raisons d'un tel succès dans la personne même de Bernard Pivot: nombre d'articles soulignent tour à tour, sa bonhomie, son espièglerie ou son intégrité, un ouvrage lui est consacré $^{46}$, une bande dessinée le prend pour héros ${ }^{47}$... Au point que Pivot lui-même s'en inquiète: "On s'intéresse de plus en plus à Pivot et de moins en moins à Apostrophes ${ }^{48}$." «Le vendredi, avait dit François Truffaut, je regarde Apostrophes, le samedi et le dimanche, ce sont les jours où je vois ma famille, et le reste du temps je travaille » et Les Cahiers du cinéma d'interroger tout naturellement le dispositif scénique de ce qu'on a coutume d'appeler « la dramatique du vendredi soir ${ }^{49}$ ». 
Beaucoup, intellectuels pour la plupart, ont au contraire manifesté leur défiance face à un succès qui ne pouvait que se payer d'une banalisation culturelle. D'autres encore et non des moindres, tel Régis Debray, alors conseiller à l'Elysée, ont stigmatisé « un monopole à la fois du choix des titres et du choix des auteurs, accordé finalement à l'arbitraire d'un seul homme qui exerce une véritable dictature sur le marché du livre ». Plus circonspects, H. Hamon et P. Rotman considèrent pour leur part qu'Apostrophes constitue le strict reflet des transformations internes au petit monde de l'intellocratie ${ }^{50}$. A quoi bon, objectera-t-on, poser ici les termes d'une controverse dans laquelle nous avons déjà manifesté la volonté de ne pas nous engager? Simplement pour établir qu'il est un présupposé commun aux différents points de vue, impliqué par l'objet même de la controverse, à savoir 1'«effet-Apostrophes». Se féliciter qu'Apostrophes fasse vendre des livres, regretter au contraire que les livres présentés relèvent d'un arbitraire ou encore que leur contenu soit à peine effleuré, voilà qui présuppose en effet que l'émission, comme émission "littéraire » est au service du livre. Tandis que du propre aveu de Bernard Pivot : «Ce n'est pas la télévision qui est au service de la littérature ; c'est la littérature qui est au service de la télévision ${ }^{51}$.»

Reconnaissons-le d'emblée, parce qu'il ne s'agit pas là d'un effet de l'émission mais de sa prémisse, il est indiscutable qu'Apostrophes fait lire... Bernard Pivot. L'éditeur Alain Mo-reau le dit tout net: «Si les autres émissions littéraires sont mauvaises c'est que leurs animateurs ne lisent pas. Quand on écoute Bernard Pivot, on a la conviction qu'il ne s'est pas contenté de parcourir le livre, de réciter le prière d'insérer ou de répéter ce que lui a expliqué une attachée de presse. On ne surprend pas Bernard Pivot à sortir des bourdes ou des généralités bavardes ${ }^{52}$. » Bernard Pivot le confesse volontiers: " La meilleure manière de faire mon métier, c'est d'être chez moi, lire les livres et ne pas fréquenter les écrivains. ${ }^{53}$ " « Mes choix ne sont fonction que de l'intérêt littéraire que je trouve aux livres. Je ne pense absolument pas aux problèmes de télégénie des écrivains. Il m'est arrivé récemment de réunir cinq auteurs que je n'avais jamais vus. Seul m'a guidé l'intérêt de leurs livres ${ }^{54}$."

Mais ne nous y trompons pas, le métier de Bernard Pivot n'est pas celui du critique littéraire. C'est d'abord un homme de télévision dont l'objectif clairement conçu et ouvertement revendiqué est d'assurer un spectacle: "On me reproche de faire du spectacle, mais la télévision est spectacle. On peut me reprocher de faire du bon ou du mauvais spectacle, mais pas du spectacle. Comment faire à la télévision quelque chose qui ne soit pas du spectacle puisqu'il est déjà dans l'œil du téléspectateur? La télévision a inventé son langage. Il est réducteur et stimulant ; il a des défauts et des qualités. Les défauts: on n'explique pas en profondeur un livre comme un critique littéraire peut le faire sur quatre colonnes. Il ne s'agit pas de transcrire la critique littéraire de la presse écrite à la télévision. Les qualités : l'auteur se défend lui-même en présentant son livre ${ }^{55}$.»

79 Autrement dit, la formule originale d'Apostrophes - consacrer chaque émission à un ensemble de livres qui traitent plus ou moins d'un même thème et dont les auteurs s'entretiennent mutuellement - ne résulte d'aucune invention. Elle est tout simplement une formule contrainte, imposée au genre de l'émission littéraire par le «langage » propre de la télévision, la solution incontournable au dilemme du producteur d'émissions littéraires : faire du spectacle avec des choses inertes! «Un livre ça ne bouge pas. Alors, j'ai choisi de passer par les auteurs pour donner cette dimension spectaculaire ${ }^{56}$.» Dans cette perspective, c'est au réalisateur de l'émission qu'il 
appartient, « en s'interdisant de passer autre chose que des plans significatifs [sic!], de transformer une conversation, qui peut être la plus intelligente du monde mais qui est une conversation, en spectacle ${ }^{57}$ ».

Tel est le paradoxe d'Apostrophe: si c'est bien le livre que Bernard Pivot invite sur le plateau... c'est l'auteur qui y fait le spectacle. Certes, la présence physique du livre sur le plateau joue un rôle essentiel comme administration de la preuve de la légitimité de la présence de l'auteur sur le plateau; encore cette fonction légitimante ne requiertelle de l'objet livre que son format parallélépipédique et une couverture. Le livre alors exhibé est littéralement un livre sans contenu, qui pourrait dans ces conditions tout autant être fictif, comme le fut d'ailleurs bel et bien cet ouvrage présenté un $1^{\text {er }}$ avril par Bernard Pivot, intitulé Le Vrai dans le faux et le faux dans le vrai, attribué à Raymond Devos, et que nombre de téléspectateurs de l'émission devaient s'étonner, les jours suivants, de ne pas trouver en librairie. Les formes concrètes de l'exhibition nécessaire du livre sont au demeurant des plus restreintes.

81 Sur le plateau tout d'abord, l'objet-livre est l'affaire exclusive de Bernard Pivot. Pendant toute la durée de l'émission, il a un livre (bien) en main, marqué de signets qui attestent de sa propre lecture et sont autant d'amorces possibles pour sa conversation avec l'auteur. Le dialogue se poursuit aussi longtemps que le livre en question n'est pas rangé et remplacé par un autre, Bernard Pivot changeant alors d'interlocuteur. D'autres livres sont en piles à ses côtés; en fin d'émission, il les prendra tour à tour pour en dire quelques mots tout en présentant leur couverture à la caméra. Les auteurs invités, pour leur part, n'ont pas de livre! Mais quel usage Bernard Pivot fait-il du livre qu'il garde avec une telle constance par-devers lui ? Essentiellement celui-ci : pendant que l'auteur à l'écran répond à sa précédente question, il consulte les notes portées au crayon au dos de la couverture de manière à préparer la suivante qu'il lui adressera ensuite en le regardant, comme si elle lui était venue spontanément, dans le cours de la conversation. L'alternance systématique à l'écran, du champ (sur Pivot questionnant)contre champ (sur l'auteur répondant) entretenant cette fiction conversationnelle. Aussi lorsque Bernard Pivot ouvre le livre, c'est le plus fréquemment sur une page blanche; il s'en sert alors comme d'un carnet de notes, d'un aide-mémoire. Plus rarement, il peut en extraire une citation, toujours brève, servant à présenter le portrait condensé d'un personnage ou une formulation concise de la thèse soutenue par l'auteur, en prémisse au questionnement.

Cependant, comme le fait justement remarquer P. Lejeune: "Tout effet d'écriture dépassant la dimension de la phrase a tendance à disparaître, ou se trouve noyé dans la vague notion de "style". A Apostrophes, l'allusion au "style" de l'écrivain sert souvent de clausule à son tour de piste. Quand Pivot lui coupe la parole, ouvre impatiemment le livre qu'il tenait à la main et déclare : "Et maintenant, votre style", on sent que c'est fini. Après le fond, la forme... Ici, le style est, bien sûr, admiré, ou au pire cité sans commentaires. On a parfois l'impression que Pivot a choisi son échantillon un peu au hasard, il le lit cursivement et platement, et il lui arrive d'enchaîner la dernière phrase de la citation (il ferme le livre, en prend un autre et se tourne dans une autre direction) avec la première phrase qu'il adresse à l'auteur suivant ${ }^{58}$. »

En d'autres termes, quand survient la lecture du texte, pour thématiser cette question du style, supposée extra-conversationnelle, elle constitue une manière de « rituel de fermeture " dans l'échange avec l'auteur. On ne saurait manifester plus clairement l'altérité radicale du régime de la lecture au régime souverain de la conversation 
auquel peut en revanche se soumettre la brève citation. La référence littérale au texte doit, en effet, satisfaire aux fins pratiques de la conversation. En ce sens, le livre aux mains de Bernard Pivot, devient un ressort dramaturgique mobilisable à tout moment dans sa stratégie globale d'animation du plateau.

A propos du grand marché européen de 1993 :

Bernard Pivot - Vous annoncez à la France le maximum de difficultés.

A. Minc - Pas de difficultés...

B. P. - Comment?

A. M. - Mais d'adaptations, oui un petit peu.

B. P. - Ah, vous annoncez le maximum de difficultés! Alors là... Vous voulez que je vous lise là (sûr de lui).

A. M. - Faites, faites.

B. P. - Non, non, parce que vous annoncez clairement les choses là ; j'ai été surpris.

A. M. - Non, non.

B. P. - Attendez, attendez... Ah, page 162 (lit en levant l'index) : «La France aura plus de difficultés que les autres $»^{59}$ !

Par ailleurs, pendant que l'auteur est interrogé par Bernard Pivot, le livre de référence est à plusieurs reprises, à l'écran cette fois, présenté en plan fixe, le téléspectateur n'en voyant que la couverture. Néanmoins, suivant une technique de montage originale (également adoptée aujourd'hui par Ex-libris), l'auteur en train de parler sur le plateau fait son apparition par une fenêtre ménagée au milieu de la couverture, au-dessous de son nom et du titre du livre. Comme le note encore P. Lejeune: "Cette astuce de montage a pour fonction de relier le plan fixe à l'image du débat (on se sert aussi parfois de la surimpression) : mais elle a en même temps une valeur allégorique. » Tout se passe alors en effet comme si le livre n'avait d'autre contenu que l'auteur en train de parler sur le plateau d'Apostrophes; comme s'il ne se réalisait qu'en cette occasion; comme si la chose inerte qu'il était s'animait enfin. Le contenu réel du livre, quant à lui, est escamoté par cet effet d'instantané ; tout au plus, s'il y a lieu, une main invisible tournant les pages nous montrera-t-elle des illustrations, jamais du texte. Ainsi, quand c'est l'existence proprement textuelle de son livre qui justifiait la présence de l'auteur sur le plateau, c'est la prestation en direct de l'auteur qui donne finalement au livre son contenu ! Le dispositif de l'émission qui résultait jusque-là d'un impératif pragmatique (assurer le spectacle en dépit de l'inertie du livre), opère symboliquement une transformation substantielle du livre. Le texte en tant que tel n'a plus d'importance; il ne consiste plus que dans les idées exprimées par son auteur. Comme si tous les livres étaient des livres d'idées, les auteurs simplement des personnes ayant des idées sur un certain sujet, et la littérature elle-même un débat d'idées. Émission "littéraire ", Apostrophes ne l'est qu'à ces conditions; son principe d'ordre thématique ayant précisément pour fonction d'instaurer un débat autour d'idées censées exister à l'intérieur des livres que leurs auteurs présentent mais qui existent aussi, bien au-delà et plus largement, dans la société. Telle est du moins la conviction à l'origine du projet de l'émission, de l'aveu de Bernard Pivot: "J'étais attiré par la réalisation d'une émission thématique, qui regrouperait des écrivains sur un même sujet. Déjà, avec Ouvrez les guillemets, je me rendais compte de toutes ces lignes de force de l'édition qui faisaient, qu'à un certain moment, des gens traitaient le même thème. Ce n'est pas une volonté des éditeurs, là aussi vous avez des pulsions souterraines chez les intellectuels ; ça vient de l'université, ça vient de l'évolution de la société et, à un moment, ça émerge sous forme de livre. Rappelez-vous, en septembre 1977, sept livres sur la mort. Une émission centrée autour d'un thème me permettait d'organiser un débat ${ }^{60}$. Ainsi 
conçu, le livre est un révélateur dont la valeur est d'abord une valeur sociale qui excède la singularité propre du livre et se tient comme telle, au contraire, dans une communauté thématique dont il n'est qu'une composante. Davantage qu'une émission littéraire, Apostrophes apparaît alors comme un véritable documentaire sur l'actualité intellectuelle, voire, plus encore, comme une scène privilégiée de sa constitution comme telle par l'intermédiaire d'une mise en débat d'un genre particulier. En effet, le prétendu débat instauré sur le plateau d'Apostrophes ne saurait porter sur le contenu des œuvres ni consister dans la confrontation des thèses et points de vue respectifs des différents auteurs. D'ailleurs, ces derniers ne l'ignorent pas, comme en témoigne cet échange entre deux universitaires invités chacun pour un livre sur la question de 1 '« Étranger ».

Après que $T$. Todorov, en réponse aux questions de Bernard Pivot, a présenté son ouvrage, Julia Kristeva fait part de son désaccord concernant la lecture par Todorov des textes de Diderot et Montaigne consacrés à 1'« Etranger ».

B. P. - Alors, Todorov? [i.e. que répondez-vous à ça ?].

T. Todorov - Je pense que nous ne trancherons pas ici.

J. Kristeva - Il faudrait un colloque du CNRS pour le livre de Todorov.

T. T. - Ce sont des écrivains qui ont des facettes multiples; il faut discuter avec les

textes en main ; on ne va pas le faire maintenant ${ }^{61}$ !

87 En fait de débat, dans le cadre thématique propre à chaque émission, il s'agit plutôt d'établir un système de renvois entre les différents ouvrages présentés le plus souvent hétérogènes, de manière à légitimer leur présence respective et commune sur le plateau. A cet effet, chacun a reçu pour consigne de lire avant l'émission le livre des autres auteurs invités; en sorte que les livres d'autrui valent comme autant de ressources conversationnelles. C'est ce que montrent ces fragments d'une émission réunissant Jean Dutourd de l'Académie française, dont le dernier ouvrage stigmatise la propension journalistique au cliché, François Weyergans, romancier, G. Miller, psychanalyste qui dans son livre analyse les lapsus des hommes politiques, L. Fournier, auteur d'un pamphlet sur la vie sexuelle des hommes politiques et enfin Pétillon, dessinateur pour le dernier volume des aventures de Jack Palmer, détective incompétent ${ }^{62}$.

A propos du livre de François Weyergans:

B. P. (à G. Miller) - Est-ce que les écrivains, finalement, n'écrivent pas pour échapper à la psychanalyse ?

G. Miller (à F. W.) - C'est vrai que c'est un livre qui est très traversé par la psychanalyse.

F. Weyergans - C'est ça oui, traversé.

B. P. (à G. M.) - Non, mais ce que je veux dire, c'est que l'écrivain, au lieu de se confier au psychanalyste sur son divan, il se confie à la page blanche.

F. W. - C'est pas du tout la même chose.

B. P. - Parce que vous avez fait des psychanalyses, vous?

F. W. - J'ai fait les deux, alors je peux parler : ça n'a rien à voir...

A propos du livre de Jean Dutourd :

G. Miller - Est ce qu'il y a des clichés de Jean Dutourd lui-même ; parce qu'on ne les relève pas dans votre livre, que j'ai lu attentivement.

J. Dutourd - Parce que je n'en emploie pas.

B. P. - Ah si ! Il y a un adjectif dont vous raffolez...

J. D. - Oui, c'est « épatant».

B. P. - Voilà !

J. D. - Et vous savez de qui je le tiens? 
B. P. - Non.

J. D. - De Jean Paulhan... il mettait même le superlatif : c'est très épatant!

A propos du héros de la bande dessinée de Pétillon, détective incompétent et gaffeur :

B. P. (à G. Miller) - C'est quand même un superbe personnage.

G. Miller - C'est un superbe personnage... Il y a un côté «névrose obsessionnelle » chez Jack Palmer.

L. Fournier interrompt G. Miller qui vient d'interpréter quelques lapsus commis par des hommes politiques, pour lui signaler un lapsus qu'il a lui-même commis dans son livre ; il en propose une interprétation hâtive saluée par des bravos ironiques et par cette exclamation : mais, c'est le Jack Palmer de la psychanalyse !

91 La lecture n'est pas ici opérateur de classement et de préférences; elle est un impératif pragmatique requis aux fins pratiques de la conversation. Elle est, comme telle, régie au contraire par un principe d'indifférenciation et de neutralité. S'il est vrai, comme l'avance P. Bourdieu, « qu'il est probable qu'on lit quand on a un marché sur lequel on peut placer des discours sur la lecture ${ }^{63} "$, alors le plateau d'Apostrophes est bien le théâtre d'un rituel de socialisation du livre.

\section{Le livre sans la lecture}

Emblématique chacune d'un type de solution pratique apporté aux problèmes de la contradiction entre lecture et télévision, les émissions sur les livres considérées cidessus peuvent, par là-même, faire l'objet d'une lecture diachronique qui les inscrive chacune dans un cadre de problématicité spécifique (i.e. les représentations successives du média impliquant à chaque fois un rapport particulier à la culture).

De ce point de vue, Lecture pour tous, tout comme Lire c'est vivre apparaissent comme des émissions proprement littéraires, à vocation culturelle, caractéristiques de cette mission de service public qui fut celle des pionniers de la télévision. Dans un cas comme dans l'autre, il s'agit bien en effet de faire accéder les téléspectateurs au monde de la culture, en les familiarisant dans un cas, avec ces « vaches sacrées » (pour reprendre le titre d'une émission de Françoise Giroud s'inscrivant également dans le même cadre) que sont les écrivains, en leur présentant dans l'autre, des lecteurs exemplaires auxquels s'identifier.

Avec Libre et change, succédant à Droit de réponse en perpétuant le même esprit contestataire qui, au lendemain de Mai 1968, animait déjà Post-scriptum (la toute première émission de critique littéraire de Michel Polac, interrompue en mai 1971 après une émission « à scandale » sur l'inceste), la télévision n'est plus cet opérateur de révélation, d'initiation. Elle est au contraire devenue un instrument de domination et d'imposition culturelles, d'autant plus efficace qu'il engage à la consommation passive. Pour autant que sa supposée transparence le cède à l'obstacle, la télévision demeure ce média par l'intermédiaire duquel il s'agit encore de faire accéder le plus grand nombre à une culture qui existe déjà, dans toute son intégrité indépendamment du média. Simplement, la perspective critique transforme en relation dialectique la relation unilinéaire qu'établissait le modèle de la (télé) diffusion culturelle (la télévision au « service public » du livre). C'est à partir d'une critique de la télévision ordinaire que la culture peut être convoquée à la télévision sans y perdre sa substance. Inversement, c'est à partir d'une critique de la culture convoquée par la télévision ordinaire que la télévision peut échapper à sa propre insignifiance. 

l'image des chaînes, l'idée est désormais inscrite, en termes de "mieux-disant culturel » dans le cahier des charges des différentes chaînes. On a vu le profit qu'Antenne 2 retire d'Apostrophes, on a vu aussi ce que la création d'Ex-libris sur TF1 doit à de telles considérations. Cependant, ainsi instrumentée, la culture ne désigne plus une réalité substantielle extra-médiatique; elle est un label attribué à certaines émissions : ce n'est plus la télévision qui est au service du livre mais, comme on l'a dit, le livre qui est au service de la télévision.

Ces reformulations du rapport problématique qu'entretiennent culture et télévision sont autant de cadres de pertinence successifs, constituant chacun un type spécifique de présentation du livre en solution pratique au problème générique des émissions sur les livres. Dans ces conditions et en dépit de leur nombre restreint, les émissions considérées nous permettent de saisir les transformations implicitement à l'œuvre d'un type d'émission à un autre et qui sont indissociablement celles de la représentation du livre et de la signification du fait lectoral.

97 A Lectures pour tous, comme à Lire c'est vivre, le livre est un donné dont l'évidence est celle de la chose écrite et dont la réalité est garantie par ce monde de la culture (dite précisément livresque) auquel il est supposé se rattacher. Objet culturel précédant la lecture, le livre doit être examiné dans sa textualité. La lecture, pour sa part, est dans ces conditions figurée comme une expérience singulière de confrontation intime à la textualité des œuvres.

Inversement, à Libre et change, l'existence même du livre est thématisée comme un enjeu fondamental, emblématique qu'elle est d'une démocratie réputée être la condition nécessaire de la culture. Aussi le livre y est-il d'abord célébré, ici encore antérieurement à toute lecture, du strict point de vue de sa factualité. La lecture, pour sa part, est alors figurée comme une mise à l'épreuve collective, par la communauté intellectuelle, de la textualité des œuvres devenue chose dé-battable, conformément à l'idéal démocratique.

Enfin, à Apostrophes, avec le recadrage dans le livre de la performance de l'auteur sur le plateau, tout comme à Ex-libris, avec la constitution de l'émission en méta-livre, le livre ne se réalise plus que dans son accomplissement télévisé; tandis que la lecture se confond désormais avec la réception, collective, de l'émission.

$\mathrm{Au}$ terme de cette brève lecture diachronique des formes typiques de la présentation du livre (dans les émissions ayant vocation à présenter le livre), et sans préjuger des dispositifs formels à venir, il convient d'accorder une attention toute particulière à l'efficacité, unanimement reconnue, de la formule d'Apostrophes, longtemps tenue, et aujourd'hui encore ${ }^{64}$ pour indépassable, et ce faisant, de reconsidérer la question de l'audience.

101 Rappelons au préalable que les téléspectateurs les plus assidus se trouvent parmi les détenteurs de niveaux faibles d'instruction, majoritaires chez les dits "faibles lecteurs ${ }^{65}$. C'est dans ce cadre qu'il faut peut-être apprécier le remarquable succès d'audience d'une émission comme Apostrophes (pour autant que son public dépasse justement le cercle des lecteurs confirmés et $a$ fortiori celui des acheteurs). On peut faire l'hypothèse que c'est pour avoir démarqué l'émission littéraire tant du débat intellectuel (cf. Libre et change), que de la relation d'une expérience intime (cf. Lire c'est vivre), qu'Apostrophes peut concerner bien au-delà du public captif (intellectuels et gens 
de culture d'un côté, lecteurs de l'autre) attaché à ces deux formes canoniques de l'émission littéraire. En fait de démocratisation du rapport à la lecture, il s'agit là de la manifestation la plus spectaculaire d'un processus conjoint de collectivisation du rapport au livre et de détachement par rapport à la lecture. Pour ceux-là qui ont en commun d'avoir suivi à la télévision la même présentation d'un livre par son auteur, la possibilité de tenir des discours concernant les lectures (c'est-à-dire, si l'on suit P. Bourdieu, de socialiser le rapport à la lecture) n'est plus fonction de la lecture effective : on ne discute plus du livre qu'on a lu mais de l'émission qu'on a vue.

En réalisant une socialisation du 1ivre qui fait l'économie de la lecture, Apostrophes résout par conséquent cette hétérogénéité que nous avions posée d'entrée comme principielle, des cadres temporels respectifs de la lecture et de la télévision (durée/ immédiateté). Celle-là même que les dispositifs des émissions précédentes n'avaient eu de cesse de neutraliser, en figurant la lecture, tout d'abord, dans la conscience de l'écrivain (Lectures pour tous), puis dans le livre (Lire c'est vivre), ou encore en prenant la lisibilité même comme thème (Libre et change). Et voici que la lecture finit par s'abolir dans le présent de la réception télévisée.

Ce que révèle en somme l'approche en diachronie des formes successivement instaurées aux fins pratiques des émissions dites «littéraires", c'est une double tendance, de détachement du livre par rapport à la lecture, et de dissociation entre réception et lecture. Toute paradoxale qu'elle puisse paraître, cette tendance se confirme en synchronie avec l'extension de la présence du livre à d'autres scènes que celle de l'émission littéraire.

\section{Le livre à tout bout de champ}

La coïncidence est remarquable: le livre devient objet télévisuel omniprésent au moment où Apostrophes, émission qui n'a plus de littéraire que le nom, règle enfin la double contradiction inhérente au principe même de l'émission littéraire. Soit la contradiction entre les cadres temporels respectifs de la lecture et de la télévision, et la contradiction entre le projet littéraire, impliquant d'accéder à la textualité et la normativité télévisuelle interdisant la lecture. Comme on l'a vu, la condition d'une telle résolution tient dans l'abandon du projet de figuration de la lecture, jusque-là tenu pour consubstantiel au projet même de l'émission littéraire, en vertu d'une identité implicite entre lecture et culture. On peut dès lors avancer l'hypothèse suivante: pouvant par principe ménager en leur sein une place au livre, parce qu'à l'abri de telles contradictions, les émissions autres que littéraires s'y engagent effectivement à la condition que le livre cesse d'être défini, en sa totalité, comme objet culturel par ces instances de définition attitrées que sont les émissions littéraires. Alors, et alors seulement, le livre à la télévision peut être soumis au régime commun de la référence, événementielle ou biographique, et satisfaire ainsi aux exigences de ces deux genres télévisuels par excellence que sont les émissions d' «actualité » et les émissions de "variétés " $^{66}$. Et si le livre est encore convoqué à des fins de légitimation culturelle, celle-ci est désormais assurée par une référence explicite à telle émission littéraire. 


\section{Le livre aux Jeux du stade : le sport cultivé 67}

105 C'est une idée bien établie, le sport et la culture ne font pas bon ménage et on imagine mal un sportif lisant... autre chose que L'Équipe. Dans ces conditions, la présence du livre dans un magazine sportif comme Les Jeux du stade a de quoi surprendre. C'est pourtant une présence bien établie en période de fêtes de fin d'année, comme le rappelle le présentateur D. Casais : "Vous le savez, c'est un peu une tradition aux Jeux du stade de vous présenter des livres pour Noël. Quel plus beau cadeau que le livre ! Et des livres de sport, il y en a beaucoup et certains sont très beaux. » En d'autres termes, si les sportifs ne lisent pas, du moins pourront-ils offrir ! Ainsi est-ce par une procédure de désacralisation que le livre, devenu objet marchand, fait son entrée sur le plateau des sports. Quitte ensuite à retrouver son prestige : «La semaine dernière, je vous ai présenté des livres-cadeaux, cette semaine c'est davantage des livres à lire, des livres à se procurer pour les garder. » Finalement, le livre sportif serait donc un livre comme les autres, également digne d'être lu ; un objet culturel à part entière. Si le livre pénètre comme objet marchand sur le plateau, comme pour tromper la défiance d'un public supposé faible lecteur, c'est pour y (re)devenir de plus belle opérateur de légitimation culturelle. « Et maintenant, le quart d'heure culturel du service des sports ! J'ai l'insigne honneur d'accueillir sur ce plateau le libraire du service des sports d'Antenne 2, que dis-je le pivot... Bernard Pivot, bien sûr. » La référence à l'émission littéraire n'est pas ici simple boutade: dans la bouche du journaliste ainsi commis au rôle de Pivot d'occasion, les livres sportifs seront présentés à la manière d'ouvrages de littérature ; L'Année du cyclisme de Pierre Chagny, par exemple : "C'est un classique, qui se répète un peu tous les ans, mais c'est un très bon auteur; un très bon livre tout à fait indispensable dans une bibliothèque sportive... il est vrai que le cyclisme, c'est un petit peu un roman de cape et d'épée... "

\section{Le livre au journal télévisé : en prise directe avec l'actualité}

Sur la scène du journal télévisé, le livre ne saurait être que livre d'actualité et ce, de bien des manières. Première manière : le livre par lui-même peut faire l'actualité. Ce fut le cas très récemment avec les Versets sataniques, dont la publication est à l'origine de la dite "affaire Rushdie », véritable "chronique d'une mort annoncée », qui devait faire longtemps la une des "actualités", alors même que le livre et l'auteur nous demeuraient étrangers. Question de vie ou de mort encore que celle suscitée par la publication, voici quelques années, de Suicide, mode d'emploi. Sous le titre "Suicide en vente libre", un magazine d'actualité avait consacré un reportage à ce qui était présenté comme une véritable question de société : "Faut-il laisser en librairie les recettes de la mort ${ }^{68}$ ? On pouvait voir en particulier le mari d'une victime lire, le livre à la main, certains passages annotés par sa femme.

Le déclic qui lui a fait acheter ce livre, je ne pourrais jamais le connaître; par contre, il est bien certain que la lecture d'un tel ouvrage par quelqu'un de faible, ça entraîne à faire ce qu'elle a fait. Elle souligne encore [gros plan sur la phrase soulignée qu'il s'apprête à lire], page 260 , je vais terminer là-dessus : «Et si le suicide était une solution... ». Pendant quatre mois, on s'est acharné à lui redonner le goût à la vie, on avait presque réussi; elle a eu le livre en main, elle s'est suicidée! 

intéresse l'émission de variétés. Tandis que l'un relativise l'intérêt du livre en le rapportant à l'actualité immédiate, l'autre remet en cause la fonction-auteur traditionnelle. Que se passe-t-il en effet lorsque des ouvrages sont présentés dans le cadre d'émissions de variétés (Champs-Élysées, Sacrée soirée...) ? Davantage qu'un auteur, c'est la vedette, l'homme de spectacle bien connu par ailleurs des téléspectateurs qui vient faire la présentation de son livre, dont il n'est qui plus est, le plus souvent, que le signataire. Dans la plupart des cas, le bref entretien que lui consent l'animateur de l'émission est centré sur la surprise de retrouver une Linda de Souza en auteur; à moins que, plus rarement (Signoret, Bohringer...) on n'insiste sur le fait qu'il s'agit d'un «vrai » livre. Le paradoxe alors n'en est que plus grand. Dans l'exemple qui suit, l'animateur de l'émission (A la folie, pas du tout) n'est autre que Patrick Poivre d'Arvor, 
actuel présentateur d'Ex-libris; preuve que la frontière entre les genres n'est plus ce qu'elle était !

P.P.D.A. - Vous allez voir que nous avons des invités de marque, avec tout d'abord H. Schumacher, le gardien de but de l'équipe d'Allemagne. [Des images nous le montrent en pleine action... Suit une liste de personnalités du monde du spectacle au terme de laquelle H. Schumacher, premier invité de l'émission, en costume de ville, descend sous les applaudissements du public, un escalier d'honneur pour venir s'asseoir aux côtés de PPDA qui peut alors entamer l'interview.] M. Meyer, qui est le directeur de l'information à Radio France, est venu avec vous, il vous a aidé à accoucher d'un livre qui vient de sortir à l'instant et qui s'appelle Coup de sifflet.

De ce livre, qui révèle l'existence de pratiques de dopage dans le milieu du football professionnel, il ne sera plus question, sinon pour dire que Schumacher s'est montré « assez courageux en l'écrivant ${ }^{71} »($ sic).

L'incongruité du rôle endossé sur cette scène est telle que le dit «écrivain " peut tout autant réussir son examen de passage médiatique en recourant à cette forme particulière de " distance au rôle » qu'est l'autodérision. Exemplaire à cet égard est la prestation de Daniel Prévost, (acteur comique bien connu des téléspectateurs, puisqu'il fut l'animateur d'une émission de divertissement) sur le plateau de Sacrée soirée, émission de variétés championne toutes catégories du taux d'audience ${ }^{72}$.

Jean-Pierre Foucault (présentateur vedette de l'émission) - Vous savez que bien souvent dans cette émission, nous nous connaissons, ce qui était le cas de Michel (i.e. Michel Sardou, chanteur vedette, qui vient d'interpréter son dernier succès); ce qui va être le cas de la personne que nous allons accueillir. Ça fait longtemps que nous ne nous étions pas vus. On avait fait les fous pendant une émission qui s'appelait L'Académie des neuf; depuis on ne s'était plus revus... Voici mon ami Daniel Prévost. Daniel Prévost!

[D. P. apparait sur le plateau et salue le public, sous ses applaudissements.]

D. P. - Messieurs-dames, je vous demanderais de reprendre vos applaudissements.

[Seconde salve d'applaudissements entrecoupée de rires.]

J.-P F. - Daniel, je suis très content de vous recevoir parce qu'on vous connaît bien mal.

D. P. - Moi aussi.

J.-P F. - Et de vous, nous allons parler dans quelques instants.

D. P. - Oui, je préfère qu'on parle de moi quand je suis là [rires].

J.P. F. - Il vaut mieux, généralement dans ce métier, il vaut mieux... Je vais vous demander cher ami de vous livrer à un exercice indispensable au bon déroulement de l'émission, c'est-à-dire nous raconter un événement important de votre vie.

D. P. - Écoutez, j'ai eu beaucoup d'événements dans ma vie, comme tout le monde, mais l'événement le plus important, celui qui a compté le plus dans ma vie, il est clair que ce fut incontestablement le jour de ma naissance [rires]...

J.-P. F. - Très bien; et puis vous avez su rester vous-même, à travers quelques mots simples et choisis.

D. P. - C'est toujours très simple ce que je dis.

J.-P. F. - J'ai remarqué... Je vous ai suivi longtemps faire mon métier, c'est-à-dire animer un jeu télévisé qui s'appelait Anagrammes, qui a eu un succès considérable...

D. P. - Considérable!

J.P. F. - ... sauf pour les dirigeants de la télévision.

D. P - Oui, mais ça, vous savez: ils sont passés, je reste [rires mêlés d'applaudissements]. Je vous demanderais Mesdames, Messieurs, d'observer une minute de silence pour eux [rires].

J.-P. F. - Je ne dirai rien car les miens sont encore là.

D. P. - Peut-être pas pour longtemps non plus [rires].

J.-P. F. - S'il vous plaît, on ne s'égare pas Daniel. Le théâtre, puisqu'il y a trois 
semaines j'ai reçu Balutin qui est votre compère dans Drôle de couple. On ne va pas dire ce qu'a dit Balutin.

D. P. - Il l'a peut-être mal dit [rires]. C'est une comédie extrêmement amusante que nous jouons depuis plus d'un an au théâtre Saint-Georges... Parlons de tout ce que vous voulez en dehors de... De tout ce que vous voulez.

J.-P. F. - Parlons littérature (un livre apparaît aussitôt à l'écran en surimpression d'un gros plan sur D. P.).

D. P. - Alors là, c'est autre chose ! [en regardant la caméra l'index levé].

J.-P. F. - Parlons du Prévost qu'on connaît un peu moins [il présente l'ouvrage à la caméra; sur la couverture, il y a une photo couleur de D. P. avec le même sourire sarcastique qui est le sien sur le plateau] avec ce Journal intime et inutile d'une vie banale (il se tourne vers D. P. tout en continuant de présenter le livre à la caméra).

D. P. - Oui, c'est exact. Alors écoutez, je sais parfaitement que vous avez... que vous n'avez pas beaucoup de temps à vous, que vous ne l'avez pas lu. Alors, je l'ai lu pour vous [hors champ, rire de J.-P. F qu'on voit ensuite en plan cut baisser le livre] et j'ai fait une fiche de lecture.

J.-P. F. - Merci beaucoup.

D. P. - Non non. Mais il n'y a rien de désagréable, je sais parfaitement que vous êtes très occupé. D'abord, vous apprenez à lire et c'est très long [rires dans le public]. Alors voilà, j'ai fait une fiche de lecture pour vous parler de mon livre [il manque d'éclater de rire]. Tout d'abord, je dois dire incontestablement que le titre m'a beaucoup amusé : Journal intime et inutile d'une vie banale, c'est très amusant ; et il est de moi bien entendu [plan cut sur J.-P. F. qui présente le livre]. Ensuite, je peux vous dire autre chose, c'est que j'ai lu mon livre d'une traite et c'est irrésistible : c'est plein d'humour, c'est plein de tendresse [il en lit un passage]... C'est le livre d'un humoriste, vous voyez, qui traite un petit peu de tous les sujets sérieux avec évidemment une réflexion un petit peu tendre, un petit peu profonde sur tous les sujets de ce monde [il recommence la lecture]... Voilà, je parle également dans ce livre de...

J.-P. F. - Pourrait-on arriver à la conclusion?

D. P. - Y'a pas! Il n'y a pas de conclusion. Non, ce que je veux dire c'est que c'est un livre qui apporte du bonheur, parce qu'on a besoin de bonheur dans cette crise, dans ce monde de crise où les valeurs spirituelles sont niées, où les intégristes catholiques vont par exemple faire des choses violentes contre le film de Scorcese.

J.-P. F. - Non, je...

D. P. - Si ! Comment non? Vous n'habitez pas ce pays ou quoi [rires]. Alors, tout ça...

J.-P. F. - Je voudrais simplement dire que...

D. P. - Oui, dites-le !

J.-P. F. - A travers la dérision qu'on peut entendre dans vos propos, Daniel Prévost, moi je vous conseille vivement la lecture ; même si vous dites que je ne l'ai pas lu et c'est pas vrai, la lecture de votre livre.

D. P. - Je plaisantais.. Vous avez lu le titre et c'est déjà très important [rires].

J.-P. F. - Laissez-moi parler, maintenant.

D. P. - Allez-y, allez-y.

J.-P. F. - Laissez-moi parler de vous. Moi, j'avais lu votre premier livre qui s'appelait Coco, belles nattes. C'est vrai, j'avais lu votre premier livre [plan cut sur D. P., yeux fermés, humble, comme pris sur le fait] et là, j'avais découvert un Prévost qui est parfaitement à l'opposé de l'image qu'il veut donner de lui. J'ai lu cette... en deux mots, cette amitié profonde qui liait deux gamins. Vous pouvez me rappeler, mais franchement, en deux mots.

D. P. - Oui, oui, d'accord, bon, c'était deux petits garçons dont l'un est juif et l'autre pas, qui se sont trouvés dans les années d'après-guerre dans une période troublée. C'est vrai, j'ai écrit ça, oui, bien sûr.

J.-P. F. - C'est un bouquin autobiographique, c'est-à-dire que le juif est quelqu'un qu'on ne connaît pas et l'autre c'est Daniel Prévost, on est bien d'accord. 
D. P. - [fermant les yeux] C'est un petit peu moi.

J.-P. F. - Laissez-moi parler parce que je sais que vous n'aimez pas qu'on parle de vous, en tout cas dans ces termes. Le petit juif, il a grandi, je l'ai fait venir ce soir pour vous parce qu'on a plein de choses à se dire. Voici Yankl!

[Y. apparaît sur le plateau, à la grande surprise de D. P. ; ils s'embrassent et répondant à la sollicitation de J.-P. F. ; Y. raconte l'histoire émouvante de ces deux enfants qui se défendaient mutuellement. D'abord très ému, D. P. se met à grimacer ostensiblement au fur et à mesure du récit, jusqu'à interrompre Y. en riant.]

D. P. - Mais attends, ils vont pas acheter le bouquin ! [il rit].

J.-P. F. - En tout cas, merci Yankl d'être venu nous faire découvrir un Prévost qu'on ne connaît pas.

D. P. - C'est extra! En tout cas... Franchement nous sommes tous très touchés, la France entière est très touchée. Maintenant, je voudrais vous faire une surprise.

J.-P.F. - Qu'est-ce que c'est?

D. P. - Moi de mon côté, j'ai préparé pour vous spécialement un poème [il sort de sa poche un papier qu'il lit] : à Jean-Pierre Foucault :

«Quand le mercredi soir devant ma grosse télé affalé dans le fauteuil je te vois arriver aux lèvres un grand sourire de gorille joyeux dans ton joli costume qui en contiendrait deux comme moi, je me dis quelle surprise encore Jean-Pierre a préparée?

Est-ce un reste de porc qu'il offrira ce soir à cette belle actrice au visage de madone et aux, excusez-moi c'est écrit en petit, aux énormes cuisses

A-t-il appris par cœur une page de mon Journal intime et inutile d'une vie banale

M'aura-t-il retrouvé un vieil arrière, arrière, arrière-grand-père descendant de Robespierre ou Marat

Allez mon cher Jean-Pierre, ça ira, ça ira.

Je vous laisse à présent, j'économise ma gorge car le public m'attend au théâtre Saint-Georges. » [tonnerre d'applaudissements]

J.-P. F. - Merci Daniel, merci.

114 Ce n'est pas en premier lieu l'écrivain qui est accueilli sur le plateau, mais par ordre d'entrée en scène : l'ami, le collègue animateur de jeux télévisés, l'homme de théâtre, et enfin seulement l'écrivain. De même, ce n'est pas lui non plus qui quitte le plateau, mais l'acteur attendu par le public au théâtre Saint-Georges. Tout se passe dans ces conditions comme si le rôle d'écrivain ne pouvait être assumé sur cette scène qu'encadré par des rituels d'ouverture et de fermeture d'entretien, rituels de reconnaissance, confirmant pour leur part l'identité de la vedette. Car c'est précisément l'incongruité même d'un tel rôle pour la vedette (et la mise en scène de cette incongruité) qui permet de ménager une place au livre dans la dramaturgie d'une émission dont le principe est justement de révéler la face cachée des vedettes. Pas question ici de présenter un livre ; il suffit de présenter un « écrivain », et dévoiler ainsi la véritable personnalité supposée cachée derrière la façade publique de la vedette. Inversement, pour la vedette invitée, la promotion de son livre nécessite, de la même façon, qu'il reste fidèle à son image de marque.

En conséquence, c'est une véritable parodie d'émission littéraire qui s'engage par un très emphatique autant qu'inattendu: "parlons littérature ", auquel répond le refus d'occuper la place de l'écrivain manifesté sur le mode de l'autodérision, par un invité qui prend celle du présentateur en se faisant lecteur de son propre livre. «Parler littérature ", c'est alors ni plus ni moins que parvenir à poser, contre toute attente, les présupposés des émissions littéraires : "C'est vrai que j'ai lu votre livre ", "C'est vrai que j'ai écrit ça ». Cela suffit, puisque aussi bien, il ne s'agit pas ensuite d'interroger l'auteur sur son livre. La vérité du livre n'est pas dans l'auteur, c'est la vérité de l'auteur qui est dans le livre. Plus exactement, le livre en tant qu'il a pu être écrit par la vedette 
témoigne simplement d'une sensibilité jusqu'ici méconnue, qu'il va s'agir de restituer sur le plateau, par exemple en ménageant à la vedette invitée des retrouvailles avec un ami d'enfance, banalisant ainsi ce contenu documentaire du livre qui était précisément supposé faire toute sa valeur.

Comme s'il devait y avoir une différence de nature entre les émissions littéraires et les autres, on a coutume de déplorer que le livre vienne se compromettre sur d'autres scènes télévisuelles que celle-là qui lui est assignée. Nous avons vu au contraire qu'il ne s'agit là que de la diffusion massive, dans l'espace audiovisuel, d'une orientation déjà nettement marquée dans l'évolution des émissions littéraires elles-mêmes. Selon le même présupposé implicite, on reconnaît généralement le bénéfice exclusif d'un tel processus aux émissions autres que littéraires (comme une manière de légitimation culturelle). Ceci tout en regrettant que les émissions littéraires, de plus en plus, soient asservies à l'actualité éditoriale, et cèdent à la vedettarisation des auteurs. C'est oublier que pour être «culturelles ", elles n'en sont pas moins « émissions » comme les autres et qu'à ce titre, c'est leur légitimité proprement «télévisuelle » qu'elles assurent en empruntant, pour leur part, aux genres canoniques de la télévision que sont les actualités télévisées et les émissions de variétés.

Que les livres présentés dans les différentes émissions, littéraires ou non, s'inscrivent effectivement dans une "actualité du livre » (comme il y a une actualité sportive que peuvent tout autant suivre les spectateurs pratiquants ou non), c'est ce que confirme une étude comparative portant sur l'intervalle existant entre la date de publication des ouvrages et leur date de passage à l'antenne dans les différents genres d'émission ${ }^{73}$.

Chez Pivot, au moins, pourra-t-on objecter, on est sûr de ne trouver que de véritables auteurs, des écrivains (peu de chances d'y voir Sulitzer). Ici, nulle ambiguïté ne doit planer sur le bien-fondé de l'appartenance de tel invité au cercle des écrivains et si besoin, la rapide lecture d'un morceau choisi suffira à lui donner droit de cité (Signoret, Mitterrand...). Pour autant, c'est précisément sur cette scène qu'est à l'œuvre la remise en cause la plus profonde de la fonction-auteur traditionnelle. Les écrivains sont réunis pour présenter leur livre en parlant. Ils n'ont pas par exemple la possibilité d'en lire un extrait, ils doivent parler et même, si le plateau est bien constitué, converser. Leur livre est là, chose inerte, et il tient à la performance de l'auteur devenu porte-parole, de lui donner vie.

\section{NOTES}

1. Éliseo Véron, «Le séjour et ses doubles ; architectures du petit écran », Temps libre, 1984, $\mathrm{n}^{\circ} 11$ : "Les médias ne sont pas des dispositifs de reproduction d'un réel qu'ils copient plus ou moins exactement mais bel et bien des dispositifs de production de sens.» L. Quéré, Des miroirs équivoques, Aubier, 1982. A propos de l'information télévisée: le savoir produit est en même temps « reflet et substitut du référent, il semble reproduire le réel alors qu'il l'institue de part en part » [...]. «Le spectateur contemple le sujet de l'énonciation, il juge non pas ce qu'il dit et fait, 
mais sa manière d'énoncer, son talent, son authenticité, sa personnalité, ses sentiments. » (p. 158).

2. Sur le processus historique de «désocialisation » de l'acte de lecture, de recouvrement d'une lecture à voix haute par une lecture silencieuse, pour soi-même, on pourra se reporter aux travaux de R. Chartier.

Aujourd'hui, comme le souligne justement P. Bourdieu, c'est a posteriori que se socialise la pratique lectorale, dans un marché «sur lequel on peut placer des discours, concernant les lectures ». « Comprendre les pratiques culturelles », Pratiques de la lecture, op. cit.

3. P. Arnaud «Littérature : le spectacle impossible », 25 images/seconde - rencontres télévision Valence, catalogue 1989 (cité dans Les Dossiers de l'audiovisuel, $\mathrm{n}^{\circ}$ spécial «Littérature et télévision ", janvier-février 1990).

4. Bernard Pivot ne croit pas si bien dire : «Tout compte fait, une émission comme Apostrophes, si elle désacralise les hommes et les femmes qui y paraissent, aux yeux du public elle les sacre écrivains. » Entretien avec Bernard Pivot, propos recueillis par S. Daney et S. Toubiana, Cahiers du cinéma, $\mathrm{n}^{\circ}$ spécial « Télévision », automne 1981.

5. Roland Barthes, « Réponses », Tel quel n 49, 1971.

6. Libération, 27 septembre 1985.

7. Océaniques, F. Poirier, P.-A. Boutang.

8. « Portrait-souvenir : François Mauriac », ORTF, 1962.

9. Annie Ernaux, «L'écrivain en terrain miné », Le Monde, 23 mars 1985.

10. Apostrophes, émission spéciale consacrée à Vladimir Nabokov, 30 mai 1975.

11. Océaniques, « 52 minutes dans la langue », L. Trovato, J.-P. Thibaudet.

12. Entretien avec Bernard Pivot, Cahiers du cinéma, op. cit.

13. Si l'on excepte Apostrophes: plus de six cent soixante émissions (à ce jour) sur la même période de 15 ans, de 1975 à 1990.

14. Pierre Dumayet, «L'interview télévisuelle », Communications, 1966.

15. Lectures pour tous, François Mauriac, ORTF, 1959.

16. Entretien avec Pierre Dumayet, Cahiers du cinéma, op. cit.

17. Ibid.

18. Lecture pour tous, Louis-Ferdinand Céline, ORTF, 1957.

19. «Je ne sais pas si l'interviewé a le sentiment de s'adresser à un public. Nous savons bien que celui-ci existe, mais on ne s'adresse pas à lui directement. J'ai plutôt l'impression que le public est témoin s'il le souhaite. » Pierre Dumayet. «L'interview télévisuelle », op. cit.

20. Lire c'est vivre, Germinal, Antenne 2, 18 septembre 1984.

21. Libre et change, 14 juin 1989.

22. Libre et change, 17 mai 1989.

23. Libre et change, 14 juin 89.

24. Télérama, 19 octobre 1988.

25. Ibid.

26. Libre et change, 24 mai 1989.

27. Libre et change, 14 juin 1989.

28. Libre et change, 5 avril 1989.

29. Cf. M. Agulhon. Le Cercle dans la France bourgeoise (1810-1848) : étude d'une mutation de sociabilité, Armand Colin, 1977. Sur le rôle de la lecture dans la constitution de l'espace public, $c f . J$. Habermas, L'Espace public, Payot, 1978.

30. Libre et change, 17 avril 1989.

31. Le Figaro, 26 octobre 1988.

32. Télé 7 jours, 9 octobre 1988.

33. Télérama, 19 octobre 1988.

34. J. Prieur, « Littérature et télévision », Dossiers de l'audiovisuel, n²9, 1990, p. 13. 
35. Ex-libris, 12 avril 1989.

36. Ex-libris, 15 février 1989.

37. Ex-libris, 18 janvier 1989.

38. Ex-libris, 8 février 1989.

39. Ex-libris, 15 février 1989.

40. Ex-libris, 18 janvier 1989.

41. Cette expression, on peut aussi bien l'entendre en son sens propre grâce au concours Ex-librisTF1-Le Point : chaque semaine, deux questions sont posées aux téléspectateurs de l'émission. Les vainqueurs, tirés au sort, gagnent un voyage pour deux personnes, pour partir par exemple «sur les traces de Victor Hugo à Guernesey et à Jersey »!

42. Ex-libris, $1^{\text {er }}$ février 1989.

43. Ex-libris, 18 janvier 1989.

44. Ex-libris, 22 février 1989.

45. Ex-libris, 15 février 1989.

46. E. Brasey, L'Effet Pivot, op. cit.

47. Pétillon, Les Disparus d'Apostrophes, Éd. Dargaud.

48. Le Matin, 23 septembre 1985.

49. Les Cahiers du cinéma, $n^{\circ}$ 50, Télévision, 1981.

50. P. Hamon, H. Rotman, Les Intellocrates, Complexe, 1985.

51. Très précisément, ce propos extrait d'une interview donnée au Figaro du $1^{\mathrm{er}}$ avril 1974 concerne la précédente émission de Bernard Pivot: Ouvrez les guillemets. Cette formule convient néammoins tout aussi bien à Apostrophes.

52. Propos rapportés dans Antennes, décembre 1980.

53. Le Matin, 23 septembre 1985.

54. Télé 7 jours, 26 mai 1981.

55. Les Nouvelles littéraires, 26 juillet 1979.

56. Le Monde, 6 février 1977.

57. Propos de J. Cazenave, réalisateur d'Apostrophes recueillis par Libération du 27 septembre 1985.

58. P. Lejeune : « L'image de l'auteur dans les médias », Pratiques, $n^{\circ} 27,1980$.

59. Apostrophes, 13 janvier 1989.

60. Les Nouvelles littéraires, 26 juillet 1979.

61. Apostrophes, 24 février 1989.

62. Apostrophes, 9 juin 1989.

63. P. Bourdieu, R. Charrier, « Comprendre les pratiques culturelles », op. cit., p. 224.

64. Il n'est que de voir le désarroi suscité par l'annonce de la disparition prochaine de l'émission. Cf. « Que faire après Apostrophes », Livres-Hebdo n 9, 1990.

65. Les Pratiques culturelles des Français, 1982, op. cit. p. 55.

66. D'autant que, comme le signalait déjà une enquête du Figaro du 13 mai 1975, les maisons d'édition apprécient tout particulièrement « l'émission qui parle de livres sans être une émission proprement littéraire », touchant ainsi, comme par surprise, un autre auditoire que le public captif des émissions littéraires.

67. Les Jeux du stade, 21 décembre 1985, 22 décembre 1986.

68. Infovision, 20 septembre 1984.

69. Soir 3, 24 novembre 1980.

70. A2-13 heures, 22 juin 1987.

71. A la folie, pas du tout, 14 juin 1987.

72. Sacrée soirée, 19 octobre 1988.

73. On trouvera en annexes (I) divers tableaux de résultats en pourcentage. Pour le premier semestre 1989 : les données concernant les livres passés : à Apostrophes (tab. 1), à Ex-libris (tab. 2), à Libre et change (tab. 3) ; les données concernant les livres passés dans ces trois émissions 
littéraires (tab. 4). Pour le premier semestre 1980 : les données concernant les livres passés : à Apostrophes (tab. 5), les livres passés dans les émissions littéraires (tab. 6) ; l'ensemble des livres passés à la télévision (tab. 7) ; les livres passés au journal télévisé (tab. 8) ; les livres passés plus d'une fois à la télévision (tab. 9). Pour 1989, nous avons systématiquement recherché dans LivresHebdo la date de publication de tous les livres passés dans l'une des trois émissions littéraires. Pour 1980, nous avons de surcroît pu consulter une liste établie par l'INA, seule manière pour nous de comptabiliser les passages des livres hors émission littéraire (d'autant que ce type de passage n'est pas annoncé dans les magazines de programmes ni même dans les périodiques spécialisés du type Livres-Hebdo). Hélas ce type de recensement exhaustif n'est pas encore réalisé par l'INA pour les années récentes. 


\section{Chapitre 3. L'auteur en scène : de l'écrivain à l'« homme de parole ${ }^{1}$ "}

Aux dernières maisons de la rue - aujourd'hui il était arrivé jusque-là - la défaite de l'écrivain se trouva scellée: d'abord quelqu'un derrière lui l'appela et, se retournant involontairement, il se vit photographié ; puis un homme habillé de noir

lui barra le chemin, leva l'index et proclama : «Votre littérature, je la suis pas à pas ! »; enfin il

y en eut un qui, sans le regarder, exigea : «Un autographe pour mon enfant! » Pendant qu'il s'exécutait (souhaitant, il est vrai, avoir pour cela

un troisième bras mécanique) il lui sembla ne plus être du tout un écrivain, contrairement à ce qu'il avait éprouvé pendant l'heure qui avait suivi

le travail, mais d'en jouer seulement le rôle, ridicule et contraint ; avoir eu besoin de réfléchir avant de signer pour que son nom lui revienne, n'en était-ce pas la marque ? Par ailleurs, se dit-il, c'était bien fait pour lui, c'est lui qui avait permis qu'on connût son visage. Mais s'il avait la possibilité de recommencer son métier, il ne devait plus y avoir un seul portrait de lui. » (Peter Handke, L'Après-midi d'un écrivain, Gallimard, 1988)

\section{Quand l'auteur paraît}

\section{Y a-t-il un écrivain sur le plateau?}

1 Professeurs, journalistes, autobiographes de tous ordres, mais aussi romanciers à vocation tardive: les auteurs de circonstance ont investi massivement la scène 
littéraire... au détriment, peut-on penser, des écrivains dits classiques (véritables ?)². Voilà qui semble porter à son comble la fameuse désacralisation de l'écrivain qui résulterait, aux dires mêmes de Bernard Pivot, de sa médiatisation. Prudence cependant en matière d'imputation causale : autrefois déjà, Cervantès n'était-il pas soldat, Claudel ambassadeur ou Céline médecin ? Est-ce à dire, dans ces conditions, que la nouveauté maintes fois signalée du phénomène tiendrait simplement à son ampleur, ou encore au type d'activité exercé, aujourd'hui, par les prétendants au titre d'écrivain?

2 Reconsidérons plutôt la réponse apportée par le même Bernard Pivot à l'argument de la désacralisation : " Tout compte fait, une émission comme Apostrophes, si elle désacralise les hommes et les femmes qui y paraissent, aux yeux du public, elle les sacre écrivains. » L'émission littéraire apparaît alors comme une instance de désignation, d'étiquetage... d'autant plus efficace et requise que les hommes et les femmes en question sont des auteurs de circonstance. Elle constitue en effet, comme telle, un lieu privilégié de résolution symbolique d'une crise profonde de la condition d'écrivain, dont elle contribue à manifester les termes contradictoires que sont d'une part une absence de statut de l'écrivain, d'autre part un « devenir-écrivain " généralisé ; et ce en faisant de leur confrontation même un principe dramaturgique ${ }^{3}$. Même s'il n'est pas dans notre propos de développer ici ce point, il convient néanmoins de préciser plus avant ces deux dimensions contradictoires de la crise en question.

3 D'une part, du point de vue juridique, l'écrivain n'existe pas. Certes, la loi du 11 mars 1957 modifiée par la loi du 3 juillet 1985 (loi Lang) protège les œuvres littéraires, mais au même titre ni plus ni moins que les œuvres musicales, théâtrales ou cinématographiques, toutes également qualifiées d'« œuvres de l'esprit ». Par ailleurs, depuis la loi du 31 décembre 1975, complétée en 1977, les auteurs disposent d'une Sécurité sociale propre, gérée par l'Association pour la gestion de la sécurité sociale des auteurs (Agessa). Cependant, il faut disposer d'un minimum de droits d'auteur (fixé à 1200 fois le Smic comme plancher horaire) pour bénéficier de cette prestation sociale. En sorte que l'Agessa ne comptabilise pas plus de mille deux cent trente écrivains, définis comme tels par la Sécurité sociale, dont seulement trois cent quarante-six peuvent vivre de leurs revenus (9 400 francs bruts, par mois, de droits d'auteur)... alors même que la Société des gens de lettres (SGL) regroupe près de onze mille membres. Enfin, depuis la réforme de 1974, les droits d'auteur sont assimilés à des salaires. Cependant si l'imposition peut être étalée sur trois ou cinq ans, si l'auteur bénéficie depuis la loi de finances de 1984 d'une déduction forfaitaire de $25 \%$ pour frais professionnels dont le montant est plafonné à 50000 francs, l'écrivain n'a toujours pas de retraite complémentaire. Pour toutes ces raisons, un véritable statut de l'écrivain est au centre des revendications rassemblées par la SGL dans un « livre blanc $»^{4}$.

4 Or, ce vide statutaire constitue d'autre part l'arrière-fond d'une véritable « invasion des graphomanes ", pour reprendre le titre d'un article d'Alain Finkielkraut ${ }^{5}$, dont de larges extraits suffiront à donner la mesure de cette seconde dimension de la crise actuelle de la condition d'écrivain.

C'est le chaos dans la république des belles-lettres, naguère si policée. Quelques rares élus déployaient les fastes de leur style devant un public connaisseur, admiratif et muet; mais voici maintenant qu'un vouloir-écrire généralisé, épidémique, s'empare des populations.

Dans notre société de consommation, qu'on dit volontiers gouvernée par les deux seuls principes de l'argent et du plaisir, hommes et femmes de (presque) toute 
condition noircissent fiévreusement des pages, consacrant ainsi une part appréciable de leur temps libre à un travail désagréable, harassant, angoissé, et dont, pour la plupart d'entre eux, ils ne retireront aucun profit matériel. Tournant le dos aux séductions de l'hédonisme, la multitude des graphomanes a choisi, dans le silence et la solitude, de se torturer pour rien...

C'est moins sans doute le désordre que la banalité qui pousse à écrire : mis en mots, le quotidien devient intéressant, le trivial apparaît unique, et la vie journalière échappe à sa propre insignifiance. Une platitude écrite n'est plus une platitude, et la prouesse verbale communique un peu de son état à l'existence, qu'elle est censée seulement dépeindre.

«La forme coûte cher », dit le poète. C'est indéniable, mais elle rapporte gros. L'immortalité ? Oh, non! Notre époque n'est pas si ambitieuse. L'éternité est une valeur en baisse et puis il faut parer au plus pressé : on s'efforcerait peut-être de prendre une assurance contre la mort si on avait l'assurance préalable d'avoir vraiment vécu. Le néant des temps morts est bien plus effrayant que la mort future, et c'est cette anxiété que beaucoup d'entre nous conjurent en écrivant...

Et ceux-là mêmes que rebutent les complaisances de l'écrivain intime ne deviennent jamais graphomanes que pour se dédoubler : docteurs Jekill et misters Hyde de nos sociétés quotidiennes, ils rachètent leur moi de tous les jours par une subjectivité ténébreuse et légendaire : celle de l'écrivain...

Il faut donc beaucoup d'hypocrisie pour reprocher aux médias (c'est-à-dire, en fait, à la télévision) d'avoir perverti la littérature en y introduisant le vedettariat. Qu'est-ce que la gloire moderne et versatile d'une émission comme Apostrophes auprès du prestige insigne attaché depuis deux siècles à la fonction (on n'ose pas dire au métier) d'écrivain?

Ce mana, cette puissance symbolique et sacrée, il fallait autrefois la partager avec d'autres professionnels du livre. Désormais, l'écrivain est seul : le charisme des professeurs, pour citer un exemple, n'a pas survécu à la démocratisation et à la crise de l'école. D'ailleurs, on ne dit plus professeur, mais enseignant : cette cruauté lexicale traduit, mieux que tout discours, la banalisation de la parole pédagogique. une fonction sacrée est devenue, en quelques années, intégralement profane. Cette baisse brutale de statut ne met pas fin à toutes les joies du métier (d'autres plaisirs peuvent naître d'une complicité plus grande avec les élèves), mais quoi d'étrange, cependant, à ce qu'il y ait tant de graphomanes parmi les enseignants? Ceux-ci veulent, par l'écriture, prendre leur revanche sur une carrière sans surprise, ponctuée par les visites inopinées de l'inspecteur et bercée par les rêves, sans cesse déçus, de mutation.

5 Dans les termes d'une telle analyse, on pourra en revanche reprocher sans la moindre hypocrisie aux médias (c'est-à-dire à la télévision - c'est-à-dire à Apostrophes -) de sacrer écrivain, puisque aussi bien telle est la fonction que le "grand prêtre » Pivot reconnaît à Apostrophes; c'est-à-dire de sacrer effectivement «écrivains", et à tour de bras encore, les dits « graphomanes» qui ne demandent pas mieux. Or, c'est précisément cette prétendue efficacité télévisuelle - d'autant moins discutée ici qu'elle permet de confondre à bon compte deux phénomènes hétérogènes, la prolifération de l'écriture comme expérience intime, l'invasion de la scène littéraire par des hommes de lettres d'un nouveau genre -que nous entendons mettre en cause.

6 Considérant cette même question de l'ouverture de la "république des belles lettres ", mais pour notre part, du strict point de vue de son accomplissement sur la scène publique, nous ignorerons la catégorie du " graphomane » qui renvoie à d'improbables coulisses, pour nous tenir à celle d'«auteur» telle qu'elle est, au contraire, littéralement constituée sur la scène médiatique : l'auteur n'est autre que le répondant de son livre. C'est-à-dire, comme on le verra, tour à tour et tout à la fois : celui qui porte la responsabilité du livre, qui en répond, et celui qui s'exprime en lieu et place du livre, 
qui doit avoir du répondant. De même, n'aurons-nous pas à postuler quelque définition positive de l'écrivain: cela reviendrait à poser l'écrivain, avec une idolâtrie de graphomane, en figure référentielle incontestable, en label invariant dont les modalités d'acquisition seraient simplement renouvelées ${ }^{6}$, mais à dégager la définition qu'en donne implicitement le dispositif télévisuel, en creux de sa définition en positif de l'auteur.

\section{Le livre en quête d'auteur}

Certes la crise actuelle de la condition d'écrivain est avant tout une crise statutaire dont les déterminants sont d'ordre extramédiatiques. Soit, d'un point de vue interne, une indétermination dans la définition juridique du statut de l'écrivain, et d'un point de vue externe, une recomposition du champ intellectuel et plus largement, le devenir hégémonique d'un modèle expressif de l'accomplissement individuel. Il nous reste néanmoins à en spécifier les effets proprement télévisuels.

8 Tout d'abord, la télévision prend une part active dans l'élargissement du cercle des hommes de lettres, en inventant et promouvant un genre inédit d'auteur: le présentateur de télévision lui-même. Ainsi, la confusion des rôles est-elle à son comble lorsque PPDA, par ailleurs présentateur vedette du journal télévisé reçoit à Ex-libris son habituel collaborateur $\mathrm{du}$ « 20 heures» en la personne de Michel Cardoze, " présentateur-météo » bien connu pour arborer une fleur chaque jour différente à sa boutonnière et agrémenter ses prévisions d'une citation poétique. L'actualité du livre est alors télévisuelle de part en part: hors contexte, le présentateur du journal de 20 heures reçoit monsieur Météo. Tandis que c'est le romancier méconnu du grand public qui est attendu sur le plateau, c'est bien monsieur Météo que l'on retrouve, avec sa fleur à la boutonnière, et qui termine la présentation de son livre par une petite phrase d'Edmond Jabès. Tandis que c'est le romancier qu'avait accueilli PPDA, c'est à monsieur Météo qu'il donne l'au revoir : «C'est une jolie fin, on a l'impression d'être à $20 \mathrm{~h} 40$. Merci beaucoup Michel Cardoze et à demain soir ${ }^{7} . »$

Et surtout, comme nous l'avons précédemment avancé, l'émission sur les livres est le lieu par excellence de l'objectivation des problèmes de statut, puisqu'elle présuppose une dissociation de principe entre l'auteur de circonstance et son livre (le livre étant un attribut paradoxal pour un auteur qui se définit par ailleurs). Il est dans ces conditions pour le moins excessif, même si conforme au sens commun, de prétendre qu'une émission comme Apostrophes puisse sacrer quiconque écrivain (définition ontologique, renvoyant à un sujet unitaire); tout au plus désigne-t-elle publiquement des auteurs (définition fonctionnelle, renvoyant à un sujet fragmentaire). Le plus souvent, en effet, ce n'est pas un écrivain qui est invité sur le plateau mais un livre, objet inerte devant être accompagné d'un tuteur (dont la présence sur le plateau est donc une implication fonctionnelle de la présence première du livre) qui soit son porte-parole. En d'autres termes, l'invitation du livre sur le plateau ne requiert pas l'existence préalable d'un écrivain ; elle institue, au contraire, un auteur implicitement conçu comme la personne qui assume la responsabilité du livre sur la scène publique. Or, cette position de l'auteur ne peut paradoxalement être occupée que par une personne dont l'identité sociale singulière aura été préalablement confirmée (accusant ainsi, dans le cas d'un auteur de circonstance, la question de la factualité de son livre). Présenter le livre, c'est alors le rapporter à cette identité sociale de l'auteur et à la compétence qui peut lui être 
attachée, ou encore à sa biographie; le rapporter en bref à ce principe de vérité référentielle que constitue un auteur pareillement institué. Exemplaire est ici la présentation, sur le plateau d'Apostrophes, d'un certain Mirko Grmek, auteur d'une Histoire du sida.

Bernard Pivot (présentant ses différents invités) - Mirko

Grmek, vous êtes un savant !

[...]

B. P.-Mirko Grmek, venons-en à votre livre. C'est une histoire remarquable. Moi, j'ai appris évidemment beaucoup de choses que j'ignorais totalement. [...] Moi, ce qui m'a impressionné, dans votre livre, c'est, je dirais, le côté policier de l'affaire ; c'està-dire comment les médecins ont peu à peu appris à savoir comment ça se transmettait... [...]

M. G. - Ce qui m'a paru particulièrement important, c'était d'écrire avec le plus de simplicité mais sans concessions aucune. Vraiment, tout ce que j'ai écrit correspond à l'état actuel de la recherche.

B. P. - Je l'ai compris de A à Z votre livre; donc là je suis un bon cobaye ${ }^{8}$

On le voit, la présentation concertée du livre fait littéralement émerger au rang de critère d'appréciation déterminant la question de la vérité référentielle qui, si elle s'énonce ici, s'agissant d'un "savant», en termes de rigueur scientifique, est plus largement affaire de sincérité; et dont la «façade » personnelle de l'auteur invité, devenue surface expressive transparente, est le miroir. C'est en fonction de ce critère qu'un « auteur » est institué, tel qu'il puisse assumer la responsabilité du livre sur la scène publique. Ici c'est parce que l'invité est confirmé en sa qualité de savant qu'il peut ensuite se révéler auteur d'un livre qui se lit comme un roman policier; en d'autres termes, la véracité de l'histoire est la condition même de l'analogie littéraire. Ce n'est plus alors l'écriture mais bien la personne de l'auteur qui donne au livre sa valeur. Réciproquement, le livre suffit désormais à faire 1'« auteur " là où ce sont des dispositions incorporées qui faisaient l'écrivain. Ainsi, la dite «invasion des graphomanes » marque-t-elle le repli de ce principe génératif qu'est l'écrivain, instance de production souterraine, intime et opaque, au bénéfice de ce principe référentiel qu'est l'auteur, instance de rationalisation a posteriori, publique et transparente ${ }^{9}$.

11 Réunissant, le plus souvent, des auteurs de circonstance proprement dits, l'émission littéraire n'est le théâtre d'aucune conversion (i.e. elle ne sacre pas écrivain), le titre d'auteur n'étant qu'un nouvel attribut distinctif accusant encore la fragmentation d'un sujet voué à la dispersion ${ }^{10}$.

\section{L'auteur se livre : le principe juridico-éthique de responsabilité}

12 Loin de décrocher sur le plateau une nouvelle identité, ô combien prestigieuse de surcroît, l'invité s'expose au contraire, de par la valeur référentielle attachée à la fonction auteur, à voir remises en question les positions qu'il occupe par ailleurs, dans d'autres champs que celui de la production littéraire. C'est du moins ce qui apparaîtra au terme de la transcription d'un numéro d'Apostrophes consacré précisément à un genre privilégié de graphomanes.

Bernard Pivot - Il n'est pas rare que dans les journaux, les postes de rédacteur en chef, d'éditorialiste soient confiés à des écrivains. Rappelez-vous Mauriac, Camus et puis aujourd'hui d'Ormesson, Dutourd, etc. Et puis, il arrive aussi que ces mêmes postes soient occupés par d'anciens professeurs, d'anciens chercheurs, des gens qui à un moment donné ont été tentés par la politique. Bref, par ceux que j'appelle les « intellectuels-journalistes » [Bernard Pivot présente ensuite ses invités]. 
Jean Daniel (directeur du Nouvel Observateur) - Ma première ambition était d'être écrivain. Je voulais être un écrivain qui fasse du journalisme.

. P. - Et vous êtes plutôt devenu un journaliste qui fait des livres. Et vous, JeanFrançois Revel [directeur de L'Express], qu'est-ce qui fait que l'écrivain et le professeur sont devenus journalistes ? [...]

B. P. - Alors, Guy Hocqenghem est à Libération. Libération a annoncé très joliment l'émission d'aujourd'hui. Je lis: « Notre éminent collaborateur Guy Hocqenghem, pour son livre: La Beauté du métis, est livré aux vieilles badernes de la presse; comment va-t-il s'en tirer, comment va-t-il s'habiller [il est en jean, tee-shirt, baskets, alors que les autres sont en costume-cravate]. Alors, vous êtes professeur de philosophie à l'université de Vincennes...

G. H. - Non, je suis chargé de cours.

B. P. - Bah... Vous êtes écrivain et vous êtes journaliste à Libération; est-ce que ce sont des activités intellectuellement complémentaires?

G. H. - Bof, ça c'est des petits jobs.

B. P. - Mais imaginez que vous gagniez mieux votre vie à Libération, est-ce que vous abandonneriez votre carrière professorale?

G. H. - J'ai pas de carrière professorale.

B. P. - Georges Suffert [directeur du Point], vous c'est un peu l'itinéraire inverse : vous avez publié des essais, des pamphlets et maintenant, à cinquante ans, vous publiez une fiction. C'est parce que vous en aviez marre du ronron des idées?

G. S. - Peut-être parce que j'ai toujours eu envie d'écrire... Enfin d'écrire réellement, quoi, et pas être uniquement journaliste et que j'ai jamais osé par une espèce de respect bizarre de la littérature, qui pour moi est la chose la plus importante.

J. D. - Il n'y a pas de vraie frontière, surtout pour les reporters. Moi, j'ai fait toute ma vie du reportage et je n'ai jamais senti que la frontière avec le romanesque était étanche.

B. P. - Alors, La Fête au Togo ? Ce sont sept nouvelles que vous faites paraître aux éditions Grasset. Il m'a semblé que le dénominateur commun de vos nouvelles, c'était la recherche de quelque chose qui touche la métaphysique.

G. S. - Oui, mais on ne va pas ennuyer tout le monde avec la métaphysique.

B. P. -Oui, non mais il faut quand même en dire deux mots.

G. S. - [...] Mais je crois qu'il faut pas ennuyer les gens avec ça; les gens, ça les embête. Lui [Guy Hocqenghem], ça l'embête.

G. H. - [hors champ] Ça c'est sûr, oui !

G. S. - Donc, c'est mon affaire à moi tout seul ; j'essaye de faire des histoires avec des gens que j'ai rencontrés et qui pour moi étaient des saints; mais un saint, ça ne porte pas d'auréole sur la tête.

B. P. - Alors par exemple, ce général il est inventé ou...

G. S. - Cette histoire, elle est extraordinaire. Tout le monde me dit que c'est inouï d'avoir arrangé une histoire aussi fausse. Le seul ennui, c'est qu'elle est intégralement vraie, de la première à la dernière ligne. C'est tout ce que je peux dire.

B. P. - Et cette histoire de rotatives qu'on envoie en Afrique, qu'on implante sur un lieu sacré et qui ne marchent pas; on envoie les ingénieurs, tout est normal et pourtant ça ne marche pas.

G. S. - Je vais avoir l'air d'un ballot; on croit que j'ai inventé et pourtant elle est vraie l'histoire de la rotative.

J. D. - C'est du reportage, c'est bien ce que je disais! Moi, je n'ai pas salué la conversion de Georges Suffert comme la critique, parce que j'ai toujours vu dans ses reportages les preuves qu'il possédait l'art du récit.

B. P. - Guy Hocqenghem ; alors, vous qui vous en prenez avec une extrême violence au ton français; est-ce que vous considérez que Georges Suffert est dans le bon ton français?

G. H. - Ah, tout à fait! Moi, je pense qu'un livre comme celui de Georges Suffert, bon je vais le vexer peut-être un peu, je pense qu'il n'aurait jamais été publié si Suffert 
n'était pas éditorialiste et directeur d'un grand magazine. En fait, je pense qu'il y a un type de littérature française tout à fait typique qui est celle qui s'assure une édition par la position de pouvoir des gens. Je pense qu'il y a comme ça des faux romanciers, des faux nouvellistes, des faux écrivains, qui sont simplement des gens qui ont suffisamment de pouvoir pour se faire éditer. Et Suffert en est un cas absolument typique!

B. P. - Georges Suffert?

G. S. - C'est bien possible. Sur du journalisme, je me défendrais, mais là-dessus, pas du tout.

J. D. - Je ne serais pas aussi masochiste que Georges Suffert [rires]. D'abord parce que j'ai lu avec un grand plaisir le livre de Guy Hocqenghem, mais que je trouve que rien n'est plus classique, plus français, plus parisien que ce merveilleux petit pamphlet. Je suis un lecteur de Libération et je trouve une différence très grande entre le "style Libé » que vous avez dans vos articles, que je trouve d'ailleurs pétulant, et ce style merveilleux, tellement classique dans le bonheur de l'expression. Vous avez une cravate quand vous écrivez; vous n'êtes pas en jeans ! [gros plan sur G. H. qui jusque-là écoutait la leçon : il renifle comme un écolier pris sur le fait, et ensuite se gratte le nez].

$[\ldots .$.

J.-F. R. - J'écoutais parler Hocqenghem tout à l'heure et je me disais : c'est parfait ! Il a dix ans de retard, c'est un cliché ambulant:: Vincennes, soixante-huit... Il parle comme il faut et c'est très agréable parce que comme ça on sait où on se trouve.

G. H. - [off] Avec vous trois, on sait encore mieux !

[A propos de l'anthologie des meilleurs articles parus dans L'Express présentée par son actuel directeur Jean-François Revel, une discussion s'engage avec Georges Suffert et Jean Daniel, anciens collaborateurs tous deux de l'hebdomadaire.]

G. H. - Je trouve que vous avez été culottés tout à l'heure de dire que j'avais dix ans de retard, quand on voit où vous en êtes! Vous en êtes à vous disputer le passé de L'Express... Vous voyez, ils viennent tous les trois du même journal! Les téléspectateurs ne savent pas forcément ce genre de choses.

[Jean Daniel parle de son livre et Georges Suffert dit ensuite tout le bien qu'il en pense.]

J. D. - Vous ne me gênez pas plus par votre approbation que lui [G. H.] par sa désapprobation.

G. H. - Ce livre, c'est le livre des dîners en ville.

J. D. - Écoutez-moi, je me sens très mal à l'aise avec ce numéro d'histrion.

G. H. - D'histrion? Et votre numéro de vieux sages alors?

G. S. - Bon, je vais vous dire quelque chose : vous avez écrit un bouquin qui est très intéressant à cause des trois dernières pages.

G. H. - Oh bon, d'accord.

G. S. - Alors, ne nous donnez pas de leçons. C'est très difficile de faire un livre.

J.-F. R. - Le salonnard ici, c'est vous.

G. H. - Oh ça va, vous êtes tous les trois de la presse assise, installée, confortable et tout.

J.-F. R. - Vous nous faites un numéro classique de salonnard.

B. P. [revenant au livre en question] - Jean Daniel, alors finalement, vous dites que vous vous voyez dans la peau d'un dissident. Expliquez-nous ça : comment on peut être directeur d'un grand journal et dans la peau d'un...

G. H. [off] - C'est assez osé, en effet !

[J. D., air de compassion, en regardant Pivot.]

B. P. [à G.H.] - Laissez-le répondre.

J. D. - Ça ne m'amuse pas tellement. Je risque de manquer d'humour avec ce genre de réflexions parce qu'elles n'ont même pas la portée essentielle du dialogue qui mène à une sorte d'opposition réelle, fondamentale et complète. Rien n'est plus parisien que ce livre, ces invectives et ces discussions marginales [il s'adresse à B. P., tandis que ce dernier fait taire G. H. par des « chut » !]. Si encore il me contestait 
sur un point... j'ai passé ma vie à être contesté.

G. H. - Jean Daniel, est-ce que je peux vous poser une question sur un point précis ; je vous promets que ce n'est pas une invective.

J. D. - Je ne vous prends pas au sérieux.

G. H. - Ça m'est égal que vous ne me preniez pas au sérieux; ce n'est pas à moi que vous parlez, c'est au téléspectateur; il faut bien se mettre ça dans la tête.

Les trois, en chœur - On est tout à fait d'accord ; votre numéro est tout à fait pour les téléspectateurs.

G. H. - Le nom que vous donnez à vos aventures: L'Aventure du vrai [titre de l'anthologie présentée par Jean-François Revel], L'Ère des ruptures [titre de l'ouvrage de Jean Daniel], c'est un type de proclamation idéologique qui me paraît extrêmement hypocrite, c'est vrai, parce que ce sont des journaux à nature profondément commerciale...

J. D. - Bon, alors moi qui voulais vous ménager... Si c'est de l'hypocrisie, alors ça [le livre de G. H.] c'est de l'imposture ; et l'imposture, ça consiste à prendre les parures de la marginalité pour rentrer dans le pamphlet le plus conformiste, le plus classique qui soit. Vous savez, pour être iconoclaste, ce que vous prétendez être, il faudrait qu'il y ait des icônes ; mais mon pauvre, il n'y en a plus des icônes! Vous avez une grand-mère juive? J'en ai deux! Vous avez été sur le terrain? J'ai été partout! Qu'est-ce que j'ai à envier de vous?

G. H. - Vous êtes directeur d'un grand journal, plein de publicité, riche et puissant, et vous êtes près du pouvoir.

B. P. - Venons-en à votre livre Guy Hocqenghem. Vous dites votre haine de la France, que vous écrivez avec un petit «f $\mathrm{f}$, vous dites votre supplice d'être né français et en même temps vous chantez La Beauté du métis [titre de son ouvrage]. Alors, est-ce que c'est une thèse très insolente pour faire effectivement un livre provocateur, ou bien est-ce que très profondément vous ressentez cette haine contre la « france »?

G. H. - Écoutez, ce n'est pas une thèse... C'est très curieux, vous êtes en train de me demander si je mens ou si je dis la vérité. Alors, qu'est-ce que vous voulez que je vous dise : qu'effectivement j'ai fait un livre comme dit Jean Daniel pour être dans un salon où je suis pour l'instant!

J.-F. R. et J. D. [commentaires en voix off - Vous êtes bien susceptible !

B. P. - Non, mais il arrive parfois que pamphlétaire, on se laisse emporter par une violence... vous dites des choses très dures contre la France.

G. H. - Je pense qu'on est en train d'en avoir un bon exemple avec cette émission. Ca s'appelle les « intellectuels-journalistes » par exemple ; je reviens un peu là-dessus : ça me paraît très français cette appellation pour désigner tout simplement ceux qui ont le pouvoir dans les trois principaux hebdomadaires d'information français.

B. P. - Mais vous êtes un intellectuel, vous aussi !

G. H. - Oui, sauf que j'ai pas de pouvoir; je suis pas dans un grand hebdomadaire, etc.

J.-F. R. - Ben, vous avez le pouvoir vous aussi : vous parlez à la télé !

G. H. - Oh, arrêtez! Vous parlez toutes les semaines dans votre hebdomadaire ; pour une fois que quelqu'un parle un peu différemment !

J. D. [off] - Il dit que l'émission est un spectacle et il en est le meilleur acteur.

G. H. - ...Tout le monde ici s'exprime comme si c'était des décideurs; et ce sont des décideurs. Les vrais intellectuels, c'est vous, dans la mesure où vous décidez effectivement ce que doit être la tendance intellectuelle du moment. C'est révélateur dans votre livre, Jean Daniel, tout ce que vous dites sur Soljenitsyne, sur la façon dont a commencé ici la dénonciation du goulag : vous en êtes pratiquement l'inventeur.

B. P. - Mais enfin si je vous ai invité, c'est précisément parce que vous étiez représentatif d'un autre journal, d'une autre génération et d'un autre courant d'opinion. Alors vous avez la parole, là : vous pouvez vous exprimer...

G. H. - Ben, je m'exprime. 
B. P. - Vous aussi vous avez un quart de ce pouvoir de parler à l'antenne; alors parlez-nous un peu de votre livre.

J. R. [off] - Mais oui, vous perdez votre temps à parler de nous; parlez de votre livre!

J. D. - Sauf qu'il y a une chose.

B. P. - Non, non, laissez-le parler.

J. D. - Sauf qu'il y a une chose : il faut qu'il soit solitaire, qu'il ignore nos divisions entre nous; et pour se poser dans une originalité particulière, comme image de marque, la solitude est une chose importante. J'aurais beau lui dire que je suis d'accord avec telle ou telle chose... d'ailleurs, je vais vous compromettre de cette manière tout à l'heure [rires].

B. P. - Vous n'en aurez pas le temps... [à G. H.] - Venons-en à votre livre : pourquoi cette haine de la France?

[G. H. expose son point de vue et prend comme exemple opposé celui du noir américain qui « joue un rôle culturellement important ».]

J. D. [off]-C'est évident.

G. H. - ... alors qu'en France, il n'existe aucune mixité, aucune immixtion culturelle de l'arabité ; il n'existe aucun métissage culturel.

J. D. - Question : y a-t-il un métissage aux États-Unis?

G. H. - Oui.

J. D. - Un métissage?

G. H. - Enfin, les États-Unis sont un melting-pot culturel ; c'est pas une nouveauté !

J. D. - Non. C'est une mosaïque de communautés juxtaposées. Alors là, je m'excuse ! New York est un melting-pot? Vous connaissez des mariages mixtes? [il s'adresse là à J.-F. R. qui avait manifesté sa désapprobation; G. H. est saisi en gros plan dans l'attitude de celui qui est mis sur la touche].

J.-F. R. - Si, de plus en plus.

J. D. - Mais jamais de la vie ! Dans certaines sociétés artistiques... Mais, je suis tout à fait d'accord là-dessus avec Guy Hocqenghem...

G. H. - Ben, si vous êtes d'accord alors... J. D. - ... sur la forme.

J.-F. R. - Moi, je suis d'autant plus d'accord que j'ai écrit un livre pour raconter ça, qui a été publié en 1970, qui s'appelait : Ni Marx, ni Jésus, qui l'expliquait en long et en large.

G. H. - D'accord, chacun a écrit un livre qui expliquait tout ça avant ; mais c'est très bien!

J.-F. R. - Non, mais écoutez, si vous n'avez lu que votre propre livre, ce n'est pas de ma faute. $Y$ en a eu d'autres avant?

B. P. [à G. H.] - Autre raison de haïr la France?

G. H. - Ça, c'est la première raison. La seconde, c'est que l'horizon culturel est dominé par l'opinion, le commentaire. C'est très curieux, en France le rôle des intellectuels, de gauche en particulier, qui est sans cesse de commenter ce qui se passe dans le monde. Ce sont des donneurs de leçon permanents et ça, ça me paraît être une caractéristique culturelle française !

B. P. - Mais vous donnez une sacrée leçon là, dans votre livre.

[Rires - J.-F. R., narquois, se retourne vers G. H.]

J. D. - Pour parler de manière plus sereine de ce livre, qui est très bien écrit, encore une fois, dans la langue la plus heureuse, la plus classique et la plus bourgeoise qui soit, mais très bien écrit... [On voit G. S. sourire et hocher la tête, comme pour dire : là, vraiment, il en fait trop ! On entend J.-F. R. glousser.]

J. D. - C'est vrai, je l'avoue, j'ai tort.

G. H. - Y a qu'un seul français, c'est un des problèmes.

J. D. - Non, puisque vous en employez un autre dans Libé, et il est très heureux aussi.

G. H. - Non, c'est le même !

J. D. - Si vous permettez alors, pour parler du fond du problème et pas de vous, pour parler de votre livre avec sérénité, je trouve qu'il y a toute une partie où je me suis senti représenté, exprimé. C'était pas nouveau, mais se sentir exprimé une nouvelle fois, c'est bien, et quand c'est dans cette langue, c'est tout à fait un 
plaisir... Le métissage, l'éloge de la bâtardise, ça a été une partie de ma vie. J'ai toujours pensé ça... Ajouté à une certaine influence gidienne qui donne une sorte de nostalgie de cette mixité. Enfin, le caractère chauvin et xénophobe d'une grande partie de la France est évident.

G. H. - Il suffit d'assister à un contrôle d'identité dans le métro, qui est contrôlé ? les étrangers.

J. D. [off] - Pas seulement!

G. H. - Ou nous en tant que possiblement étrangers... Enfin moi, pas vous !

J. D. - Vous ! Et là où il a encore raison, c'est que... et c'est pour ça que je voulais l'y conduire... c'est que contrairement aux États-Unis, le seuil d'intolérance n'est pas du tout atteint en France. [G. H. essaie d'intervenir.] Alors, pour ne pas finir sur une approbation sans réticences, qu'est-ce qui m'a gêné ? [G. S. et J.-F. R. ricanent.] Eh bien, c'est que vous glissez : il vous fallait une icône dure, les molles avaient déjà été détruites. La dure, c'est la littérature. Vous avez fait le couplet fameux de la littérature classique camisolée par Malherbe, Boileau, à laquelle on peut vous opposer Rabelais, Sade, Céline, Lautréamont. Ça, c'est vrai. La seule charge que vous oubliez, c'est que la littérature française est probablement, quand même, notre porte sur l'extérieur. [Gros plan sur G. H. qui ouvre la bouche pour parler.] Pour rester sur votre terrain, Dostoïevski, il s'est emparé de Balzac. Est-ce que vous savez, ou plutôt est-ce que vous vous rappelez, parce que vous le savez bien sûr, que Stendhal n'était pas connu quand Nietzsche en a fait une sorte de folie personnelle?

G. H. - Non, je pense que...

J. D. - ... Et Diderot, qui l'a pris, sinon Goethe et Schiller? La littérature a été pendant des siècles une ouverture. Là-dessus, je crois que vous avez fait une erreur. G. H. - Vous reconnaîtrez avec moi une chose, c'est qu'on peut tout de même, pour simplifier extrêmement, dire qu'il existe une spécificité française [s'adresse à J. D.]. J. D. [off] - Certainement.

G. H. - Certainement que la Chine a vécu quelque chose de ce genre en se considérant comme le centre du monde.

J. D. [off] - Bien sûr.

G. H. - Mais tous les conflits mondiaux sont...

J. D. - Hexagonalisés?

G. H. - Sont hexagonalisés.

J. D. - Oui, je suis d'accord.

G. H. - Et, c'est très contemporain.

J. D. - Mais, ça n'est pas nouveau.

G. H. - Je ne cherche pas à faire du nouveau.

J. D. [off] - Ben y vaudrait mieux !

G. H. - Je cherche à mettre en avant quelque chose qui n'est pas connu en France. La France se tait sur sa propre fermeture et je...

J. D. - Bernard Pivot, puisque vous me dites que c'est la fin, il y a une chose que je voudrais vous dire.

G. H. - Je reviens à Libération, attendez, je...

B. P. [à G. H.] - Non, non, attendez !

G. H. - Pfff !

J. D. - Mon livre sur lequel vous m'invitez...

G. H. [résigné] - Finalement, c'est lui qui a parlé de mon livre ; bon, d'accord !

J. D. [en passant] - Ça vaut peut-être mieux.

G. H. - Oui d'accord, bon, très bien.

J. D. - Il y avait... Mon ambition secrète dans ce livre, qui compte un peu pour moi, c'était une réflexion sous-jacente sur la violence. Et je regrette qu'ici, il n'était de violence que verbale et frivole [en regardant Bernard Pivot].

B. P. - Y a eu une violence, un peu... Bon, un des personnages de Georges Suffert dit : il n'y a qu'une chasse royale, c'est celle de la vérité. Est-ce que vous faites vôtre cette phrase [à J. D.] ? 
J. D.-Oui, sauf que je n'aime pas employer des mots comme vérité.

B. P. - Vous non plus [à J.-F. R.].

J.-F. R. - Comme je l'explique dans la préface : dans le domaine de l'information, le seul garde-fou c'est la diversité ; il faut préserver la diversité.

B. P. [faisant l'impasse sur G. H.] - Ben aujourd'hui, y a eu quand même de la diversité ici...!

J.-F. R.- Oh oui ! [sourire entendu avec B. P.].

G. H. [off] - Pfff! ${ }^{11}$ catégories dichotomiques d' "auteur » et d'«écrivain » implicitement attribuées à chacun, émergent ici du débat lui-même (elles ne lui préexistent pas), dont le caractère polémique vise, qui plus est, directement la question de la vérité référentielle. D'autre part, l'attribution implicite de telle ou telle catégorie est indépendante de la qualité du livre. Elle sanctionne plutôt le type de rapport qu'entretiennent l'identité sociale que les uns et les autres affichent sur le plateau (ici : les positions occupées dans le champ journalistique) et leur personnalité que révèle la confrontation de chacun au principe de sincérité (ici: les uns se révèlent «hypocrites» et l'autre "imposteur»). La dramaturgie du débat s'organise en effet sur la base d'une première dénégation: «Georges Suffert n'est pas un écrivain », en appelant réciproquement une autre : «Guy Hocqenghem n'est pas un auteur ». D'un côté, Georges Suffert qui aspirait avec humilité à devenir crivain, se portait volontaire pour la grande conversion, est remis à sa place par l'approbation insidieuse de Jean Daniel dont la célébration du reportage réduit à néant l'écart entre écriture et journalisme; tandis que Guy Hocqenghem, plus radicalement encore, ramène l'écrivain à l'auteur en rapportant la publication de l'œuvre à la seule position de pouvoir qu'occupe Georges Suffert. A l'inverse, Guy Hocqenghem s'avère d'emblée dans l'incapacité d'assumer le rôle de l'auteur, en s'opposant d'abord à toute tentative de lui assigner une position, ensuite en ne parlant pas de son livre; tandis que la même approbation insidieuse de Jean Daniel vient le discréditer comme auteur en félicitant l'écrivain.

\section{L'author's studio}

\section{L'auteur est un acteur}

A propos d'un numéro d'Apostrophes en partie consacré aux mémoires de la star hollywoodienne Kirk Douglas, Alain Rémond s'interroge dans sa rubrique hebdomadaire de Télérama: "Qui rafla la mise et fit la différence, en trois coups de cuillère à pot, entre les acteurs amateurs que sont les écrivains, sur un plateau de télévision, et un vrai de vrai acteur professionnel, écrivain d'occasion? Et, qu'est-ce qui est le mieux finalement chez $\operatorname{Pivot}^{12}$ ? » Pour les téléspectateurs de cette émission, la réponse ne fait aucun doute, non plus d'ailleurs que pour quiconque, puisque aussi bien, poser la question c'est déjà y répondre. Simple effet rhétorique, cette interrogation retient cependant notre attention pour ce lieu commun qui lui sert de présupposé : les écrivains sur un plateau sont des acteurs. La métaphore théâtrale pourrait effectivement constituer un prolongement à cette thématique de l'auteur que l'on vient de quitter, à condition toutefois d'en préciser les modalités d'utilisation et d'en apprécier les conditions de validité. Incontournable, puisque aussi bien elle est convoquée dans cette même rubrique de Télérama chaque fois qu'il est question de la «dramatique du vendredi soir", la métaphore théâtrale s'avère au demeurant 
singulièrement féconde, en cela qu'elle permet de dessiner une véritable galerie des portraits d'auteurs en acteurs. C'est comme si Apostrophes ne valait que par les numéros d'acteurs de ses invités, comme si chaque émission ne valait que d'en élargir encore le répertoire. Après le grand acteur, la « star », voici donc venir la «starlette ».

Irène Frain est une charmante personne. Elle a de jolies jambes, de jolis genoux, de jolis yeux. Écrit-elle joliment? Je n'en ai, hélas, pas la moindre idée, n'ayant à ce jour pas lu une seule ligne de sa littérature. Tout ce que je peux dire, c'est qu'elle est joliment casse-pieds.

A Apostrophes, on n'a vu, on n'a entendu qu'elle. Pour commencer, à l'en croire, ce brave Anthony Burgess, assis à ses côtés, était tombé fou amoureux d'elle. «Si je ne m'abuse, vous avez, n'est-ce pas, cher maître, minaudait-elle, immédiatement reconnu en moi cet instrument de musique qu'est, à vous lire, la femme que vous appelez de vos vœux et dont à laquelle d'ailleurs et en effet... » Fourrageant dans sa tignasse, Burgess grommelait des borborygmes et lâchait des interjections, à tout hasard et sans trop se compromettre.

Puis Irène Frain, au fil de l'émission, tint à assurer de son admiration, de sa sollicitude, de son intime compréhension du noyau le plus secret de leur œuvre tous les auteurs présents sur le plateau. Façon de faire comprendre qu'elle était de leur trempe et qu'elle n'en attendait pas moins d'eux à son endroit.

Enfin, ce fut à elle. Je veux dire, à son livre à elle. Pivot eut à peine le temps de poser une question qu'elle se lança dans une vibrante, enthousiaste et, surtout, interminable défense et illustration de son propre univers, de son style, de ses images, de son propos, de sa singularité... Pivot avait beau lever le doigt, pour humblement demander la parole, comme à l'école, impossible d'en placer une.

La physiologie, heureusement, a ses lois et Irène Frain, un court instant, dut s'interrompre pour reprendre son souffle. Pivot, piaffant dans les starting-blocks, s'engouffra dans la brèche: "Il se trouve, chère madame, que Bertrand PoirotDelpech, ici présent, vous a, dans son feuilleton du Monde, et pas plus tard qu'hier, quelque peu éreintée, n'est-ce pas, Poirot-Delpech ?» Funeste erreur ! Irène Frain saisit la balle au bond : figurez-vous qu'elle aussi l'avait lu, l'article de ce « monsieur Poirot » (comme elle ne cessa de l'appeler) et qu'il n'était pas du tout négatif, et qui donc plus qu'elle pouvait en juger, puisque son univers, son style, ses images, son propos, sa singularité à elle, «n'est-ce pas, monsieur Poirot », et d'ailleurs l'article soulignait très bien que par rapport et nonobstant et sans oublier cet autre aspect fascinant, et cette façon si personnelle, si originale, «que vous avez si bien sentie, monsieur Poirot... »

Pivot, miracle, réussit l'exploit du siècle: intercepter le ballon et relancer en direction de Poirot-Delpech. «Mais enfin, j'ai bien lu votre article, vous êtes plutôt ironique... » On vit alors ce singulier spectacle : hypnotisé en direct (je ne vois pas d'autre explication) par le discours (et les yeux?) de l'intarissable Irène, PoirotDelpech fit l'étonné : «Moi? vraiment? Ironique? Vous croyez? Franchement, ça m'étonnerait... Non, pas du tout, au contraire, son livre, je le trouve, comment dire...ou plutôt...et même à la réflexion, assez...non, sincèrement...ce n'est pas ce que j'ai voulu dire...son style, ses personnages...son univers...» Irène Frain, extatique, buvait du petit lait. Ce qui, du coup (et à mon grand soulagement) la rendit muette. Pivot, épaté, diagnostiqua, au débotté, un cas de "schizophrénie entre le critique dans son bureau et l'auteur sur un plateau ». Il n'y avait pas de psychiatre dans le studio. On passa à autre chose ${ }^{13}$.

Pareille insistance dans l'usage de la métaphore théâtrale se conçoit aisément : elle est cela et cela seulement qui permet au critique de télévision de rendre compte d'une émission littéraire. A telle enseigne que c'est encore par rapport à la figure repoussoir de l'acteur que peut se dessiner une excellence de l'écrivain; la représentation le cède alors à la présence. 
Le Clézio, en plus de son look Sam Shepard, c'est une force en-dedans, une porte qui s'entrouvre sur des mystères, un royaume souterrain. Quand il parle, c'est presque à contrecœur, les yeux baissés, en butant sur les mots, laborieusement, et on voit qu'il cherche, qu'il ne veut pas tomber dans le n'importe quoi. Alors qu'il pourrait! Une star, ça peut balancer deux, trois clichés, l'air entendu, avec un petit clin d'œil, et hop, tout le monde est content. Le Clézio, lui, il a l'air tout humble devant ce qu'il a envie de dire, qui le dépasse, et qu'il veut pourtant transmettre. La présence aux choses, l'unité des origines, le grand rêve oublié... Alors il s'applique, comme en s'excusant de nous embêter avec tout ça. Là, à la télé, lieu de babil aboli dans l'instant. Et, c'est forcément injuste pour les autres : Tom Wolfe, du coup, avec son costard Gatsby, ses chaussettes à pois et ses souliers bicolores, il a l'air en représentation, il fait faux, toc. Et même s'il nous fait rire avec sa folle vision de New York, après l'intensité de Le Clézio, ça fait second choix. Ça fait télé. Sur tout ce qu'il dit, sur son image, il y a en surimpression, la présence de Le Clézio, qui finit par effacer tout le reste. Que faire contre une star ${ }^{14}$ ?

On l'aura constaté, au vu des précédents extraits, longuement reproduits: l'omniprésence de la figure de l'acteur n'a d'égal que l'évidence de sa convocation. Or, ce sont précisément les conditions tenues pour acquises d'une telle évidence que nous entendons considérer désormais. En nous rapportant, à notre tour, à telle fameuse émission ayant réuni autour de Kirk Douglas, Philippe Sollers, écrivain au faîte de la gloire médiatique, un autre écrivain, d'origine russe, V. Volkoff, Jérôme Garcin, responsables d'un Dictionnaire des écrivains et le célèbre publiciste Jacques Séguéla pour un ouvrage sur les stars ${ }^{15}$.

B. P. - Alors maintenant je vais demander à Jacques Séguéla qui est notre « expert » en stars, s'il va oser répéter ce qu'il a dit au micro de Jean-Pierre Elkabach ce matin [rumeurs sur le plateau], à savoir que Kirk Douglas n'était pas une star ! Je dois dire que j'ai failli tomber de ma chaise quand j'ai entendu ça! Voulez-vous répéter ça devant l'intéressé...

J. S. - Mais bien sûr ! Et je crois qu'il va partager d'ailleurs mon analyse... C'est un immense acteur... évidemment.

B. P. [ironique] - Ah bon!

J. S.-Et d'ailleurs le mot star aux États-Unis n'a pas la même signification qu'en France [sic!] donc il faut bien qu'il comprenne les choses. En fait pour moi une star c'est un porte valeur, à la différence d'ailleurs d'une vedette qui est simplement une grande célébrité. La star en plus, à un moment donné, illustre une valeur. Brigitte Bardot c'était la libération de la femme, par exemple John Wayne a illustré le bon américain et Clark Gable la beauté de l'Amérique. Mais lui a choisi la colère (gros plan sur Kirk Douglas, impassible) et la colère n'est pas une valeur. Toute sa vie a été concentrée sur la colère, parfois la violence, parfois la méchanceté... Il dit «je préfère être le méchant parce que le méchant est photogénique » [gros plan sur Kirk Douglas qui approuve]. Il a d'ailleurs tout à fait réussi. Une star est finalement le miroir de nos fantasmes, qui nous ouvre des petites fenêtres vers l'imaginaire. Pas Kirk Douglas. Dans un premier temps de sa carrière, comme tous les jeunes premiers, il a fait rêver toutes les jeunes minettes du monde; mais dans le second temps de sa carrière... ce qui reste de lui, c'est l'immense acteur.

B. P. - Vous avez vu ce qui reste de lui ! [désigne Kirk Douglas]. C'est pas mal hein! Non mais vous avez vu ce qu'il reste de lui ! [Rumeurs d'approbation dans la salle pendant que Kirk Douglas se prend la tête dans les mains.]

J. S. - Oui, oui... Mais mieux vaut être un immense acteur qu'une star. Le destin des stars est horrible. J'ai d'ailleurs lu dans une interview qu'il a donnée il y a deux ans ou trois ans que finalement John Wayne n'était pas un grand acteur, il faisait en permanence du John Wayne. Il dit même : «Moi je ne suis pas une star, je suis un acteur, et j'essaye d'être un grand acteur. »

B. P. - Oh! Non, non, l'un des chapitres de son livre s'intitule « Star ». 
K. D. - Je voudrais savoir comment vous avez décidé d'être l'homme qui décide qui est une star, qui n'est pas une star. [Rires.]

Philippe Sollers - Si je peux dire un mot, si je peux dire un mot...Moi je crois que si une star c'est quelqu'un qui se prend pour son image, qui s'identifie avec son image, alors Kirk Douglas n'est pas une star. Il ne se prend pas pour Kirk Douglas, ce qui lui donne des qualités humaines de profondeur tout à fait extraordinaires. Ça c'est une valeur. La seconde valeur, elle est évidente et je m'étonne que vous ne l'ayez pas remarquée (à Jacques Séguéla) : c'est que c'est quelqu'un qui est fidèle à ses origines et qui étant donné son parcours, reste je crois distinct de tout le monde du spectacle, de toutes les images parce qu'il est fidèle, parce qu'il est juif et que cette valeur là, il la dit tout le temps avec beaucoup de fermeté [pendant toute la durée de la tirade, son bras tendu désigne Kirk Douglas].

K. D. - Monsieur Pivot [les bras levés], j'ai peur, j'ai peur. B. P. - Non, pourquoi ?

K. D. - Je crois que dans cette émission, ce soir, je vais découvrir que je ne suis pas une star [rires].

B. P. - Mais vous le savez bien que vous êtes une star.

Vladimir Volkoff - Et que votre seul mérite c'est d'être juif [Kirk Douglas éclate de rire]. C'est la seule conclusion.

K. D. [en réponse à V. V] - Ou peut être je vais devenir écrivain !

J. S. - ...Il n'est pas star dans la définition que j'ai voulu donner des stars qui d'ailleurs, remarquez-le, n'ont pas eu de vie exemplaire...

B. P. - Non mais écoutez, moi je vais vous dire: pour moi c'est une star tout simplement parce que c'est un chiffonnier, il est devenu un des plus grands acteurs $\mathrm{du}$ vingtième siècle, il a tourné dans des films qui sont des chefs d'œuvre [il s'enflamme sous le regard bienveillant de Kirk Douglas].

P. S. - Ben voilà !

B.P.-Et je... Non laissez-moi finir (à J. S.), il est en France depuis quelques jours et tous les journaux se sont arrachés Kirk Douglas, toutes les télévisions voulaient l'avoir... C'est ça une star!

K. D. - Non, non, non Monsieur Pivot, vous n'avez pas raison ; c'est parce que je suis un écrivain ! [Rires, applaudissements.]

[...]

B. P. - Vladimir Volkoff, qu'en pensez-vous? V. V. - Du livre de Kirk Douglas ? B. P. Non, non de Kirk Douglas.

[V. V. souligne l'engagement de Kirk Douglas pour la cause des personnes agées, P. S. surenchérit en mentionnant son attitude courageuse pendant le maccarthysme.] K. D. - Je ne suis pas une star, je suis un héros ! [Rires]

B. P. - Alors maintenant c'est à vous Philippe Solers. Évidemment, c'est pas un cadeau de passer derrière Kirk Douglas, hein.

P. S. - Si ! [tend le bras vers Kirk Douglas cherchant son assentiment; il présente ensuite brièvement son livre]... je crois qu'un écrivain aujourd'hui ne subsiste dans le monde du spectacle généralisé où nous sommes et où quelques personnes seulement sont lucides, enfin Kirk Douglas l'est, qu'avec beaucoup de malentendus. Un écrivain dans notre société n'est pas très à l'aise, pourquoi ? Parce qu'elle est spectaculaire...

B. P. - Bien sûr!

P. S. - Et qu'il faut aller vite, et qu'on ne lit pas vraiment les livres...

B. P. - Ben écoutez-moi je vous ai lu attentivement.

P. S. - Merci.

B.P.-Et je vous ferai remarquer par exemple que vous cultivez à plaisir les malentendus. Page 108, vous notez...

$[\cdots]$

B. P. - Garcin, votre jugement ! J. G. - Sur..

B. P. - Sollers... le roman.

J. G. - Sollers, ah ! C'est une « star » Sollers !

B. P. - Ah oui ! A propos est-ce que c'est une star Sollers ? [à J. S.] 
J. G. - C'est la dern....

J. S. [en même temps]-C'est certainement une star de l'avenir.

J. G. [agacé] - Mais non, mais non!

B. P [off] - Ça fait trente ans qu'il est célèbre!

J. G. - Alors ça ! On peut se battre sur Sollers...

J. S.-Nous sommes dans une époque médiatique qui a beaucoup occulté les stars de

l'écrit pour laisser la place aux stars du business, du sport..

J. G. - Sollers est la dernière star... le dernier représentant de la vie littéraire avec ce qu'elle a de fou, parfois d'énervant, de superbe!

B. P. - C'est pour ça d'ailleurs que je l'ai invité. J. G. - Mais je sais bien!

En fin d'émission, Bernard Pivot présente brièvement d'autres ouvrages, parmi

lesquels un livre consacré à Jacques Séguéla.

B. P-... et puis à propos de Jacques Séguéla, parce que Séguéla c'est une star...

J. S. - Mais non justement. S'il y en a un justement qui n'est pas une star, c'est moi.

B. P. - Mais qui peut se vanter d'avoir un livre sur lui ?

J. S. - Mais non, ne le laissez pas repartir en pensant que je suis une star et pas lui [à

propos de Kirk Douglas].

Ici encore, la querelle sur le statut respectif des invités («star » ou pas «star ») occupe le devant de la scène; elle est même le thème central de l'émission. Elle se résout néanmoins dans une identité entre écrivain et acteur, identité d'autant plus emblématique qu'elle s'opère par un alignement sur cette figure imposée par la médiatisation qu'est la «star» ("star» de l'écran, "star» de l'écrit). Plus que de confusion des genres, c'est d'un jeu avec le «malentendu» qu'il s'agit. D'un côté l'acteur (celui-là même dont la carrière est déjà marquée par son interprétation du rôle d'un peintre visionnaire - Van Gogh), joue ostensiblement à faire comme s'il était écrivain. Tandis que l'écrivain véritable (tout au moins celui qu'institue comme tel sa seule présence sur le plateau) reconnaît devoir aux seuls malentendus que lui-même entretient, en jouant par exemple à faire l'écrivain (c'est-à-dire en collant littéralement à l'image de l'écrivain), de demeurer écrivain dans notre société du spectacle, de l'image.

Exemplaire, cette émission l'est sans aucun doute; non cependant qu'elle établisse la supériorité de l'acteur sur l'écrivain. De notre point de vue, c'est au contraire leur identité qu'elle manifeste de manière emblématique.

Par conséquent, la question pertinente devient la suivante : quels sont les présupposés d'une telle identité? Question qui peut se reformuler de façon inversée: quelle représentation de l'écriture valide implicitement pareille identité?

\section{L'auteur : un homme-livre ${ }^{16 .}$ La norme expressive de sincérité}

20 En premier lieu, l'identité de l'auteur et de l'acteur repose sur l'illusion d'une contemporanéité entre l'expression écrite (l'écriture dans le livre) et l'expression « corporelle » (la performance sur le plateau). Or on a vu déjà comment, au moyen de différents dispositifs formels, ce dilemme (temporel) peut se résoudre dans le recouvrement (spatial) du plateau et du livre. L'identité auteur-acteur est alors la simple implication de cette autre identité entre la scène du plateau et l'espace du livre.

21 La métaphore théâtrale présuppose, en second lieu, que l'œuvre ressemble à l'auteur, qu'il y a adéquation entre manière d'être, de parler, de se tenir et manière d'écrire. Là se tient précisément le principe même d'une émission comme Apostrophes : c'est le livre qui est invité mais c'est l'auteur qui doit faire le spectacle! Quelque paradoxale que 
puisse paraître la formule, elle offre néammoins des garanties morales que Bernard Pivot ne manque pas de souligner: "Ce ne sont pas toujours ceux qui sont les plus beaux, les plus prolixes ou qui ont le plus de culot, qui passent le mieux à la télévision. J'ai vu des auteurs exagérément timides, parlant mal et qui passaient admirablement. Pourquoi? Parce que le public sentait en eux une personnalité mal à l'aise mais authentique. Et un véritable talent d'écrivain ${ }^{17}$.» Ici pourtant, le paradoxe semble redoubler pour peu, du moins, que l'on confronte ce témoignage à celui d'H. Bianciotti, auteur "apostrophé » à plusieurs reprises et répondant à ce titre aux questions de Jérôme Garcin.

Jérôme Garcin - Est-ce qu'en dix minutes on peut vraiment parler de soi et de son livre?

H. Bianciotti - Je crois qu'une minute (sur dix) suffit, car il ne s'agit que d'émotion. Il ne faut pas raconter le livre. Il faut faire passer de l'émotion, sur n'importe quoi ${ }^{18}$.

Comment les téléspectateurs pourraient-ils donc reconnaître les écrivains véritables sans même que les invités aient à parler de leur livre? Rien n'est plus simple en définitive, étant donné qu' « à la télévision, on lit à livre ouvert sur les visages ${ }^{19}$ »! Voilà qui fonde sur un principe moral de sincérité, ancré à même le visage de l'auteur, le régime de signification propre à l'émission littéraire. Ce qui ajoute une réciproque à la relation référentielle déjà notée: le livre c'est l'auteur disions-nous; et voici que l'auteur n'est autre qu'un livre! Autrement dit, la même métaphore qui est au principe de l'émission littéraire, parce qu'elle la fonde dans l'immédiateté du renvoi entre le livre et l'auteur, en manifeste l'incontournable limite: l'écriture n'y a pas de place. Insistons sur ce point : c'est un seul et unique mouvement qui, d'une part, détache l'émission littéraire de sa référence textuelle, et d'autre part, exhausse la personne de l'auteur devenu personnage de fiction emblématique de sa propre œuvre. Les termes utilisés par PPDA, pour clore une séquence de plateau d'Ex-libris, sont sur ce point exemplaires.

PPDA - Je pense que vous avez beaucoup séduit les téléspectateurs : s'ils veulent vous retrouver, c'est dans Ma vie pour un rêve, et c'est François Varigas ${ }^{20}$.

23 Au terme du processus de «fictionnalisation » de l'auteur qui s'engage alors, l'auteur sur le plateau se confond avec les personnages de ses livres. A cet égard, la prestation hautement éthylique du romancier américain Charles Bukovski figure dans les annales de l'émission Apostrophes ${ }^{21}$. Tout aussi édifiante fut celle, pour le moins belliqueuse, réalisée sur le plateau d'Ex-libris, par un certain Cizia Zykë, aventurier scrupuleux et « auteur » à ses heures.

PPDA - Voici mon premier invité dont vous connaissez certainement déjà la tête ; il s'appelle Cizia Zykë. Si vous connaissez sa tête, c'est que vous l'avez découvert à la télévision avec tout d'abord Oro, puis Sahara et enfin Paradis (il les présente à la caméra). A chaque fois, il s'agissait de ses propres aventures; et voilà qu'il se lance à son tour dans la fiction en commençant une collection avec un héros qui s'appelle Tuan Charly. Premier volume de cette collection: "Maléfices ». Alors, c'est une collection de poche, une collection de gare hein, disons, qui ne coûte pas cher. Si vous vous lancez là-dedans, c'est que vous pensez qu'il y avait un créneau à prendre, que Gérard de Villiers n'avait pas suffisamment couvert le terrain.

C. Z. [(blouson en cuir, chemise ouverte sur la poitrine, épaules qui débordent du cadre de l'écran] - Non, c'est pas du tout ça! C'est surtout que j'attaque le "monopole de Villiers"; un monopole qui n'a pas lieu d'être. Il n'y a plus de qualité, plus d'auteurs, et je n'aime pas le personnage.

PPDA - Ah bon, alors c'est une déclaration de guerre... C. Z. - Tout à fait !

PPDA - Et il faut dire que quand vous déclarez la guerre, ça se termine mal, parfois 
dans le sang. On peut parler de vous, quand même, pour que les téléspectateurs aient tout de suite en mémoire ce qu'a été votre passé. Alors, vous, vous êtes né en fait au Maroc, il y a quarante ans, d'un père d'origine albanaise, d'où votre nom un peu curieux, d'une mère grecque. Vous vivez à Minorque depuis deux mois et dans quelques mois vous vivrez sûrement ailleurs, parce que...

C. Z. - Dans deux ans, c'est-à-dire quand le pari sera gagné.

PPDA - Ah bon. Quand vous aurez écrit suffisamment de livres pour terrasser l'archange de Villiers.

C. Z. - Quand j'aurais ouvert la brèche dans le monopole. Mon intention est de publier des auteurs ; il n'y a plus d'auteurs ; un, tous les mois, à partir de 90.

PPDA - Gérard de Villiers, atttention à vos yeux, cet homme est dangereux! Vous dites vous-même que vous avez à peu près tout fait dans la vie. Vous avez tué...

C. Z. - Non, non, non.

PPDA - Vous n'avez pas tué ? Même pour votre survie?

C. Z. - Même pour ma survie ! J'aurais pu le faire, je ne l'ai pas fait.

PPDA-Qu'est ce que vous avez comme vice quand même ? Vous en avez un paquet si je lis bien vos livres! Le jeu...

C. Z. - Oui.

PPDA - Qu'est-ce que vous faites de votre argent? J'ai lu que vous aviez offert une maison à votre maman pour votre premier livre. Et après, qu'est ce que vous en avez fait?

C. Z. - J'ai aidé des amis qui sont réapparus. PPDA - On peut savoir quel genre d'amis ? C. Z. - Des amis d'aventure.

PPDA - Quel est votre sens de la morale à vous? Est-ce qu'il y a des lignes qu'il ne faut pas franchir?

C. Z.-Je n'embête personne et je me défends si on m'attaque.

PPDA - Vous avez dû embêter du monde parce que vous êtes quand même indésirable dans un certain nombre de pays. Vous avez fait du trafic. Trafic d'or, vous l'avez reconnu vous-même.

C. Z. - Oui.

PPDA - Peut-être d'autres trafics aussi ? C. Z. - Art, devises, c'est tout. PPDA - Armes, jamais?

C. Z. - Non.

PPDA - Drogue, non plus ? C. Z. - Non plus.

PPDA - Ça, c'est des choses auxquelles il ne faut pas toucher, ou ça ne s'est pas trouvé?

C. Z. - Non, la drogue, je l'utilise depuis vingt-deux ans, mais je ne la recommande pas.

PPDA - Qu'est ce qui est essentiel pour vous? Si j'en juge par vos livres, vous avez l'air d'être très loyal.

C. Z. - ...

PPDA - Qu'est ce que aimeriez faire une fois que votre collection sera lancée, que vous aurez détruit Gérard de Villiers, si tant est que vous y arriviez, parce que c'est une sacrée forteresse.

C. Z. - J'aimerais faire du cinéma.

PPDA - Si vous rencontrez Gérard de Villiers au hasard d'un couloir...

C. Z. - La dernière rencontre, il a fui. C'était dans la maison d'édition des Presses de la Cité. Il s'était très mal comporté ; il avait porté des attaques très basses. Le personnage est très bas de toute façon. C'est une des raisons pour lesquelles j'attaque son empire.

PPDA - Et vous avez eu envie physiquement de lui casser la figure?

C. Z. - Trop vieux! Et puis il a fui.

PPDA - Il faut dire que quand on a en face de soi un homme comme vous, on peut avoir peur, si on a bien lu sa biographie.

C. Z. - Non. Là, c'est lui qui a commencé d'une manière déloyale, donc je continue. 
PPDA-Vous avez donc continué en décidant de l'attaquer sur son secteur, c'est-à-dire ce qu'on peut appeler une littérature de gare, sans que ce soit du tout péjoratif, mais c'est beaucoup lu. Là, vous tirez à combien?

C. Z. - Cinquante mille de vendus en cinq semaines.

PPDA - Sans que vous en ayez encore parlé ! Donc, là, après l'émission, ça va encore redoubler ${ }^{22}$.

\section{L'auteur soi-même}

Impliquant une présence sous tutelle du livre à la télévision, l'émission littéraire, on l'a $\mathrm{vu}$, ne se tient que d'instaurer une fonction-auteur inédite, de type juridico-expressif, dont la propriété sui-référentielle peut fonder le régime de signification propre à ce genre d'émission. En d'autres termes, c'est aux fins pratiques de l'émission littéraire que satisfait en priorité l'invention de la catégorie d'auteur : elle assigne d'une part un statut (détaché de celui d'écrivain) à la personne présente sur le plateau, elle lui reconnaît d'autre part des ressources expressives propres et partiellement indépendantes de celles que renferme le livre. La contradiction qui, dès lors, risque de pointer entre le livre et l'auteur se résout pour peu que l'auteur soit également présent dans son livre.

C'est ce dernier point qu'il nous appartient de préciser maintenant, en soulignant tout d'abord qu'il est indissociable des précédents au sens où le dispositif de l'émission littéraire trouve sa forme achevée lorsque la présence de l'auteur sur le plateau redouble sa présence dans le livre. Sous cette condition, l'émission littéraire peut en effet s'invisibiliser comme instance de production-imposition de la catégorie d'auteur, puisque aussi bien le livre en objective alors la réalité préalable. Dès lors, il n'y a pas lieu de s'étonner que les émissions littéraires accordent une audience privilégiée et toujours croissante aux récits de type (auto)biographiques; ce que vient confirmer notre étude sur la part respective des différents genres littéraires à la télévision ${ }^{23}$.

Pour autant, il n'est pas davantage dans notre propos de prétendre inférer la récente vogue éditoriale pour ce genre, des modalités de son traitement médiatique. Car c'est précisément au-delà des limites (encore) étroites du genre (auto)biographique proprement dit que l'on peut prendre véritablement toute la mesure de ce phénomène éminemment médiatique qu'est la généralisation de l'argument biographique, son devenir hégémonique, particulièrement manifeste sur le plateau d'Apostrophes.

B. P. [à J.-M. Rouart, à propos du héros de son roman La Femme de proie] - Est-ce que c'est un masochiste?

J.-M. R. - Je crois que les liens qu'il a eus avec sa mère sont un éclairage sur sa vie amoureuse.

B. P. - Et vous avez vous-même rencontré des «femmes de proie » aussi terribles?

J.-M. R. - Bien sûr, sinon je n'aurais peut-être pas écrit ce livre.

B. P. - Je sais maintenant Jean-Marie Rouart comment vous les séduisez les femmes : dès la première rencontre, vous racontez la vie de Pierre Loti, la deuxième, de Lord Byron, la troisième d'Alfred de Vigny, la quatrième de Romain Gary. Je trouve ça très astucieux de votre part.

J.-M. R. - Mais c'est le héros du roman.

B. P. - Oui, oui, d'accord.

Bernard Pivot interroge ensuite l'auteur de Métisse blanche.

B. P. - Est ce que vous avez gardé de la haine pour ce père que vous n'avez pas connu? 
Enfin, c'est au tour de Daniel Fernandez, auteur du Rapt de Ganymède, véritable plaidoyer pour une culture homosexuelle.

B. P.-Daniel Fernandez, vous avez considéré longtemps que vous ne pouviez pas parler dans vos livres franchement de l'homosexualité, de votre homosexualité. Et c'est vrai que là, vous parlez clair dans ce livre. Mais, est-ce que justement vous n'avez pas pris un titre métaphorique parce que vous n'osez quand même pas encore dans le titre dire carrément les choses ? Vous vous cachez encore un peu. Le Rapt de Ganymède, c'est tellement obscur... ${ }^{24}$.

\section{La confession}

31 Voici donc l'auteur prié de renoncer, comme à une dernière coquetterie, à son ultime effet d'écriture, exilé pourtant déjà hors du texte, et d'énoncer clairement la demiobscurité d'un livre caractérisé pourtant déjà par le « clair parler ».

Pareille mise en demeure vient sceller de manière exemplaire ce qui ne peut manquer d'apparaître en référence à l'intitulé d'un ouvrage polémique de Finkielkraut comme une "défaite de l'écriture ». On retrouve là cette défiance envers le syle de l'écrivain que stigmatise pour sa part J. Starobinski à propos du malentendu qu'ont longtemps entretenu l'autobiographie et la critique littéraire.

Selon la représentation qui voit dans le style une "forme » ajoutée à un «fond » il est en effet loisible de jeter la suspicion sur les qualités de style d'une autobiographie. "Trop beau pour être vrai» devient le principe d'une défiance systématique. A quoi s'ajoute, lié à l'expérience commune de l'usage de la parole, le sentiment du risque permanent d'un glissement dans la fiction. Non seulement l'autobiographe peut mentir, mais la «forme autobiographique» peut revêtir l'invention romanesque la plus libre [...].

Sous l'aspect de l'autobiographie ou de la confession, et malgré le vœu de sincérité, le "contenu" de la narration peut fuir, se perdre dans la fiction, sans que rien n'arrête ce passage d'un plan à l'autre, sans qu'aucun indice non plus ne le révèle à coup sûr. La qualité originale du style, en accentuant l'importance du présent dans l'acte d'écrire, semble favoriser l'arbitraire de la narration plutôt que la fidélité de la réminiscence. Plus encore qu'un obstacle ou un écran, c'est un principe de déformation et de falsification ${ }^{25}$.

Le modèle littéraire mis en œuvre sur le plateau est au contraire tout de transparence. Comme tel il s'inscrit dans le prolongement des confessions de Saint Augustin, véritable coup d'essai autobiographique, dont il constitue en quelque sorte la version laïque, puisque le public des téléspectateurs, est ici substitué à l'allocataire divin qui justifiait le projet autobiographique augustinien. La référence affleure d'ailleurs lorsqu'un autre serviteur de Dieu présente à son tour ses mémoires.

B. P. [au révérend père Bruckberger] - Voilà le dernier tome de vos mémoires... Je dirais plutôt " confessions ", parce que vous y confessez des péchés considérables! D'entrée de jeu, vous confessez le péché suprême, enfin, pour un dominicain, pour un prêtre : pendant dix années, vous avez eu une maîtresse qui s'appelait Barbara. 
Pourquoi commencez-vous ce récit par la confession de Barbara?

B.-Parce que c'était le plus difficile à dire. C'est une chose que je me reproche vivement.

B. P. - Quoi donc?

B. - D'avoir manqué à mon vœu de chasteté. J'en demande pardon à Dieu tout le temps.

B. P. - Mais vous ne regrettez rien! [B. P. lit un passage.] Est-ce que vous n'ajoutez pas au péché de chair le péché d'orgueil ?

B.-Je sais que je suis coupable et je prends la responsabilité de toute ma vie. C'est pour ça que j'ai écrit des mémoires.

B. P. - Mais la grande affaire de votre vie, c'est Barbara ou c'est Jésus-Christ?

B. - C'est Jésus-Christ bien sûr. Sinon je ne me sentirais absolument pas coupable ${ }^{26}$. autobiographique, l'auteur étant sans doute lié au personnage par quelque singulier attachement, à moins qu'il ne le façonne à son image, ce que Bernard Pivot s'emploie à vérifier dans les extraits suivants :

B. P. [(à Edouard Behr, auteur d'une biographie consacrée à Hiro Hito] - Vous, quel est votre sentiment? Vous avez passé des années avec Hiro Hito ; vous l'avez suivi de sa naissance jusqu'à sa mort ; est-ce un personnage... Je ne pense pas que vous l'aimiez, certainement pas, mais est-ce que vous avez de la sympathie pour lui ${ }^{27}$ ?

B. P. [à Élisabeth Roudinesco, psychanalyste, auteur d'une biographie consacrée à Théroigne de Méricourt, figure féminine de la Révolution française] - Est-ce que vous vous seriez passionnée pour Théroigne de Méricourt au point de faire un livre, si elle n'avait pas sombré dans la folie?

É. R. [embarrassée, sous le regard satisfait de B. P.] - Je ne sais $\mathrm{pas}^{28}$.

[...]

B. P. [à la très rousse Régine Deforges, auteur d'une biographie romancée d'Anne de Kiev] - Anne de Kiev est rousse dans le livre; d'ailleurs, toutes vos héroïnes sont rousses.

R. D. - Elles ne le sont pas toutes; mais ce sont les lecteurs qui imaginent qu'elles le sont. Je vous assure que c'est vrai ${ }^{29}$.

\section{La fiction inavouable}

Le principe dramaturgique d'expressivité prend également le même tour réflexif dont on a vu déjà qu'il porte le principe éthique de responsabilité à son accomplissement formel dans le modèle de la confession. Avec l'omniprésence de l'auteur, dans le livre et sur le plateau, le régime de signification institué à partir de la reconnaissance de ressources expressives propres à la personne de l'auteur trouve enfin un critère 
d'appréciation de la qualité tout à la fois du livre et de la performance de son auteur. Critère non pas esthétique mais moral : la sincérité, c'est-à-dire une expression sans arrière-pensée ni faux semblant. Plus exactement, faute de pouvoir apprécier la qualité esthétique du livre ${ }^{30}$, ce sont les qualités morales de l'auteur qui en tiennent lieu; ou du moins celles qui, au terme d'un véritable processus de stigmatisation ${ }^{31}$ sont imputées à l'auteur (catégorie de l'identité sociale) alors même qu'elles se rapportent tout au plus à une identité personnelle. C'est ainsi que face au très courageux Daniel Fernandez qui ose "parler clair ", Jean-Marie Rouart, pris quant à lui en flagrant délit de dénégation du caractère autobiographique de son héros, ne peut manquer d'apparaître comme un faible, qui se camoufle derrière un personnage de fiction. De même, pour n'avoir pas eu le courage de ses opinions, c'est Bertrand Poirot-Delpech qui perd la face, face à la pourtant très obséquieuse Irène (sans) Frein.

Cette sincérité requise de l'auteur porte fondamentalement sur un point : les modalités de sa propre présence dans le livre, étant entendu, par présupposition, que l'auteur comme instance biographique, habite de quelque façon son œuvre. Aussi la présentation du livre se borne-t-elle le plus souvent à une opération de réduction du fictionnel (un réel imaginaire) au biographique (un réel dont la personne bien vivante de l'auteur garantit l'appartenance au monde de la vie quotidienne) qui n'est autre, littéralement, qu'une opération nouvelle de "construction sociale de la réalité » de l'écriture, indépendante cette fois de la mystique de la création attachée à la représentation classique de l'écrivain. L'auteur, pour sa part, ne saurait dans le même temps faire preuve de la sincérité requise et se prétendre auteur romanesque... sauf à apparaître lui-même comme personnage de roman! C'est dans ce paradoxe que se tient l'alternative au devenir autobiographe de l'auteur. C'est dans ce paradoxe en tout cas que se résout, sur le plateau d'Apostrophes une fois encore, un bien singulier dilemme mettant successivement à l'épreuve le principe éthique de responsabilité (par absence de l'auteur susceptible de l'assumer) et la norme expressive de sincérité (par dénégation du caractère biographique d'un soi-disant roman précisément consacré à l'auteur disparu). A l'origine de cette affaire: l'absence sur le plateau de Matthieu Galey, auteur décédé d'un Journal dont le second tome à peine publié par les éditions Grasset défraie la chronique du Tout-Paris littéraire.

B. P. - Geneviève Galey, vous êtes la sœur de Matthieu Galey, vous êtes venue il y a dix-huit mois je crois présenter le premier volume... Alors là, il y a une dimension qui est très poignante, c'est bien sûr la mort de Matthieu Galey, puisqu'il apprend deux ans avant, presque jour pour jour qu'il va mourir, et dans des conditions atroces. D'ailleurs, c'est marqué dans le journal: le 24 janvier 1984, il y a simplement: «Visite au professeur M.». Ce qui est extraordinaire dans ce livre, c'est le courage de Matthieu Galey, sa force de caractère, son humour, sa lucidité ; qui est un peu effrayante d'ailleurs. Dès le début, il dit « attention au cabotinage de l'angoisse ». C'est absolument incroyable! Alors vous, vous saviez?

G. G. - Oui, il me l'a dit; pas tout de suite, l'été 84 [raconte la scène, sans qu'on sache si elle figure dans le livre].

B. P. - Alors, Yves Berger (« responsable» chez Grasset) vous êtes présent bien entendu dans ce livre. Vous savez qu'il est fichu, et vous lui proposez un contrat. Il le raconte.

Y. B. - C'est le lieu ici, je pense, de raconter qu'il a tenu par l'écriture. Il s'est dit : il faut que je fasse ce journal, cette œuvre.

B. P.-Alors Claire Gallois, vous étiez très amie de Matthieu Galey. Il parle de vous aussi dans ce livre. Vous avez su la vérité très vite je suppose.

C. G. - Assez vite. Un jour du mois de mars où j'étais dans le Vaucluse toute seule, il pleuvait. Et il m'a téléphoné pour me le dire. J'ai eu un réflexe très égoïste ; il m'en a 
beaucoup voulu par la suite, c'est que quand il a raccroché, je me suis dit qu'est-ce que je vais faire? J'ai pris dans les pages jaunes de l'annuaire une agence immobilière de Car-pentras et la maison était vendue le lendemain. Alors, il m'a dit : « Tu me lâches ! - Et toi alors »!

B. P. - Mais, à un moment, vous laissez... lui-même n'arrive pas très bien à comprendre quel en est le symbole, une robe sur son lit.

C. G. - Une robe, oui. Il n'a pas compris ! Ben, je m'en allais, c'était fini. Non, il n'a pas compris, j'ai vu dans le journal ; ça valait mieux qu'il comprenne pas.

B. P.-Dans un moment, on parlera du roman de Claire Gallois dont à l'évidence Matthieu Galey est le héros [lit une description].

C. G. - J'ai quand même fait un roman. J'aurais pas eu le culot de dire que j'allais faire un portrait de lui. Je lui avais promis d'une certaine façon: pas longtemps avant sa fin, il me dit est-ce que tu viendras sur ma tombe? « Ah ça non!». Il me dit : « Alors écrit ».

B. P. - Alors, il y a l'aspect médical qui est très émouvant, et puis il y a, comme dans le premier volume, le côté témoignage sur la vie littéraire parisienne et ça, ça fait le régal de pas mal de lecteurs. En tout cas, moi j'en suis... Maurice Rheims, quel est votre avis sur... d'ailleurs je signale que dans le premier volume vous étiez sévèrement égratigné, parce qu'il raconte un déjeuner chez vous, il le qualifie de déjeuner d'ascenseur.

M. R. [de l'Académie française] - Il devait avoir raison... Ce qui m'a touché, il y a peut-être deux ans déjà, c'est la soirée, mais elle ne s'en souvient plus, de Claire Gallois chez François Nourissier, où je l'avais raccompagnée chez elle et où elle s'est mise à me parler de Matthieu Galey. Je ne savais pas qu'il était malade et elle a fondu en larmes.

B. P.-Bien, maintenant passons au document lui-même. La rumeur, et pas seulement la rumeur, mais certains journaux et notamment Le Figaro littéraire de lundi dernier laissent entendre que ce journal n'est pas paru tel qu'il aurait dû paraître et que l'éditeur, notamment vous, Yves Berger, et Jacques Brenner, qui avait été chargé par Matthieu Galey de mettre au point le manuscrit, vous auriez enlevé beaucoup de choses pour ne pas déplaire à certaines personnes de chez Grasset. Alors c'est vrai ou c'est faux?

Y .B. - Écoutez, je suis étonné que des journalistes aient pu agiter ce problème. Il s'agit d'un homme qui meurt, qui se sait condamné, qui découvre qu'il n'a plus la force de corriger son journal, de donner à ce journal l'aspect éditorial qu'il veut lui donner. Ça, il faut quand même se mettre dans l'esprit dans lequel on tient un journal. Je tiens un journal moi, je sais comment ça se passe : on rentre le soir, on écrit vite pour ne pas perdre le sentiment de ce qu'on a vécu, imaginé ou pensé... ou on écrit le matin parce que le soir on est trop fatigué. Et la pensée, après, quand on le relit, les jugements on les trouve un peu trop forts, pas assez justes. Il s'est livré, sur toute une partie de ce qu'il avait écrit et qui correspond au premier tome, à ce travail. Pour le reste, il n'a pas pu le faire ; il voulait que cela fut, il a fait confiance à sa maison d'édition, les éditions Bernard Grasset; je vous rappelle que c'est moi, Yves Berger, qui l'avait fait rentrer chez Grasset, c'était un ami ; et il délègue, il choisit quelqu'un et ce quelqu'un qu'il choisit pour travailler les pages qu'il n'a pas pu travailler et dans l'esprit où lui les aurait faites, c'est Jacques Brenner qui est une personne, un écrivain, dont la probité, le sérieux, sont incontestables et ne sont niés par personne.

B. P. - Autrement dit, contrairement à la rumeur parisienne qui dit que vous avez coupé des lignes parce qu'elles n'étaient pas agréables sur...

Y. B. - Mon cher Bernard Pivot! Personnellement, je n'ai pas fait de coupes, c'est Jacques Brenner qui a fait ce travail.

G. G. - Franchement Yves, faut pas dire ça [révèle l'existence de coupes imposées par l'éditeur].

B. P. - Mais c'était quoi, ces coupes?

G. G. - Ben, faut être clair : un certain nombre de magouilles sur l'attribution de 
certains prix, notamment le Goncourt ; c'est vrai, des Goncourt Grasset ! Et aussi des jugements littéraires sur certains auteurs Grasset! C'est ça où je n'étais pas d'accord.

B. P. - Mais est-ce que vous pensez que ce journal est défiguré ?

G. G. - Non, pas du tout, mais je regrette qu'on ait coupé comme par hasard les jugements sur des auteurs Grasset.

.P. - Cela dit, ça représente combien?

G. G. - ...une dizaine de pages.

Y. B. - Une dizaine sur deux mille, vraiment !

B. P. - Venons en au livre de Claire Gallois. Toujours dans le même article du Figaro littéraire que vous avez signé, vous traitez le roman de roman de nécrophage. Qu'est-ce qui vous gêne dans le roman?

G. G. - De deux choses l'une, ou c'est un roman et alors on dit que c'est Matthieu Galey...

Y. B. - Mais ce n'est pas elle qui l'a dit.

G. G. - Mais, il y a des papiers tous les jours.

C. G. - Geneviève, soyez franche, tous les journalistes que j'ai rencontrés, vous étiez passée avant.

Y. B.-Vous avez même demandé des coupes dans son roman!

M. R. - Je tiens à dire que j'ai lu ce livre par deux fois, qu'il est un véritable roman, que le portrait qu'elle fait de Matthieu Galey est fait avec infiniment de sensibilité, infiniment de talent; et que si j'étais très proche de Matthieu

Galey, je ne serais gêné en rien par le portrait qu'elle a fait qui est un très beau portrait littéraire.

G. G. - Le portrait de Matthieu ne me gêne pas du tout. La seule chose c'est que dans l'entourage de Matthieu, il y avait des gens admirables. La mort de Matthieu, c'était dur à supporter. Ils sont assassinés.

C. G. - Mais, vous ne savez pas lire un roman!

B. P. - Alors, vous l'avez expliqué tout à l'heure, Matthieu Galey vous a demandé d'écrire ce livre.

C. G. - Oui, je ne voulais pas aller sur sa tombe.

B. P. - Mais si je comprends bien, vous y êtes allée tout de même parce que d'après le livre, vous êtes sur le corbillard qui emmène... enfin l'héroïne.

C. G. - Oui, enfin ça c'est peut-être ce qui gêne le plus Geneviève, parce que ça c'est vrai. J'étais toute seule et elle qui est si convenable n'y était pas! En fait, je ne voulais pas mettre cette fin et c'est François Nourissier qui m'a dit : « Ton livre n'est pas vraiment fini. » Mais ça allait tellement bien avec ce qu'on avait vécu ensemble : la seule fois où nous passons une nuit ensemble, il faut qu'il soit dans son cercueil !

B. P. - Alors il vous dit sans arrêt: tu ne me connais pas, tu ne me connaîtras jamais.

C. G. - Il me le disait en vrai. Moi, qu'est-ce que j'ai inventé ? Moi, je voulais raconter l'histoire que je trouve très étrange d'un homme et d'une femme qui ne sont pas faits pour se rencontrer et qui ne pourront jamais se rencontrer.

B. P. - Oui, parce que de toute façon l'un est homosexuel, c'est Matthieu Galey, et que...

C. G. - Mais c'est pas forcément Matthieu !

B. P.-Mais bien sûr que c'est lui ! Bon, mais attendez... c'est vrai que le roman est intéressant, parce qu'on est sur l'ambiguïté des rapports d'une femme qui est très amoureuse d'un homme, et d'un homme qui de toute façon ne peut pas apporter grand chose à cette femme parce qu'il aime des hommes.

C. G. - Ça c'est un vrai sujet de roman!

B. P.-C'est vrai, moi je trouve que votre roman est un beau roman. Simplement, ce que j'aime moins, c'est cette sorte d'hypocrisie à dire c'est un roman alors qu'il n'y a rien de romanesque.

C. G. - C'est pas vrai, j'ai inventé énormément de scènes.

B. P. [sous le regard complice de G. G.] - Ouais, je veux bien, mais même dans le 
fourgon mortuaire, même là vous y êtes !

C. G. - Ben là oui, bien sûr !

G. G. - C'est nous qui aurions dû écrire un roman là-dessus ! C. G. - C'est vous qui auriez dû être dans le fourgon! G. G. - Oui, c'est vrai.

M. R. - La vérité, c'est que Matthieu aime Claire comme un personnage de roman ; c'est ça qui apparaît dans son journal. Elle l'amuse, elle l'étonne avec sa gouaille.

Elle est un personnage du roman qu'il n'a pas écrit.

[G. G. approuve ${ }^{32}$.]

La chose est suffisamment remarquable pour être soulignée : par deux fois, la fiction est proprement constitutive de l'auteur en tant qu'unité biographique. C'est tout d'abord par un roman que l'auteur disparu du Journal se réalise biographiquement; c'est son existence comme héros d'une intrigue typiquement romanesque ("ça c'est un vrai sujet de roman») qui vient finalement suppléer à son absence physique en tant qu'auteur assumant naturellement la responsabilité de son livre. C'est ensuite parce qu'elle est reconnue comme personnage de roman que Claire Gallois peut se dire en toute sincérité romancière alors même que le caractère biographique de son livre est démontré.

\section{L'auteur tel qu'en lui-même. L'idéal de l'authenticité}

Susceptible de faire bon ménage avec la fiction, ce principe moral de régulation expressive qu'est la sincérité est au service d'un idéal, non pas de vérité mais d'authenticité. $\mathrm{Au}$ lieu d'engager, dans leur bien-fondé, les propositions dont la personne invitée sur le plateau est l'auteur, et de devoir pour ce faire présupposer leur vérificabilité, l'authenticité recherchée ici concerne la personnalité même de l'auteur telle qu'il la donne à voir en public. Elle se mesure tout simplement à la plus ou moins grande conformité entre ce que dit (ou laisse entendre) l'auteur sur le plateau et ce qu'il a écrit noir sur blanc (ou entre les lignes) dans son livre. Ou en d'autres termes, entre un livre devenu document sur la personne de l'auteur, et un auteur sur le plateau incarnant l'effigie de sa propre personne. Ainsi, l'authenticité, à son tour, n'est jamais aussi grande que lorsque l'auteur sur le plateau incarne un personnage de son roman. A cet égard, la prestation réalisée, lors d'un numéro d'Apostrophes consacré aux étrangers, par Azouz Begag jeune auteur fils d'immigré algérien, demeure un modèle du genre ${ }^{33}$.

B. P. - Azouz Begag, vous, vous ne venez pas d'un pays étranger puisque vous êtes né à Villeurbanne ; mais vous êtes toujours algérien...

A. B. [sans cesser d'arborer un sourire complice, comme sur le point d'éclater de rire] - Je suis français depuis juillet-août. [Il raconte l'histoire de sa naturalisation ponctuée d'un « aussi ai-je décidé de prendre la nationalité française. ».]

B. P. - Alors votre roman! Le héros est un lycéen, il s'appelle Ben Abdallah; il a d'ailleurs un problème, il est un peu gros et puis il préfère qu'on l'appelle Béni, comme ça il se fait un peu oublier.

A. B. - Right ! Non, en fait son problème c'est que quand on a seize ans et qu'on est gros, ça craint un peu. Moi, ce que je voulais dire à travers l'histoire de Ben Abdallah, le " gris » hein, c'est que même si on désire fortement s'intégrer dans la société française il y a toujours une porte de ce paradis qui reste fermée. Mais je voulais pas pleurer.

B. P. - Oui, votre roman, il est à la fois émouvant et drôle, parce qu'à un moment donné, Béni devient amoureux d'une fille blonde qui s'appelle France (sic!), si bien qu'il devient marginal dans sa propre famille parce que, bien entendu, son père prend ça très mal qu'il se fasse défriser [on voit A. B. se passer la main dans les cheveux qu'il a frisés, en souriant]. Il devient à la fois marginal dans la société mais 
aussi chez lui.

A. B. - Oui, c'est ce que je suis encore [avec le même sourire].

B. P. - Ce que vous êtes encore?

A. B.-Oui, parce que dès l'instant où j'ai commencé à fréquenter l'université j'ai commencé à manier le concept, à manier l'idée [mimique pour souligner l'autodérision], on est forcé de se détacher de sa communauté d'appartenance.

B. P. - Oui, mais à un moment il dit entre France, la jolie blonde, et mon père, j'ai choisi.

A. B. - [avec enthousiasme] Le père, j'espère !

B. P. - Non, la blonde.

A. B. - [épaté] Ah, il a choisi la blonde?

B. P. - Vous savez bien qu'il a choisi la blonde.

A. B. - Oui, mais y a mon père qui m'écoute, je suis obligé. [Rit tout en regardant de côté vers le public]

B. P. - Oui, mais votre père, il a lu le livre.

A. B. - Il sait pas lire, il est analphabète.

B. P. - Il y a des scènes, je dis pas que c'est votre père, mais le père veut le marier à une Algérienne et là c'est l'engueulade [lit la scène].

A. B. - Là, c'est très difficile [soudain sérieux]. C'est vrai que pérenniser sa présence sur terre, pour mon père, ça signifie pérenniser son sang, sa culture ; et dès l'instant où sa progéniture est en train de s'engager vers la France il commence à avoir peur et donc il commence à me retenir et donc il commence à devenir très sévère envers... envers...

B. P. - Mais en même temps quand il a vu que son fils a fait de bonnes études, que son fils a réussi, il doit être très fier quand même.

A. B. - Très fier, mais il le montre pas. On sait jamais, le mauvais œil ! J'ai dit papa, ce soir je vais passer avec Bernard Pivot à Apostrophes, il dit : «Dis-le à personne » [rires].

Julia Kristeva [universitaire, invitée pour son essai Étrangers à nous-mêmes] - J'ai été très sensible à cette position entre deux chaises qu'occupe le narrateur ; parce qu'il est à la fois attendri par ses parents et leurs fautes de français et à la fois attiré par le nouveau pays; et à travers cet attendrissement, il y a un humour qui est l'autre face du désarroi.

B. P. - D'ailleurs Béni reprend ses camarades quand ils font des fautes de français.

J. K. - Ah oui, il connait le français mieux que les autres. [A A. B.] « Aussi voudrais-je vous dire cher Monsieur » : il n'y a que vous qui savez dire ça!

Tzvetan Todorov [universitaire, auteur de Nous et les autres] - Je trouve que c'est très bien dans ce livre qu'on ne sombre pas dans le sentimentalisme.

A. B. - Ben, je dirais du bien de vos livres aussi [rires].

B. P. - Bravo, quinze-zéro! [Pendant que A. B. se retourne vers le public, comme pour dire à un proche : j'ai drôlement assuré !]

Enfin, comme Bernard Pivot fait parfois la grâce à certains de ses invités de leur offrir un des ouvrages qu'il passe brièvement en revue en fin d'émission, le moment venu Azouz Begag le sollicite à deux reprises, commentant ainsi son sans-gêne : "Je prends le plus cher!" " J'ai pas honte, hein!"

Encouragé, il faut le souligner, par l'ambiguïté présidant à l'intitulé même de l'émission ( «L'étranger », est-ce le thème des ouvrages réunis sur le plateau, ou le statut de leur auteur?), Azouz Begag transforme la présentation de son livre en un double témoignage. Tout d'abord en se rendant proprement équivalent à son héros par une manipulation du dispositif énonciatif (i.e. une transformation constante du «il» des questions de B. P. en «je ») que pointe d'ailleurs implicitement J. Kristeva (lorsqu'elle entend considérer le narrateur indépendamment de l'auteur) avant de s'en faire ellemême la complice (« Aussi voudrais-je vous dire cher Monsieur », il n'y a que vous qui 
savez dire ça). Ensuite en affichant dans la manière de se tenir, dans la façon de parler, cette impertinence par laquelle les jeunes issus de l'immigration maghrébine marquent d'ordinaire leur entrée sur la scène publique. Authentiquement étranger, (pur «beur » en l'occurrence), jusque dans sa personne même, l'auteur témoigne bel et bien d'une personnalité authentique ne sacrifiant rien de sa spontanéité au respect des règles implicites de l'émission. En fait, c'est cette authenticité, la sienne propre, qui lui vaut d'être félicité pour son livre... et de disqualifier implicitement en retour ce type de jugement porté sur le livre mais qui concerne en réalité la performance de son auteur : «Puisque vous avez dit du bien de mes livres, je dirais du bien de vos livres aussi »!

Dès lors que sur le plateau l'authenticité est promue de la sorte comme qualité littéraire suffisante, la distinction que nous avions opérée et qui pouvait alors sembler relever d'un simple jeu sur les mots, entre écrivain et auteur, est réalisée objectivement sur le mode de la séparation définitive. Peu importe, en fin de compte, que l'auteur ait effectivement écrit son livre, ou simplement délégué ce qui n'est plus qu'une compétence technique à quelque "nègre ». Depuis le journaliste sportif mettant sa plume au service de tel champion dont on peut aisément concevoir qu'il ne puisse " écrire sa légende " tout seul, jusqu'à l'entrepreneur ès lettres rentabilisant à coups de best-sellers son officine littéraire, nombreuses sont en effet les formules de « collaboration ». Loin de remettre en question la légitimité de l'auteur (lequel continue d'assumer pleinement ses fonctions juridico-expressives) elles l'assignent d'autant plus à sa propre réalisation biographique qu'elles le libèrent de la contrainte d'écriture. Simplement faut-il pour ce faire que l'auteur paraisse publiquement, c'est-à-dire que le signataire vienne gagner sur la scène publique ses galons d'auteur. Scellant ainsi de manière définitive ce que les éditeurs "négriers" ont coutume d'appeler la «réappropriation de l'ouvrage» par son auteur ${ }^{34}$. Dans ces conditions, la scène médiatique ne saurait être conçue comme un espace de simple représentation, mais plutôt comme un espace d'accomplissement. Ce qu'a parfaitement assimilé P.-L. Sulitzer, comme en témoigne le film publicitaire consacré à son roman: Les Routes de Pékin $^{35}$. On y voit le personnage P.-L. Sulitzer incarné par P.-L. Sulitzer lui-même, confronté à cette même question : "Etes-vous bien l'auteur de vos livres ? ", à laquelle une enquête réalisée par le magazine Lire (dont le directeur n'est autre que Bernard Pivot) avait donné une réponse définitive. Et qui n'est autre ici qu'une de ces questions stéréotypées que suscitent les vedettes et dont la presse à sensation se fait l'écho. Il s'agit là de la reformulation particulièrement mesquine et ne méritant en conséquence pas de réponse, de cette question autrement plus déterminante : "Qui est l'auteur? ». Question qui, pour n'être pas formulée, n'en reçoit pas moins en revanche une réponse sans ambiguïté à l'écran: l'auteur n'est nul autre que celui qui paraît sur la scène publique pour y répondre de son livre!

\section{Pour être écrivain, on n'en est pas moins homme}

Spécialement conçue, dans son dispositif (qui tend, rappelons-le, à faire de l'auteur l'incarnation métonymique du livre), pour ne pas avoir à y figurer la question de l'écriture, l'émission littéraire de plateau est devenue le haut-lieu de la réalisation biographique de l'auteur. Nul besoin d'être écrivain et mieux encore, nul besoin d'avoir soi-même écrit son livre pour y paraître. D'ailleurs l'écrivain véritable refuse de s'y commettre, ou alors est immanquablement saisi par la gêne s'il s'y aventure. En 
d'autres termes, si l'émission littéraire participe du processus de désignation sociale de l'écrivain, "sacré écrivain", pour reprendre l'expression de Bernard Pivot, ce serait par défaut. En ces temps d'indétermination quant au statut des personnes invitées, l'absence des plateaux, ou du moins la gêne à s'y produire, pourrait bien être la dernière marque distinctive de l'écrivain! Il n'est pas étonnant, dans ces conditions, que, tout occupés à saturer l'écran de leur présence corporelle, de l'expression de leur visage, de l'exposé de leurs idées ou du récit de leur histoire (la leur propre ou celle de leur héros), les auteurs invités n'y parlent pratiquement jamais du travail, du plaisir ou encore de la discipline de l'écriture. A moins que l'écriture elle même ne constitue précisément un événement de leur propre biographie, sur le mode par exemple de cette véritable conversion dont témoigne la comédienne Annie Girardot sur le plateau d'Exlibris.

PPDA - Nous allons tout de suite recevoir Annie Girardot. Annie Girardot, parce qu'elle vient d'écrire un livre chez Robert Lafont; un livre qui lui ressemble : Vivre d'aimer. C'est-à-dire que ce n'est pas un livre de star, c'est vraiment un livre de femme. C'est un compliment qu'on peut vous faire.

A. G. - Ça fait cinq ans que je voulais le faire et je n'y arrivais pas. Pour moi, écrire c'était d'un seul coup se mettre à sa table devant une feuille blanche et là je suis incapable de dire quoi que ce soit. Et puis, brusquement j'ai découvert, il y a un an à peu près, et d'un seul coup je me suis mise à écrire. Je me suis dit « Mais qu'est-ce qui m'arrive?». Alors, je l'ai toujours écrit dans quelque chose qui est en mouvement: dans un avion, dans une voiture, dans ma voiture, dans le train; ou bien toujours en marchant, en courant, avec ce que j'avais sur moi. Alors c'était des mots croisés, et j'écrivais dessus comme ça, je sais pas pourquoi. Je me suis aperçue que j'étais libre d'écrire parce que dans le mouvement ça veut dire que j'étais pas chez moi avec tous les tracas. Là, je suis disponible et l'écriture devient... enfin, pas l'« écriture », parce que moi, j'écris comme ça ; mais brusquement, j'écris comme un médium. C'est assez surprenant d'ailleurs ce qui m'arrive ${ }^{36}$.

Une découverte: voilà ce qu'est l'écriture pour cet auteur qui se surprend soi-même écrivant (lui-même); voici plus largement ce que ne peut manquer d'être la moindre parution télévisuelle d'un écrivain digne de ce nom. On en trouvera la confirmation exemplaire dans cette alternance, caractéristique d'Apostrophes, entre des émissions standard (émissions de plateau, consacrées à plusieurs livres à la fois) et une émission spéciale, périodiquement consacrée à un seul écrivain, qui fait à Bernard Pivot l'honneur de son domicile, pour un long entretien en «tête-à-tête ». L'existence même d'une telle formule suffit en effet à reconnaître implicitement que l'écrivain digne de ce nom n'est pas le personnage public qu'est au contraire l'auteur, et qu'il ne saurait en conséquence ne sortir qu'à titre exceptionnel et dans un cadre assurant le respect de son intégrité, de la retraite supposée être la sienne. C'est d'ailleurs ce même isolement de l'écrivain que soulignent à chaque fois les premières images de ces Apostrophes horssérie. Comme celles de la rencontre Soljenitsyne-Pivot en pleine forêt, non loin de la maison du prix Nobel qui ne peut être filmée " pour des motifs évidents de sécurité ", et dont on sait seulement qu'elle se trouve à proximité d'un village perdu de la Nouvelle-Angleterre qui a pour nom Cavendish ${ }^{37}$. On se souvient aussi de Marguerite Yourcenar, enveloppée de son châle, dans la lande battue par les vents de la petite île des monts Déserts qui l'abrite depuis bien des années, avec ce commentaire en voix off de Bernard Pivot: "Loin de Paris, elle s'est hissée au premier rang des auteurs de langue française à la seule force de son poignet d'écrivain ${ }^{38}$. " Une telle fidélité à la figure archétypale de l'écrivain, auquel on concède d'emblée le privilège d'un cadre et d'un dispositif appropriés, laisse augurer d'un traitement spécifique. Or, et c'est en cela 
que les émissions en «tête-à-tête » d'Apostrophes sont exemplaires : si d'un côté, elles se démarquent des émissions standard, en accordant une place à part à l'écrivain digne de ce nom, c'est qu'à cette condition elles peuvent au contraire, de l'autre côté, l'exposer à son tour à la même entreprise de réalisation biographique, par où l'existence et la personne de l'écrivain deviennent le motif même de sa production littéraire. Et ce en une sorte d'arraisonnement biographique de l'écriture, dont ces deux émissions citées pour leurs prémisses constituent deux modalités symétriques.

\section{Place à l'écrivain}

Dans le premier cas de figure, l'écriture est d'abord prise en compte dans sa matérialité, pour mieux venir ensuite prendre place dans la biographie de l'écrivain à découvrir. Car cette émission consacrée à Soljenitsyne doit en premier lieu être l'occasion de découvrir enfin l'écrivain qu'a trop longtemps occulté, de l'aveu même de l'intéressé, la célébrité acquise par le rescapé du goulag.
A. S. - J'ai un destin malheureux à cause de mes déclarations politiques. A cause de ces déclarations publiques, presque tous les critiques me prennent pour un homme politique. Je n'ai lu que des jugements sur mes prises de position politiques, je ne connais pratiquement pas d'articles critiques sur mon œuvre [...]. Plus j'avance en âge, plus je suis triste d'être célèbre non pas pour mon activité principale mais pour mon activité secondaire.
B. P. - Puisque vous êtes un écrivain, parlons de votre travail d'écrivain : on voit bien que vous êtes un passionné de vocabulaire.
A. S.J'ai travaillé la langue russe pendant 35 ans, j'ai passé tout mon temps à ce travail aux camps.
[La caméra montre de vieux carnets littéralement noircis d'une écriture minuscule, à peine déchiffrable; puis des manuscrits d'aujourd'hui recouverts de la même écriture.]
B. P. - Pourquoi écrivez-vous aussi petit, maintenant que vous êtes libre, que quand vous étiez aux camps?

Voici donc finalement qu'il est question d'écriture, et doublement: comme archive, puis comme trace d'une monoactivité dont l'ancienneté n'a d'égale que la constance ; comme manière d'écrire ensuite, comme stigmate d'une vie carcérale. C'est en son sens religieux qu'il faut prendre ici la métaphore; Bernard Pivot terminant d'ailleurs l'entretien par cette question: "Est ce que vous avez l'impression, peut-être même physique, d'écrire sous le regard de Dieu?»

Dans ces conditions, parler enfin d'écriture, ce n'est jamais que parler encore et toujours d'une vie qui nous apparaît, par là même, vouée à l'écriture. En d'autres termes, le travail de l'écriture se confond ici avec le travail de remise en ordre biographique par où l'écriture vient intégrer une biographie dont elle constitue en retour, la totalisation synthétique. Du reste, si l'épreuve de la liberté par l'exil a transformé la vie de Soljenitsyne, alors même qu'entre "l'archipel du goulag » et la terre d'asile, la réclusion est simplement devenue volontaire, c'est parce qu'elle a transformé les conditions dans lesquelles il peut aujourd'hui accomplir son travail d'écriture.

A. S. - Je trouve vraiment étonnant que l'opinion publique et en particulier des écrivains trouvent anormal qu'un écrivain s'enferme pour écrire. Pour eux, c'est de la folie. On considère qu'un écrivain doit faire des déclarations, qu'il doit paraitre partout. A soixante-cinq ans, pour la première fois, j'ai deux ou trois tables pour écrire et mettre des papiers sans craindre le KGB. Je travaille tranquillement, on 
considère que je suis devenu fou. En fait, on me reproche une vie nouvelle d'écrivain.

Dans le second cas de figure, l'écriture est entendue cette fois comme style. «Je dirais que c'est une écriture janséniste », lance enthousiaste Bernard Pivot (suite à sa lecture d'un court extrait ponctué d'un: "C'est superbe!») pour qualifier le style de Marguerite Yourcenar. Ce style classique dénué d'ornementation qui n'appartient qu'à elle, tout à la fois anachronique et sans âge, comme s'il n'était pas de son (de notre) époque, pas plus que ne le sont d'ailleurs les divers Hadrien, Zénon ou Alexis, héros d'une œuvre pourtant intégralement autodiégétique (i.e. écrite à la première personne). Comme si derrière l'écriture il n'y avait aucune personne qui fut notre contemporain. "Ça c'est curieux, remarque Bernard Pivot avec cette candeur feinte qui le caractérise, que vous puissiez dire que vous connaissez mieux un empereur du $\mathrm{II}^{\mathrm{e}}$ siècle après J.-C. que votre père. » En face d'un tel dispositif littéraire d'effacement de la personne de l'écrivain (à ce point consubstantiel de l'écriture que le tout premier livre, paru en 1929, était déjà signé "Yourcenar », pseudonyme obtenu par anagramme du véritable patronyme de Marguerite : Crayencour), l'émission consacrée à Marguerite Yourcenar doit permettre, à l'inverse de la précédente, de découvrir enfin la femme derrière l'écrivain consacré (elle sera reçue l'année suivante à l'Académie française, en faisant du reste la une de l'actualité parce qu'il s'agissait d'une femme !) ; l'émission doit faire encore découvrir cette femme dont on peut dire qu'elle a consacré sa vie à l'écriture. Bernard Pivot termine d'ailleurs l'entretien par cette question : « Est-ce que laisser des livres sur cette terre ce n'est pas aussi beau que de laisser des enfants?» Ainsi est-ce par le biais de l'écriture que s'accomplit ici la réalisation biographique de l'écrivain. Très exactement, par l'intermédiaire de la lecture par Bernard Pivot de phrases écrites, c'est-à-dire proprement par la citation, laquelle permet tout à la fois de marquer la déférence vis-à-vis du style de l'écriture et de restituer à leurs auteurs les propos auxquels Marguerite Yourcenar se voit confrontée. En sorte que, fragment après fragment, c'est l'œuvre entière qui vient donner sa consistance biographique à la personne de l'écrivain; le parcours dans l'œuvre n'étant autre que le détour nécessaire pour y parvenir. Voici quelques éléments de ce "grand jeu de l'oie » de la réalisation biographique littéralement constitué comme tel par la lecture de l'œuvre opérée par Bernard Pivot.

B. P. - Est ce que vous diriez, comme Hadrien : « Ma vie m'émouvait moins qu'une histoire?»

[...]

B. P. - Hadrien dit : «C'est en grec que j'aurais pensé et vécu. » Est ce que ce n'est pas un petit peu vrai pour vous aussi?

[...]

B. P. - Dans Feux qui est l'ouvrage où vous vous confiez le plus, vous dites : «Qu'il eût été fade d'être heureux. » J'ai rapproché ça d'une phrase d'Alexis qui avait été écrite une dizaine d'années avant: "Il est difficile de ne pas se croire supérieur quand on souffre davantage... la vue des gens heureux donne la nausée du bonheur. »

[...]

B. P. -Et cette alimentation [i.e. celle de Zénon qui ne mange pas de viande], c'est aussi la vôtre?

M. Y. - C'est la mienne.

B. P - Je m'en doutais un peu. Et pourtant, je crois que c'est dans Archives du nord, vous dites qu'il vous arrive de manger du jambon.

M. Y. - Non, c'est Hadrien [qui le dit].

[B. P. lit la page 167 des Archives du nord qui lui donne raison !] 
Enfin, il nous faut ménager une place à part à l'émission consacrée à Marguerite Duras $^{39}$, à l'occasion de la parution et du succès foudroyant de L'Amant, son premier livre délibérément autobiographique ${ }^{40}$. Voilà, en effet, un écrivain que l'on croyait connaître et qu'il nous faut redécouvrir dès lors que dans ce livre, Marguerite Duras prend l'initiative de se révéler biographiquement. Ou, dans les termes mêmes de l'entrée en matière de Bernard Pivot : « Voilà que cet auteur singulier, un peu singulier, inclassable - certains, par commodité, l'ont rangé sous la bannière un peu floue de la modernité -, voilà que cet auteur obtient un succès populaire inouï avec son dernier livre: L'Amant. Eh bien, c'est la preuve que Marguerite Duras sait encore nous surprendre. Et sa présence ici ce soir à Apostrophes, c'est aussi une bonne surprise. » En dépit de cette prémisse, Bernard Pivot se laisse d'abord prendre au piège de la continuité de l'œuvre, et reprendre par une Marguerite Duras qu'il croyait à son tour pourtant déjà connaître.

B. P. - Vous, vous êtes une « fouilleuse d'amours »; vous n'arrêtez pas! Vous n'avez pas cessé de fouiller!

M. D. - Vous confondez avec une autre là !

B. P. - Comment ça ? Non, vous!

M. D. - J'ai rien fouillé. Tout est imaginaire. C'est que des fictions mes livres. C'est la première fois que je...

B. P. - Oui, mais tout se retrouve. Cette histoire (Le. celle de L'Amant) se retrouve dans tous vos livres. On retrouve les personnages des livres précédents: le viceconsul, Anne-Marie Stretter...

51 A cela, Marguerite Duras, elle-même, mais dans un autre lieu, pourrait fort bien répondre: "Les romans antérieurs avaient dessiné en creux le lieu du texte autobiographique; celui-ci les cite donc, les revisite. La mémoire renoue l'avant et l'après, indique les points où était ancrée la fiction - et voici de nouveau mais autres la mendiante, le vice-consul, Anne-Marie Stretter ${ }^{41}$. » On veut marquer, par cet emprunt à la critique littéraire qu'ici, la visée implicite d'«arraisonnement biographique » de l'écriture, doit compter avec la spécificité d'une écriture précisément déjà autobiographique. Aussi, la lecture biographique de l'œuvre antérieurement pratiquée auprès de Marguerite Yourcenar, avec l'efficacité que l'on sait, et tentée à nouveau par Bernard Pivot, vient-elle buter sur les dénégations fermes de Marguerite Duras. C'est finalement à partir de la prise en compte de la rupture provoquée au contraire par l'événement de l'autobiographie que l'émission peut une nouvelle fois réaliser cette réduction biographique de l'écriture qui est l'envers de l'avènement biographique de l'écrivain.

B. P. - L'Amant commence par deux pages sur votre visage. Alors c'est intéressant parce que là les téléspectateurs voient votre visage.

M. D. [en riant] - Je vais regarder la caméra.

B. P. [lit un extrait : «J'ai le visage détruit »] - Vous croyez que vous avez un visage détruit?

M. D. - J'ai la peau du visage détruite, oui.

[...]

B. P.-L'Amant est le signe tangible de votre retour à la vie [i.e. après une cure de désintoxication] ; c'est une résurrection. Il y a une jubilation de votre écriture dans L'Amant, et je me suis demandé si ça n'était pas que vous aviez échappé à tout ça, que vous reveniez de l'enfer; parce que vous revenez de l'enfer!

Ce n'est qu'en faisant littéralement «mauvaise figure » à la télévision que l'écrivain acquiert ici consistance biographique ! En ceci l'émission consacrée à Marguerite Duras constitue un vertigineux raccourci de l'efficacité de l'écran sur l'écrit, puisque la seule 
parution du visage de l'écrivain à l'écran suffit à « l'arraisonnement biographique » de l'écriture : c'est simplement parce qu'on voit cette figure détruite que l'écriture peut être exclusivement qualifiée de «jubilatoire », et le livre de «signe tangible de retour à la vie ".

Notre investigation touche à son terme avec ce retournement, le dernier d'une longue série, qui porte paradoxalement à son comble un effet que l'on aurait pu imputer aux seules émissions de plateau. Ce qui était supposé être un espace protégé pour la parution télévisée d'écrivains dignes de ce nom, s'avère plutôt le théâtre d'un véritable " arraisonnement biographique » de l'écriture. Par l'intermédiaire duquel se réalise une incarnation biographique de l'écrivain d'autant plus "personnelle » qu'elle est l'œuvre d'une lecture et non de la simple efficacité du dispositif assurant la présence du livre sur le plateau. D'autant plus authentique aussi qu'elle paraît s'opérer à l'insu même de l'écrivain, alors que l'auteur sur le plateau se définit à l'inverse par son engagement sans retenue dans cette opération ${ }^{42}$.

\section{La figuration d'une absence}

Il nous reste cependant à considérer, à titre de contre-exemple, certaines productions (les collections Préfaces, Les Hommes-livre $s$, la série Océaniques), s'inscrivant délibérément en marge des émissions littéraires ordinaires.

D'abord parce qu'elles peuvent satisfaire à d'autres finalités que la seule diffusion télévisée : archivage bien sûr, mais aussi constitution d'un fond de vidéocassettes. En effet, « depuis plusieurs années, la Direction du livre et de la lecture (DLL) du ministère de la Culture s'efforce de prouver par son action en faveur des vidéothèques, au sein des bibliothèques publiques, que l'on peut développer ensemble, harmonieusement, le goût de la lecture et l'amour du cinéma ${ }^{43}$ ». De sorte que ces productions sont à proprement parler des coproductions associant le plus souvent une chaîne de télévision et l'INA, avec la participation de la DLL.

Ensuite parce qu'elles ne se conforment pas à chaque fois à un dispositif standard qui est celui de la série télévisée. Tout au contraire : «Les portraits d'écrivains doivent sur un mode non écrit, par la manière de filmer et de dialoguer, par la mise en scène de l'entretien même, chercher à faire écho à l'œuvre elle-même. Tenter de l'incarner à travers la présence et la parole de l'auteur pour placer déjà le spectateur sur le terrain du livre, ou du moins dans ses parages [...]. Avec les moyens propres au cinéma, on peut donner envie de lire ${ }^{44}$.»

En d'autres termes, il s'agit de promouvoir une véritable écriture cinématographique, seule susceptible de faire écho à l'écriture des œuvres... et de dépasser ainsi les limites inhérentes aux simples émissions télévisées. Car l'analogie qu'il convient de réaliser ici, entre l'image de l'écrivain figurée par le réalisateur et son œuvre, se fonde précisément sur la reconnaissance du caractère illusoire de celle-là qui s'opère sur le plateau des émissions littéraires par la simple efficacité de leur dispositif, entre la personne même de l'écrivain et le livre. Si là, tout se joue en direct, ici « tout se joue au montage » ainsi que l'affirme J. Prieur, producteur à l'I-NA, à propos de la collection Les Hommes-livres, comme pour mieux établir encore le rôle déterminant du réalisateur qui n'est autre ici que le promoteur et l'auteur du film consacré à un écrivain dont il est précisément luimême un lecteur passionné. C'est cela d'ailleurs qui lui permet de ne pas être tributaire du système de questions de l'interviewer (familier de l'œuvre, tout comme lui) et de 
déjouer le système de défense que l'écrivain lui-même ne peut manquer d'opposer à qui vient le tirer de sa retraite. «Il faut, dit encore J. Prieur, que le film se fasse malgré l'interviewé et contre l'interviewer ${ }^{45}$.»

58 Encore à découvrir : voici ce que sont les écrivains dont il est question ici. Non cependant que la faute en incombe aux seules émissions littéraires de plateau, l'incapacité à leur ménager une place étant le fait des émissions de télévision en général. Sur ce point également, le projet de la collection Les Hommes-livres est un modèle du genre. «Proposée par L'INA, cette collection de films veut faire connaître les grands écrivains français ou de langue française, auteurs vivants parvenus à la plénitude de leur œuvre mais qui, jusqu’à présent ont été méconnus par la télévision, qu'elle ne leur ait pas accordé la place qui leur revient, qu'elle les ait ignorés ou qu'euxmêmes s'en soient délibérément écartés comme souvent. " Mais alors, à la ligne de clivage qui s'instaure entre émissions (de télévision) et films (de cinéma), vient correspondre une distinction entre les écrivains eux-mêmes. Et qui loin d'en être simplement l'effet, comme une première lecture tendrait à le faire croire, doit être considérée au contraire comme l'opposition originelle en ce qu'elle touche à l'ontologie de l'Ecriture. La distinction dernière entre écrivains connus et inconnus ne saurait dissimuler la véritable opposition, celle du moins qui est posée comme telle, entre les écrivains qui acceptent de se réaliser biographiquement ${ }^{46}$ et les autres qui s'y refusent en derniers fidèles d'une religion de l'écriture qui ne leur survivra pas. Effectivement, «bientôt, on ne verra plus d'écrivain invisible comme Blanchot, décidé à ne jamais apparaître, à ne pas se réaliser biographiquement, à rester consubstantiel au livre. Bientôt disparaîtront Cioran, Beckett et Gracq, habitués à la discrétion, vivant en écrivains déjà posthumes, comme s'ils voulaient dissuader par avance tout biographe ${ }^{47}$ ».

59 A l'ancienne dichotomie livre/télévision, il convient en conséquence d'en substituer une nouvelle, formée de deux couples antithétiques: télévision-auteurs/cinémaécrivains. Voici qui nous reporte, en fin de parcours, aux origines mêmes de notre investigation, puisqu'il nous reste à considérer une question dont l'occurrence même est impliquée par la réponse apportée à cette toute première question pratique: "Comment faire vivre le livre à l'écran quand la lecture y est implicitement tenue pour infigurable? » Très vite, on le sait, les émissions littéraires ont trouvé une réponse devenue incontournable : donner vie à l'écrivain, quitte à en faire un auteur. Comment, dans ces conditions, figurer l'œuvre d'un écrivain qui se dérobe?

Or, cette dernière question qui pourrait passer pour résiduelle, venant alors en quelque sorte prolonger la première jusqu'à l'impasse, s'inscrit au contraire dans une perspective tout autre puisqu'elle ne désigne pas cette fois, dans l'absence de l'écrivain, tant un obstacle à surmonter, qu'une posture indissociable de l'écriture même. Il s'agit donc de réaliser ce qui est proprement la figuration d'une absence. Absence pour ainsi dire «naturelle » dans le cas d'écrivains méconnus de leur vivant et aujourd'hui disparus, mais qu'il faut encore préserver de cette tentation biographique consistant à leur (re)donner vie au moyen d'images d'archives, de documents et autres témoignages. Absence ô combien paradoxale de l'écrivain contemporain, au moment même où il se livre enfin au jeu de l'entretien. Deux modalités de traitement spécifique de cette question de l'absence sont ici annoncées, respectivement mises en œuvre dans les films de la collection Les Hommes-livres ou dans ceux de la collection Préfaces ${ }^{48}$, représentées ici chacune par une réalisation exemplaire. 
61 Le film consacré au poète catalan Salvador Espriu ${ }^{49}$ débute par un bref extrait d'entretien télévisé qui restera d'ailleurs le seul, comme s'il n'en existait pas d'autres : «Je n'ai pas d'aventure personnelle, je suis un homme sans biographie, je suis l'homme le plus effacé de ce monde. J'ai donc très peu à dire sur moi. » Voilà une bien étrange entrée en matière pour un film qui se poursuit en mobilisant toutes les ressources de l'investigation biographique. Coupures de journaux annonçant la mort du poète national, photographies personnelles avec commentaire en voix off: «En 1933, Espriu est en Égypte, il veut être égyptologue ; il ne sait pas encore que c'est le seul voyage de sa vie », documents d'archives sur la guerre d'Espagne, témoignages de ceux qui l'ont connu : Maria Delor éditrice de ses manuscrits, et Félix Cucurull l'ami de toujours. Or, c'est précisément dans l'incongruité même d'une telle mobilisation, s'agissant d'un " homme qui s'est effacé du monde pour maintenir en vie le catalan, interdit pendant quarante ans ", que s'opère ici la figuration de l'absence de biographie de l'écrivain. Espriu en effet ne survit à la guerre que pour « s'enterrer de l'autre côté de la frontière où il est interdit d'écrire, de parler ou d'enseigner le catalan... ». Là, "avec ses souvenirs ressassés à l'infini, il s'agrippe à son crayon pendant quarante ans. devenant le scribe à l'état pur...liant le dernier vers de chaque texte au premier du suivant de sorte qu'un seul poème fasse revivre tous les autres poèmes ». Perdre sa propre biographie n'est plus alors que l'insignifiante contrepartie d'une entreprise visant au sauvetage du sens lui-même. Sauver les mots de la langue pour que vive l'idée d'une nation catalane, voilà ce qu'auront été, tout uniment, la vie et l'œuvre d'Espriu. M. Delor « qui ordonne, commente et transmet l'héritage d'Espriu » en apporte d'abord la confirmation avec cette anecdote : " pendant la guerre, Espriu cachait ses poèmes dans les trous de sa maison pour que si elle s'effondrait sous les bombardements, au moins les mots survivent ». Puis c'est presque au titre de preuve tangible qu'elle présente le fichier où Espriu rassemblait ses notes de lectures, « petite bibliothèque ambulante qu'à tout instant il pouvait sauver ». Le film s'achevant, au surplus, par sa lecture, la voix chargée d'émotion, du poème préféré d'Espriu, «celui qu'il sauverait, disait-il, d'une catastrophe, s'il devait n'en sauver qu'un » : Paroles!

Le film précédent parvenait à figurer l'absence de l'écrivain, entendu comme référent biographique, principe de réalité extérieur à l'œuvre, par le simple déploiement des mêmes techniques documentaires qui l'instituent d'ordinaire comme tel. De la même manière, le film consacré à Louis-René des Forêts, dont l'œuvre est précisément construite autour du malaise inouï de l'expression, et qui a lui-même cessé d'écrire pendant près de vingt ans, parvient pour sa part à thématiser ce silence de l'écrivain à la faveur du même dispositif de l'entretien qui le transforme d'ordinaire en auteur ${ }^{50}$. Loin "d'essayer de briser par des fêlures multiples ce "vœu de silence" 51 ", le film en question vise tout au contraire à le figurer comme tel, à le saisir dans l'instant même de son émergence irrévocable. A preuve, l'entrée en matière vient ici encore problématiser le cadre même dans lequel va s'opérer la découverte de l'écrivain (c'est ici l'entretien en tête-à-tête; c'était la méthode d'investigation documentaire dans le film consacré à Espriu); qu'on en juge !

Plan éloigné d'une demeure, avec une voix inaudible d'abord puis de plus en plus distincte à mesure que la caméra se rapproche de la maison jusqu'à y pénétrer. Deux hommes dans un bureau ; la voix était celle du plus âgé, L.-R. des Forêts, qui poursuit :

L.-R. des F. - Nous sommes tenus par une obligation de parler quand on aimerait rester silencieux.

J.-B. P. - Je ne pense pas qu'on doive s'arrêter à cette considération mais qu'on se 
dise qu'on peut s'arrêter à tout moment.

L.-R. des F.-Oui... Alors arrêtons-nous. de filmer. Et le film lui-même est tout entier constitué de fragments d'entretiens pareils à celui-là, qui ne se tiennent que de ne pouvoir se poursuivre. En fait de tête-à-tête, l'entretien instaure ici une relation à trois dont le principal acteur n'est autre que le réalisateur, dont $\mathrm{M}$. Alphant souligne fort opportunément cette fois la présence invisible : «Benoît Jacquot est si perceptible dans sa façon de filmer l'entretien qu'on sent bien que c'est la réserve même de l'écrivain qui l'intéresse par dessus tout. » Quant à l'interviewer, il s'emploie simplement à amener la conversation sur le terrain du silence, ainsi qu'il l'explique lui-même ${ }^{52}$.

Pour moi, la question essentielle est ici : y a-t-il un rapport entre la période de silence et l'œuvre qui la précède? Compte tenu du fait que cette œuvre évoque presque obsessionnellement l'autodestruction, le mutisme, le silence même, est-ce un « passage à l'acte»?

Ici le Maître résiste. Réponses contradictoires : d'abord il soutient - non sans force effets de manche - qu'il n'y a aucun rapport entre son silence et les œuvres qui précèdent ; puis, plus embarrassé, et face à mon insistance, que s'il y en a un, il n'est pas mieux placé que moi pour en parler : « Vous en revenez toujours au même point pour essayer de m'arracher quelque chose que je ne peux pas vous dire.» Enfin, comme pour faire diversion, il change de sujet et affirme que sa vraie vocation n'est pas la littérature, mais la musique et confie sa passion pour elle. (" J'ai le sentiment complètement illusoire, et un peu fou probablement, que j'aurais pu faire quelque chose en musique que je n'ai jamais pu faire en littérature. Puisque je peux cesser d'écrire, c'est que je ne suis pas un écrivain. ")

La façon dont je pose alors les questions (non sans une certaine audace) et dont L.R. y répond (non sans une simplicité retorse et des réticences ambiguës), nous apparaît presque immédiatement, lors du tournage, comme la répétition involontaire et stupéfiante d'un ou deux récits de L.-R. Nous avons l'impression d'en avoir été malgré nous les interprètes, d'avoir joué devant Benoit Jacquot et son équipe des extraits de Dans un miroir ou des Grands moments d'un chanteur (i.e. dialogue entre un narrateur et un chanteur célèbre qui a renoncé au chant).

Davantage que la posture de l'un ou l'autre, dictée à chacun par sa propre stratégie communicationnelle, c'est proprement l'entretien que filme la réalisateur. Littéralement : l'entretien «chez » L.-R. des Forêts, en tant que figure possiblement (c'est affaire de stratégie communicationnelle) emblématique de son œuvre, comme le type même, tout à la fois, de la conversation contrainte et de la " parole vaine » pour reprendre le titre de la postface ajoutée par Maurice Blanchot au Bavard. Ce que réalise alors véritablement le réalisateur en filmant un tel entretien, c'est le devenir-silence à l'œuvre dans toute expression, dont interviewer et interviewé ne sont que les actants. C'est ainsi qu'il faut entendre cette surprise manifestée par l'interviewer lui-même.

Le plus long silence de l'auteur du Bavard au cours de l'entretien suit le moment où il m'a parlé de son ami prématurément disparu, Jean de Frotté. J'ai été frappé que l'on puisse entendre dans ces propos non seulement une confidence au sujet des rapports entre la vie et l'œuvre de L.-R. des Forêts, mais aussi une description involontaire de notre dialogue lui-même (et peut-être de toute communication, au point de vue de Des Forêts), et des rôles qu'il nous a fait jouer.

On en jugera :

L.-R. des F. - Cet ami essayait de me faire sortir d'une sorte de silence où j'étais, enfin d'une grande réserve... C'est une espèce de lutte entre l'agresseur et l'agressé. 
J.-B. P. - Je me souviens de certains moments de Dans un miroir où deux amis sont confrontés dans une sorte d'entretien, dont la tension d'ailleurs est parfois épuisante.

L.-R. des F. - Oui, c'est ça, c'est exactement ça... ça a un caractère autobiographique, comme toute chose finalement.

J.-B. P. - Mais on retrouve aussi dans une autre nouvelle, un autre récit, dans « La chambre des enfants » même, cette relation extrêmement difficile entre un enfant qui refuse de parler et d'autres enfants qui s'efforcent de lui faire dire une chose qui se dérobe sans cesse.

L.-R. des F. - Oui, il faut croire que c'est un thème qui me hante [silence].

La figuration mimétique de l'œuvre par l'entretien se poursuit. Par un effet de montage, la fin du film thématise l'occurrence même d'un tel entretien. Non seulement le retour à la parole se tient dans l'évocation du retour à l'écriture (i.e. avec Ostinato, dont seuls quelques extraits ont paru en revue), mais la parole recouvrée est tout aussi fragmentaire que l'écriture.

L.-R. des F. - C'est toujours un peu difficile de parler d'un travail en cours ; c'est

même risqué. Donc, peut-être faut-il s'arrêter là.

Avec cette fin de (non) recevoir, voici enfin qu'un écrivain parvient à faire acte de présence à l'écran... en se retirant $! O$ combien édifiant, ce paradoxe met un terme à nos investigations dans le domaine des formes de la présence du livre à la télévision. Et ce, en ramenant la pluralité attendue à une seule et unique modalité, en venant y réunifier «émissions » et «films » dans ce qui apparaît alors comme un corpus homogène ; nous faisant revenir avec la question émergente des conditions d'une telle uniformité, aux prémisses mêmes de nos investigations.

Certes, le film consacré à Louis-René des Forêts, comme du reste celui qui l'était à Salvador Espriu, ainsi que les autres des mêmes collections, remettent bel et bien en cause le statut ontologique de la dite incapacité de l'écrivain à paraître en tant que tel à la télévision. Ils viennent en conséquence contester le monopole télévisuel exercé par ces « auteurs » qu'ont institués les émissions littéraires, figures-repoussoirs déclarées, rappelons-le, de ce genre de réalisations. Mais l'écrivain n'y préserve précisément son intégrité que grâce à la complicité d'un réalisateur, suffisamment habile pour instrumenter en ce sens un dispositif télévisuel visant d'ordinaire à opérer la réduction biographique de l'écrivain et qui apparaît en la circonstance comme tel. Si bien que la parution télévisée de l'écrivain, dans toute son intégrité, requiert paradoxalement pour condition de manifester son altérité radicale avec la télévision, altérité qui du moins n'est plus tant imputable à la nature de l'écrivain qu'à celle du média. En ce sens, et quoi qu'on en dise, ces films extra-ordinaires s'inscrivent dans le même cadre que les émissions ordinaires. De par l'effort consenti et le dispositif mis en place pour y échapper, ils ne font de fait que reconnaître et confirmer la télévision comme instance d'imposition d'une définition biographique de l'écrivain, susceptible de le rendre conforme à cette créature télévisuelle par excellence qu'est l'auteur.

Plus largement, par-delà la remise en cause de la spécificité de ce genre de collections (ouvertement polémiques au demeurant vis-à-vis des émissions littéraires ordinaires, et bénéficiant en vertu de cette prétendue spécificité du soutien des pouvoirs publics), c'est la distinction pourtant élémentaire entre ces deux options alternatives que sont la présentation de livres et le portrait d'écrivain, qui s'estompe à son tour. Inviter le livre, chose inédite mais inerte, implique en effet de recourir à un « auteur » qui puisse à tout le moins lui donner vie. Tout aussi naturellement qu'inviter l'écrivain, être à découvrir 
mais également inerte, implique de concourir à l'événement de sa réalisation biographique. Or, cette nécessité d'animation n'est autre, dans un cas comme dans l'autre, que la contrepartie d'une séparation a priori entre l'écrivain et le livre, et qui ne peut manquer d'apparaitre comme la condition implicite de leur parution respective à l'écran. Comme si la relation primordiale que l'un et l'autre entretiennent, éminemment intime, ne pouvait, comme l'on dit, passer, sur une scène médiatique où c'est au contraire l'espace public qui se met lui-même en représentation... Pas plus du reste que n'est censé passer cet autre modèle de relation intime qu'est supposée être la lecture.

71 Reste que je ne saurais conclure ces investigations littéraro-télévisuelles sans évoquer l'étrange singularité de cette émission de la série Océaniques consacrée au poète roumain Ghérasim Luca. Car c'est bien d'un reste qu'il s'agit, et aux deux sens du terme. D'abord, cette émission est en reste de mes investigations, en cela qu'elle excède le domaine du figurable tel qu'il se constitue sur le double a priori d'une séparation entre l'écrivain et le livre, et d'une incompatibilité entre lecture et télévision. En second lieu, et pour cela même peut-être, elle est ce qu'il m'en reste de plus marquant pour m'avoir tout simplement placé, pour la première fois, en position de téléspectateur impliqué et libéré en quelque sorte de la mauvaise conscience suscitée par une recherche qui me détournait bel et bien de la lecture au profit de la télévision et de son "pis-aller " culturel. Aussi l'aplomb du chercheur doit-il finalement le céder à la stupéfaction du téléspectateur confronté à un poète lisant son livre sans plus. Laissons alors le journal ${ }^{53}$ rendre compte de cet événement et en souligner sa valeur prophétique, laquelle n'est peut-être pas en fin de compte celle que l'on croyait alors.

\section{NOTES}

1. Nous reprenons ici le titre d'un ouvrage pour initiés du linguiste Claude Hagège; ce même ouvrage sur lequel s'étaient ruées des hordes de téléspectateurs après que son auteur en eut magistralement incarné le titre sur le plateau d'Apostrophes.

2. Nos propres comptages portant sur les auteurs invités dans les trois émissions littéraires du premier semestre 1989 donnent une idée d'un tel investissement : pour 50 écrivains déclarés, on retrouve 47 journalistes, 46 enseignants (ou chercheurs), 14 hommes politiques (ou d'affaires), 5 acteurs, 4 aventuriers, 3 prêtres et 2 dessinateurs humoristes.

On trouvera en annexes les tableaux (I bis) de résultats les plus significatifs du second volet de notre étude comparative, portant cette fois sur la part respective des différents genres à la télévision. Pour le premier semestre 1989 : les données concernant les livres passés : à Apostrophes (tab. 1), à Ex-libris (tab. 2), à Libre et change (tab. 3) ; les données concernant les livres passés dans ces trois émissions littéraires (tab. 4). Pour le premier semestre 1980 : les données concernant: les livres passés à Apostrophes (tab. 5), l'ensemble des livres passés à la télévision (tab. 6). Si les grandes tendances sont déjà marquées dès 1980 (en particulier le niveau élevé atteint par les mémoires et autres récits autobiographiques) le tassement du roman s'accuse encore, en partie compensé par la percée de la biographie et des ouvrages de sciences sociales (évolution particulièrement sensible si l'on compare les livres reçus à Apostrophes à dix ans d'intervalle). 
Rappelons à titre complémentaire que sur les 2898 ouvrages passés à Apostrophes entre 1975 et 1986, E. Brasey comptabilise pour sa part 1128 romans $(38,92 \%)$ et 1770 essais, documents (61,08\%). E. Brasey, op. cit., p. 344 . Si ce type de comptage fournit des ordres de grandeur, sa prétention à l'exactitude vient buter sur la question de la définition même des catégories de comptabilisation, question d'autant plus cruciale qu'un des effets du passage du livre à la télévision est précisément de déstabiliser les modes habituels de classement des livres (combien de biographies présentées comme des romans !).

3. Le modèle de présentation adopté par Bernard Pivot pour les auteurs de circonstance invités sur le plateau d'Apostrophes est de ce point de vue remarquable: «Vous êtes professeur (ou journaliste, ou...), et vous publiez (et non pas « vous avez écrit »!)...

4. Les chiffres rapportés ici sont extraits d'une enquête parue dans Le Nouvel Observateur du 15 avril 1988.

5. Le Monde, 28 octobre 1979.

6. N'est-ce pas d'ailleurs à partir d'un présupposé d'identité entre l'«écrivain » et l'«intellectuel» que certains intellectuels se préoccupent aujourd'hui du «chaos dans la république des belles lettres »?

7. Ex-libris, 25 janvier 1989.

8. Apostrophes, 17 février 1989.

9. Ainsi posée, l'opposition auteur-écrivain apparaît comme une reformulation extratextuelle de cette autre opposition entre deux acceptions du style de l'écrivain : comme «transparence » ou comme « obstacle », pour reprendre les fameuses expressions de J. Starobinski.

10. Posée dans ces termes, la question de l'auteur vient s'inscrire dans une problématique plus générale ; elle apparaît comme une manifestation significative de la crise de l'identité dans les sociétés modernes. Mais ceci est une autre histoire.

11. Apostrophes, 4 février 1979.

12. Télérama, 8 février 1989.

13. Télérama, 29 mars 1989, sous le titre "Irène sans frein »!

14. Télérama, 21 septembre 1988.

15. Apostrophes, 27 janvier 1989.

16. Cet intitulé d'une collection de portraits d'écrivains coproduite par la Direction du livre et de la lecture du ministère de la Culture (DLL) nous paraît convenir tout particulièrement pour désigner au contraire l'auteur institué en parangon de son livre par sa propre parution médiatique.

17. Télérama, 28 septembre 1983.

18. Table ronde du 9 avril 1989, deuxièmes rencontres de télévision, Valence, « 25 images/ seconde ».

19. Bernard-Henri Lévy, Éloge des intellectuels, Grasset, 1987.

20. Ex-libris, 18 janvier 1989.

21. Apostrophes, 22 septembre 1978

22. Ex-libris, 22 mars 1989.

23. $C f$. annexes, tableau I bis.

24. Apostrophes, 7 avril 1989.

25. J. Starobinski, L'CEil vivant, tome 2, Gallimard, 1971, p. 86.

26. Apostrophes, 3 mars 1989.

27. Apostrophes, 3 mars 1989.

28. Apostrophes, 20 janvier 1989

29. Ibid.

30. Rappelons que de l'aveu même de Bernard Pivot, une émission comme Apostrophes ne doit pas être regardée comme une émission de critique littéraire ; ce à quoi la formule de l'émission ne se 
prête du reste pas : peu de temps imparti à la présentation de chaque ouvrage, difficulté pour des auteurs juge et partie à s'accorder selon le critère du beau.

31. Au terme duquel l'identité sociale ne consiste plus que dans un seul attribut. Dès lors le «stigmate » occupe toute la place; il sature l'espace d'ordinaire ouvert à la présentation de soi. Sur ce point, $c f$. E. Goffman, Stigmates, Éd. de Minuit, 1975.

32. Apostrophes, 10 mars 1989.

33. Apostrophes, 24 février 1989.

34. Lequel peut finir par se persuader qu'il a lui-même écrit le livre et se faire un plaisir de raconter ses affres d'écrivain... pour peu qu'il ait pris la peine de réécrire, de sa propre main, le manuscrit et qu'il participe ensuite à un nombre suffisant de séances de signatures en librairie ou dans les diverses fêtes du livre. Tel est le cas connu du Tout-Paris littéraire de cet auteur à succès invité pourtant à Apostrophes alors même que P.-L. Sulitzer y est ostensiblement interdit de séjour ! Comme s'il fallait faire payer à celui qui pratique ouvertement cela même que l'autre n'a de cesse de cacher, d'avoir bafoué le mythe de l'écrivain.

35. Cf. Livres-Hebdo, 17 avril 1989, « Le grand écran pour Sulitzer ».

36. Ex-libris, 5 avril 1989.

37. Apostrophes.

38. Apostrophes, 7 décembre 1979.

39. Apostrophes, 28 septembre 1984.

40. Notons, à ce propos, que le passage à Apostrophes de Marguerite Yourcenar venait également, quoique dans une moindre mesure, ponctuer une inflexion de son œuvre vers une manière d'autogénéalogie.

41. J. Lecarme, B. Vercier : «Premières personnes », Le Débat, $\mathrm{n}^{\circ}$ 54, 1989.

42. Dans la mesure où, comme le remarque E. Goffman, une information est tenue pour d'autant plus fiable qu'elle ne paraît pas entièrement contrôlée par celui qui la livre. Cf. La Mise en scène de la vie quotidienne, tome 1, Éd. de Minuit, 1973, p. 17.

43. Portraits d'auteurs, brochure de la présentation des films soutenus par la DLL, 1989.

44. Ibid.

45. Interview exclusive.

46. Rappelons que Marguerite Duras tout comme Marguerite Yourcenar passent à Apostrophes après être passées de quelque manière à l'autobiographie.

47. C. Arnaud, «Le retour de la biographie », Le Débat, $\mathrm{n}^{\circ}$ 54, 1989.

48. Voici les textes de présentation de ces deux collections tels qu'ils figurent dans la brochure du ministère de la Culture déjà citée. Les Hommes-livres : collection produite par l'Ina, la Sept, avec la participation de la Direction du livre et de la lecture. Portraits d'écrivains français ou de langue française, auteurs vivants, parvenus à la plénitude de leur œuvre mais qui, jusqu'à présent, ont été méconnus, notamment par la télévision, soit qu'elle les ait ignorés, soit qu'ils s'en soient délibérément écartés. Chaque film, réalisé dans l'un des lieux naturels de la vie de l'écrivain, est un entretien filmé pendant un laps de temps assez ramassé. Par la manière de filmer et la mise en scène de l'entretien, ces portraits cherchent à faire écho à l'œuvre ellemême, à inviter à la lecture. Préfaces : collection produite par Archipel 33, la Sept, avec la participation de la Direction du livre et de la lecture. «A l'origine de cette série d'essais, il y a le désir d'introduire à la lecture d'œuvres d'écrivains européens du $\mathrm{xx}^{\mathrm{e}}$ siècle aujourd'hui disparus. Longtemps, leur existence ne fut connue que de rares amateurs et leur œuvre ne franchit qu'exceptionnellement les limites de leur pays. L'ombre des "géants", Kafka, Proust, Joyce... les aurait conduits à l'oubli s'il n'y avait eu l'obstination passionnée de premiers lecteurs qui ont perpétué leurs noms jusqu'à ce que rééditions et traductions élargissent le cercle. Aujourd'hui, ils s'imposent, chacun à sa façon, comme des auteurs essentiels. La forme : un lecteur-réalisateur, qui a déjà lu, s'adresse à des spectateurs-lecteurs, qui n'ont pas encore lu. Pas d'exégèse ou d'explication, pas d'extraits de films adpatés des œuvres, rien qui puisse se substituer à la lecture. 
Chaque film a le caractère d'une préface personnelle » (Pierre Beuchot, note d'intention, février 1988).

49. Salvador Espriu, film d'Henri Colomer, 1989, collection Préfaces.

50. Louis-René des Forêts, film de Benoît Jacquot, entretien Jean-Benoît Puech, 1988, collection Les Hommes-livres.

51. Comme le prétend Marianne Alphant dans un article de présentation par ailleurs remarquable : «Le silence des forêts », Libération, 21 mars 1988.

52. "A propos de l'entretien », par J.-B. Puech. Texte figurant dans le livret de présentation du film. Nous avons rajouté entre parenthèses les fragments d'entretien dont il est question.

53. Cf. Le Monde, supplément télévision, 18-19 février 1989, p. 7. 


\section{Conclusion}

1 A l'origine de nos investigations nous avons pris appui sur la problématisation ordinaire du rapport livre-télévision et son interminable querelle des inférences pour en souligner le caractère aporétique. Prétendre évaluer l'efficacité de la télévision sur la lecture présuppose à tout le moins une stabilité des termes du rapport et en particulier de la lecture dont la constance n'est autre en fait que celle assurée par l'hégémonie d'un modèle de lecture cultivée. En établissant que la télévision opère au contraire des transformations profondes dans la signification même de la lecture, la suite de nos investigations devait confirmer la problématisation ordinaire du rapport livre-télevision en sa fonction instituante, comme opération de construction sociale d'une réalité des pratiques et de leur rapport, conforme au modèle de la culture savante. Par le fait même de leur mise en rapport, la lecture cesse d'être une pratique parmi d'autres pour devenir valeur puisque son propre niveau sert d'étalon de mesure universel (i.e. pour mesurer les autres pratiques), tandis qu'inversement la télévision ne fait jamais que confirmer l'hégémonie de la lecture quelle qu'en soit au demeurant l'incidence sur son niveau! Cela établi et alors seulement on peut appréhender l'efficacité sociale implicite de la problématisation ordinaire du rapport livretélévision : assurer une neutralisation symbolique de la télévision, au moment même où celle-ci opère une transformation substantielle de la lecture.

On saisira toute l'ampleur et le sens de cette transformation si l'on se réfère au modèle de l'immédiateté du rapport entre l'écrivain et son lecteur ou, si l'on préfère, de la coïncidence entre la réception de l'œuvre et la lecture du livre. Modèle défini conjointement par les théories de l'acte de lecture et de l'esthétique de la réception ${ }^{1}$ qui constituent le seul véritable précédent en matière d'analyse de la lecture proprement dite. Par conséquent, et même s'il est d'origine extra-disciplinaire, leur modèle théorique est un point d'appui obligé pour qui entend poser les jalons d'une approche compréhensive de l'acte de lecture, que ne peut décidément continuer d'ignorer la sociologie de la lecture ${ }^{2}$. Rappelons brièvement que d'après la théorie de $\mathrm{W}$. Iser, chaque texte présuppose un lecteur: il implique une sorte de position vide que le lecteur concret va occuper lors de sa lecture. Tout le texte s'organise alors autour de cette position de "lecteur implicite». Il représente une structure de virtualité qui s'actualise dans la lecture concrète par le lecteur réel prenant la position du lecteur implicite. Or voici que la médiatisation de l'auteur suscite un réel extra-textuel 
intermédiaire entre mondes du texte et de l'auteur ; voici donc que l'immédiateté du rapport à l'auteur tient lieu d'immédiateté du rapport au texte, voici enfin que la réception télévisée précède la lecture et peut même en tenir lieu.

Dans la transformation à l'œuvre, on peut ainsi voir un dessaisissement de l'auteur des dispositifs de gestion de l'entrée du lecteur dans son texte. Pour notre part, nous y voyons plutôt un élargissement de la fonction-auteur : à travers ce que dit l'auteur de son livre sur les différentes scènes médiatiques, il s'agirait pour lui de déployer une stratégie non plus textuelle mais extra-textuelle. Nous touchons ici aux limites inhérentes au cadre même de notre réflexion ; l'orientation de recherche qui se dessine requérant en effet un protocole d'observation transversal au nôtre, dont l'unité ne serait plus tant l'émission - ses éditions successives - que l'auteur - la série de ses prestations sur les différentes scènes médiatiques ${ }^{3}$. Mentionnons seulement ce cas exemplaire du travail de gestion de la parole publique que l'auteur doit accomplir aujourd'hui.

4 Madeleine Chapsal, on s'en souvient, fut sommée publiquement par l'animateur d'Apostrophes d'avouer que l'histoire narrée dans son dernier roman, La Maison de jade (Grasset) - une femme délaissée par son amant beaucoup plus jeune qu'elle - était entièrement autobiographique. Résultat: La Maison de jade est le best-seller de sa carrière. Deux mois après, Madeleine Chapsal passe à la radio, interrogée par Jacques Chancel dans Singulier-pluriel (France-Inter, le 29 janvier 1987). Là, plus question de nier l'autobiographie ; elle est à l'inverse, rappelée avec insistance ("J'ai mis longtemps à m'en sortir ", "J'espère aider les femmes qui ont été dans le même cas que moi », "Oui, j'espère être de nouveau amoureuse $\left.{ }^{4} »\right)$.

5 Il ne faudrait pas croire non plus que le travail de l'écriture et la gestion de la parole publique soient des activités contradictoires, la première mettant en intrigue, ménageant des virtualités, autorisant à des lectures; là où la seconde impose une lecture et une seule, au demeurant le plus souvent caricaturale. La fortune récente du récit autobiographique ${ }^{5}$ suffit à témoigner du contraire puisque, dans ce cas, le texte dans le livre et la présentation de soi sur le plateau ne sont jamais que les deux facettes d'un seul et même dispositif autodiégétique.

6 Qu'est-ce encore que la lecture, sera-t-on tenté de se demander, dès lors qu'elle n'est plus cette heureuse rencontre entre « le monde du texte » et le « monde du lecteur ${ }^{6}$ ». Il est sans doute prématuré de poser aujourd'hui une question aussi radicale quoique, selon nous, elle n'en constitue pas moins dès à présent l'horizon des recherches à venir dans le domaine de la sociologie de la lecture. Pour l'heure, il s'agit simplement de reconnaître que l'« esthétique de la réception", dans le domaine de la sociologie idéalise cette rencontre qu'est la lecture en la désocialisant; et que parmi ces médiations autorisant voire facilitant l'accès au texte, qu'elle occulte, il en est une nouvelle, l'auteur lui-même. En d'autres termes, si nos investigations n'invalident pas de manière définitive cette représentation de la lecture doublement hégémonique (la théorie de 1'«esthétique de la réception » reproduisant dans le champ scientifique la position dominante qu'occupe déjà sur le terrain des pratiques la même représentation de la lecture comme expérience lettrée), du moins permettent-elles de la socialiser par l'intermédiaire d'une notion dérivée de celle de "paratexte ». La notion de paratexte, empruntée à G. Genette ${ }^{7}$, vise, rappelons-le, à désigner l'ensemble de ce qui dans le livre constitue l'environnement du texte proprement dit (titre, préface, avertissement, 
illustrations, etc.) et dont l'effet, souvent méconnu, concerne la possibilité d'établissement du contrat générique (P. Lejeune) entre l'auteur et le lecteur.

7 Avec le livre à la télévision, force est d'élargir cette notion de paratexte. Alors en effet, celui qui regarde pour se faire une idée, a affaire, non plus seulement à l'écrivain, producteur d'énoncés textuels, mais à l'interlocuteur, producteur, avec plus ou moins de facilité, d'énonciations, et plus encore au personnage, son visage, ses attitudes, sa prestance.

8 Une dimension nouvelle apparaît qui au lieu d'être contenue dans le seul paratexte - ce qui est dans le livre et pas dans le «texte » - se disperse plutôt dans la multiplication des scènes mises en place autour du livre. Aussi, au lieu que dans la théorie de la réception (du texte) l'écrivain est conçu comme travaillant à construire le monde intérieur d'un lecteur implicite, le professionnel des scènes du «paralivre » est celui qui travaille à la visibilité du livre pour des récepteurs implicites. Son objectif, dont il est dit qu'il a la lecture pour horizon, s'actualise en fait dans une série de dispositifs visant toutes les autres formes d'appropriation -audiovisuelles- que celle réalisée par la lecture ("J'l'ai pas lu, mais j'I'ai vu ou j'en ai entendu causer »).

D'ailleurs, le livre même se transforme, sous le régime médiatique du "paralivre »: il tend à n'être plus l'objet d'une pratique autonome, à ne plus renfermer un monde du texte spécifique pour devenir un univers familier ; n'engageant plus à la découverte, il sollicite les retrouvailles ${ }^{8}$.

Voilà déjà fort longtemps, objectera-t-on, que le livre se met en scène: avec sa couverture, première et quatrième, la bande-annonce, le format et le logo de l'éditeur ou de la collection, etc. Cependant il ne s'agissait là que du livre lui-même en tant qu'objet, et la mise en scène restait limitée au domaine du paratexte. On rentre par contre dans l'univers infini du paralivre avec le présentoir chez le libraire, le placard publicitaire dans la presse écrite, l'affiche dans le métro, la séance de signature, la présence de l'auteur à telle ou telle fête du livre. Toutes manifestations de l'existence sociale du livre détachées de l'objet-livre et partant de la lecture. Ainsi définis, les territoires du paralivre dans leur profusion même sont autant d'annexes de la scène télévisuelle. Quand ils n'en sont pas directement le relais (le cas le plus caricatural étant sans doute celui des «fêtes du livre » où le public peut retrouver les auteurs tels qu'en eux-mêmes ${ }^{9}$ ), ils témoignent d'une volonté d'échapper à l'effet d'imposition télévisuelle ${ }^{10}$. Sur le terrain de la pratique des professionnels du livre tout au moins nous sommes d'ores et déjà bien loin de la querelle de l'inférence.

\section{NOTES}

1. W. Iser, L'Acte de lecture, Mardaga, 1985. J.-R. Jauss, Pour une esthétique de la réception, Gallimard, 1973.

2. Sur ce point, $c f$. Michel Peroni, Histoires de lire, op. cit.

3. On comprendra l'impossibilité où nous sommes d'aller plus loin en ce sens si l'on sait que les diverses émissions littéraires demandent souvent l'exclusivité à leurs invités. 
4. « Apostrophes, $1357^{\mathrm{e}}$ édition? ?, E. Brasey, Journal du salon, 1987.

5. Telle que la plupart des écrivains des avant-gardes d'hier ont fini par s'y résoudre, $c f . J$. Lecarme, B. Vercier, « Premières personnes », Le Débat, n 54, 1989.

6. Nous empruntons ces deux expressions à Paul Ricœur, Temps et récit, tome 3, Le Temps raconté, chapitre v, « Monde du texte et monde du lecteur », Éd. du Seuil, 1955.

7. Cf. Seuils, Éd. du Seuil, 1986.

8. Nombreuses et diverses sont les formes d'une telle transformation. En premier lieu il faut signaler la multiplication de ce que l'on peut appeler les «livres d'émission »; de plus en plus nombreuses sont les séries télévisées achetées par les éditeurs pour devenir des livres. Certains comme Menges et Sylvie Mes-singer sont spécialisés dans ce type de productions, baptisées du nom anglais de novelisation («Livre/télévision: des relations de plus en plus étroites ", LivresHebdo, 23 juin 1981), TF1-Éditions, créé au printemps 1988, assure pour sa part les publications dérivées d'émissions de la chaîne ou d'auteurs qui y travaillent. Plus original : « depuis quelques années, livre et télévision tentent de travailler la main dans la main en coproduisant d'emblée livre et émission nés d'un projet commun » ("Le livre et la télévision : un intérêt bien compris », Livres-Hebdo, 13 octobre 1986). Notons en second lieu l'apparition de nouveaux types de supportlivre, tels le livre-caméra que Jean-Marie Drot a tiré de son émission de télévision sur l'écrivain Joseph Delteil (Bibliographie de la France, $\mathrm{n}^{\circ} 21,1976$ ). N'oublions pas enfin la prolifération de fastbooks, ouvrant largement le monde du livre sur les vedettes et les événements de l'actualité médiatique. Décidément, entre le livre et la télévision, l'heure n'est plus à la concurrence mais bel et bien à l'intégration.

9. Souvenir de la fête du livre de Saint-Etienne : deux "pépés » immobiles dévisageant une belle femme rousse qui signe à tour de bras. Elle est installée de l'autre côte d'une impressionnante pile de livres titrés "Régine Deforges", elle se tient sous un panneau marqué "Régine Deforges ». L'un des pépés dit à l'autre: "Tu la reconnais, c'est "la" Deforges, on l'a vue à la télé ». « Mais c'est pas un écrivain ça », répond l'autre qui ajoute, en désignant une place restée vide au même stand derrière une pile titrée «Jean-Edern Hallier » et sous une pancarte marquée «Jean-Edern Hallier » : « Ça, c'est un écrivain! Pas étonnant qu'il n'y soit pas ».

10. C'est ainsi que les libraires ne peuvent aujourd'hui préserver leur fonction de conseil qu'à la condition de « redéfinir leur compétence en amont de l'effet télévisuel » (entretien Asfodelp). 


\section{Annexes}

1 Une étude financée par la Délégation du livre et de la lecture du ministère de la Culture est à l'origine de ce livre. Elle a fait l'objet d'un rapport dans lequel figurent des annexes complémentaires; il peut être consulté au service des études et de la recherche de la Bibliothèque publique d'information du Centre Georges Pompidou.

Tableau A. Lecture et possession de la télévision

Depuis que vous avez la télévision, lisez-vous plus, autant ou moins?

\begin{tabular}{|l|r|c|c|}
\hline & Plus & Moins & Autant \\
\hline De magazines illustrés & $5 \%$ & $56 \%$ & $39 \%$ \\
De romans & $4 \%$ & $59 \%$ & $38 \%$ \\
De livres documentaires & $14 \%$ & $49 \%$ & $37 \%$ \\
De quotidiens & $4 \%$ & $42 \%$ & $52 \%$ \\
\hline
\end{tabular}

Source : IRES. 
Tableau B : Possession de livres, audience des émissions littéraires et lecture de revues spécialisées

\begin{tabular}{|c|c|c|c|c|c|c|}
\hline & \multicolumn{6}{|c|}{ Sur 100 personnes de chaque groupe } \\
\hline & \multirow{2}{*}{$\begin{array}{c}\text { Ensemble de la } \\
\text { population étudiée }\end{array}$} & \multicolumn{5}{|c|}{ Nombre de livres possédés } \\
\hline & & Aucun & 1 à 25 & 26 à 99 & 100 à 250 & Plus de 250 \\
\hline & $\%$ & $\%$ & $\%$ & $\%$ & $\%$ & $\%$ \\
\hline $\begin{array}{l}\text { Possèdent la télévision et regardent } \\
\text { les émissions sur la littérature et les } \\
\text { écrivains: } \\
\text { - souvent } \\
\text { - de temps en temps } \\
\text { - rarement } \\
\text { - jamais } \\
\text { - Ne se prononcent pas } \\
\text { - Ne possèdent pas la télévision }\end{array}$ & $\begin{array}{r}9,2 \\
16,5 \\
16,1 \\
43,8 \\
0,3 \\
14,1 \\
\end{array}$ & $\begin{array}{r}4,3 \\
6,6 \\
9,5 \\
60,0 \\
0,5 \\
19,0 \\
\end{array}$ & $\begin{array}{r}4,9 \\
10,6 \\
18,0 \\
53,3 \\
1,0 \\
12,2 \\
\end{array}$ & $\begin{array}{r}6,9 \\
20,2 \\
19,6 \\
41,9 \\
0,1 \\
11,3 \\
\end{array}$ & $\begin{array}{r}15,0 \\
24,3 \\
16,9 \\
31,2 \\
- \\
12,7\end{array}$ & $\begin{array}{r}19,0 \\
24,8 \\
19,7 \\
22,4 \\
0,2 \\
13,3 \\
\end{array}$ \\
\hline & 100,0 & 100,0 & 100,0 & 100,0 & 100,0 & 100,0 \\
\hline $\begin{array}{l}\text { Lecture d'une revue spécialisée } \\
\text { (littéraire, artistique, scientifique, } \\
\text { d'histoire, etc.): } \\
\text { - souvent } \\
\text { - de temps en temps } \\
\text { - rarement } \\
\text { - jamais } \\
\text { - Ne se prononcent pas }\end{array}$ & $\begin{array}{r}12,2 \\
10,5 \\
6,6 \\
70,0 \\
0,8\end{array}$ & $\begin{array}{r}2,6 \\
1,7 \\
1,4 \\
92,3 \\
2,0\end{array}$ & $\begin{array}{r}4,7 \\
9,2 \\
5,7 \\
79,4 \\
1,0\end{array}$ & $\begin{array}{r}10,7 \\
10,0 \\
10,3 \\
68,7 \\
0,3\end{array}$ & $\begin{array}{r}17,8 \\
18,2 \\
9,5 \\
54,5 \\
-\end{array}$ & $\begin{array}{r}35,4 \\
18,2 \\
7,2 \\
39,2 \\
- \\
\end{array}$ \\
\hline & 100,0 & 100,0 & 100,0 & 100,0 & 100,0 & 100,0 \\
\hline
\end{tabular}

Source : Les Pratiques culturelles des Français, 1973.

Tableau C. Possession de livres et présence de biens culturels

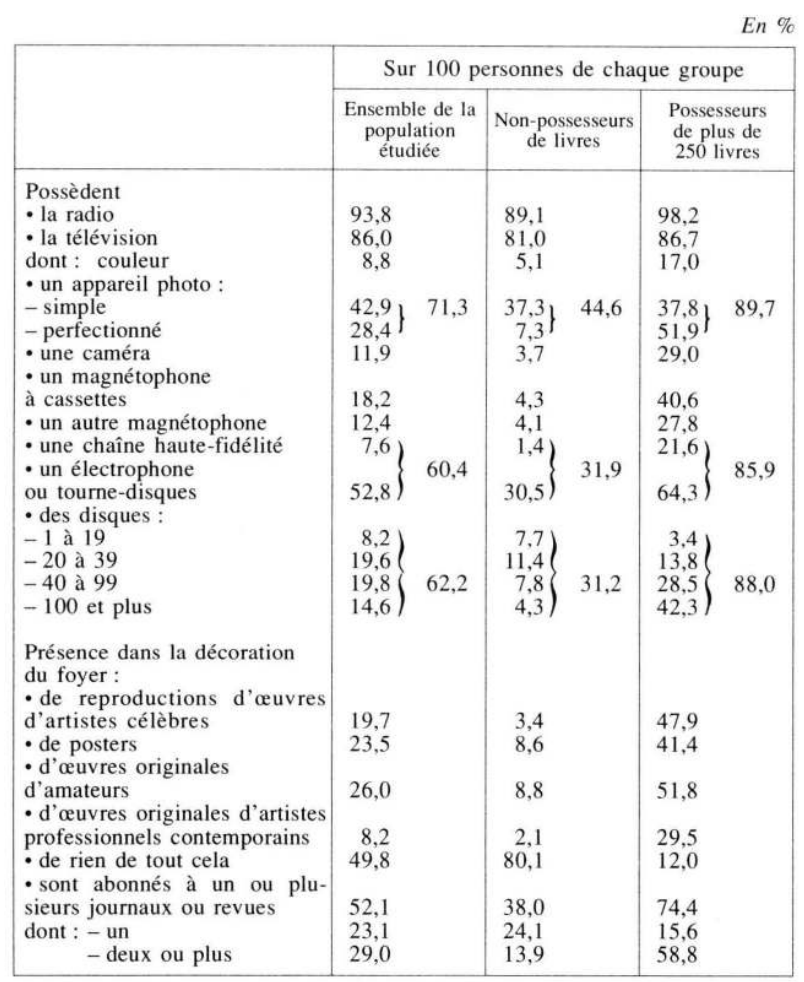

Source : Les Pratiques culturelles des Français, 1973 
Tableau D. Nombre de livres lus par an et assiduité aux émissions littéraires en 1973 et 1981

\begin{tabular}{|c|c|c|c|c|c|c|c|}
\hline \multirow[b]{2}{*}{$\begin{array}{l}\text { Nombre de livres } \\
\text { lus depuis } 1 \text { an }\end{array}$} & \multicolumn{3}{|c|}{1973} & \multicolumn{3}{|c|}{1981} & \multirow{2}{*}{ 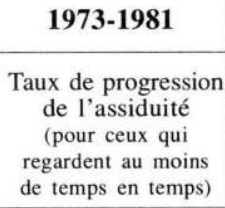 } \\
\hline & $\begin{array}{c}\text { Regardent } \\
\text { souvent }\end{array}$ & $\begin{array}{l}\text { De temps } \\
\text { en temps }\end{array}$ & $\begin{array}{c}\text { Au } \\
\text { moins } \\
\text { de temps } \\
\text { en temps }\end{array}$ & $\begin{array}{c}\text { Regardent } \\
\text { souvent }\end{array}$ & $\begin{array}{l}\text { De temps } \\
\text { en temps }\end{array}$ & $\begin{array}{c}\text { Au } \\
\text { moins } \\
\text { de temps } \\
\text { en temps }\end{array}$ & \\
\hline Ensemble & 11,6 & 19,1 & 30,7 & 14,3 & 21,0 & 35,4 & \\
\hline De 1 à 9 & 8,4 & 21,0 & 29,4 & 13,3 & 21,3 & 34,6 & $+17,7 \%$ \\
\hline De 10 à 24 & 15,4 & 25,1 & 40,5 & 29,2 & 28,0 & 49,0 & $+21,2 \%$ \\
\hline De 25 à 49 & 19,6 & 27,8 & 47,4 & 24,2 & 31,0 & 55,2 & $+16,4 \%$ \\
\hline 50 et plus & 18,0 & 25,4 & 43,4 & 22,6 & 23,7 & 46,3 & $+\quad 6,7 \%$ \\
\hline Aucun & 3,3 & 8,1 & 11,4 & 4,2 & 9,3 & 13,4 & $+17,6 \%$ \\
\hline
\end{tabular}

Ensemble des possesseurs de télévision $=100 \%$

Source : Les Pratiques culturelles des Français, 1973 et 1981

Tableau E. Le public des émissions littéraires

\begin{tabular}{|c|c|c|}
\hline & Italiques & $\begin{array}{l}\text { Échantillon } \\
\text { du panel }\end{array}$ \\
\hline $\begin{array}{l}\text { Sexe } \\
\text { Hommes } \\
\text { Femmes }\end{array}$ & $\begin{array}{l}39 \\
61\end{array}$ & $\begin{array}{l}47 \\
53\end{array}$ \\
\hline $\begin{array}{l}\text { Age } \\
15 \text { à } 19 \text { ans } \\
20 \text { à } 24 \text { ans } \\
25 \text { à } 34 \text { ans } \\
35 \text { à } 49 \text { ans } \\
50 \text { à } 64 \text { ans } \\
65 \text { ans et plus }\end{array}$ & $\begin{array}{r}1 \\
3 \\
15 \\
25 \\
30 \\
25\end{array}$ & $\begin{array}{l}12 \\
10 \\
15 \\
30 \\
20 \\
13\end{array}$ \\
\hline $\begin{array}{l}\text { Niveau d'instruction } \\
\text { primaire } \\
\text { primaire supérieur } \\
\text { technique-commercial } \\
\text { secondaire } \\
\text { supérieur }\end{array}$ & $\begin{array}{l}39 \\
12 \\
10 \\
18 \\
21\end{array}$ & $\begin{array}{r}48 \\
8 \\
15 \\
21 \\
8\end{array}$ \\
\hline $\begin{array}{l}\text { Profession de la personne } \\
\text { agriculteurs } \\
\text { commerçants, industriels } \\
\text { cadres supérieurs } \\
\text { cadres moyens } \\
\text { employés } \\
\text { ouvriers } \\
\text { divers actifs } \\
\text { élèves, étudiants } \\
\text { retraités } \\
\text { ménagères et autres inactifs }\end{array}$ & $\begin{array}{r}1 \\
2 \\
10 \\
11 \\
7 \\
13 \\
13 \\
0 \\
1 \\
18 \\
38\end{array}$ & $\begin{array}{r}5 \\
3 \\
5 \\
8 \\
11 \\
17 \\
1 \\
9 \\
12 \\
29\end{array}$ \\
\hline $\begin{array}{l}\text { Catégories de communes } \\
\text { communes rurales } \\
\text { villes de }- \text { de } 100000 \text { hab. } \\
\text { villes de + de } 100000 \text { hab. } \\
\text { Paris }\end{array}$ & $\begin{array}{l}13 \\
24 \\
26 \\
38\end{array}$ & $\begin{array}{l}24 \\
30 \\
28 \\
18\end{array}$ \\
\hline Ensemble & 100 & 100 \\
\hline
\end{tabular}

Source : SEO, 1972. 
Tableau F. Achat de livres et fréquence d'écoute des émissions littéraires

\begin{tabular}{|c|c|c|c|c|c|}
\hline & Au cours & six derniers moi & ont acheté... & \multirow{3}{*}{ NSP } & \multirow{3}{*}{$\begin{array}{c}\text { Effectifs } \\
100 \%\end{array}$} \\
\hline & un livre & plusieurs livres & aucun livre & & \\
\hline & \multicolumn{3}{|c|}{$\begin{array}{l}\text { dont ils ont entendu parler dans une émission } \\
\text { de télévision sur les livres }\end{array}$} & & \\
\hline $\begin{array}{l}\text { Parmi ceux qui regardent les émis- } \\
\text { sions de télévision sur les livres: }\end{array}$ & & & & & \\
\hline $\begin{array}{l}\text { souvent } \\
\text { de temps en temps } \\
\text { rarement }\end{array}$ & $\begin{array}{r}17,2 \% \\
14,6 \% \\
4,8 \%\end{array}$ & $\begin{array}{r}34,7 \\
12,2 \\
1,9 \\
\end{array}$ & $\begin{array}{l}44,5 \\
70,9 \\
91,6\end{array}$ & $\begin{array}{l}3,6 \\
2,2 \\
1,7\end{array}$ & $\begin{array}{l}159 \\
235 \\
235\end{array}$ \\
\hline Ensemble & $11,6 \%$ & 14,1 & 71,9 & 2,4 & 629 \\
\hline
\end{tabular}

Source : CEO, 1984.

Tableau G. Lecture ou écoute d'informations sur les livres, et fréquence de l'usage des différents médias

En \%

\begin{tabular}{|l|c|c|c|c|}
\hline & $\begin{array}{c}\text { Journal } \\
\text { quotidien }\end{array}$ & $\begin{array}{c}\text { Revues et } \\
\text { magazines }\end{array}$ & Télévision & Radio \\
\cline { 2 - 5 } (A) : Lisent, écoutent & & & & \\
au moins une fois par semaine & 75 & 56 & 91 & 87 \\
(B) : au moins une fois par mois & 80 & 81 & 93 & 90 \\
& & & & \\
Lisent, écoutent des informations & & & & \\
sur les livres : & & & & \\
(a) : au moins une fois par semaine & 20 & 27 & 27 & 16 \\
(b) : au moins une fois par mois & 37 & 45 & 46 & 27 \\
\hline a/A = & 0,35 & 0,48 & 0,30 & 0,18 \\
b/B $=$ & 0,46 & 0,56 & 0,50 & 0,30 \\
\hline
\end{tabular}

Source : ARC-mc 


\begin{tabular}{|l|c|}
\hline \multicolumn{2}{|l|}{ En \% } \\
\hline $\begin{array}{l}\text { a) après avoir lu, dans un journal ou une revue, } \\
\text { un article qui vous avait signalé ce livre }\end{array}$ & 37 \\
$\begin{array}{l}\text { b) après avoir entendu parler de ce livre } \\
\text { dans une émission de télévision }\end{array}$ & 30 \\
$\begin{array}{l}\text { c) après avoir entendu parler de ce livre } \\
\text { dans un émission de radio }\end{array}$ & 17 \\
$\begin{array}{l}\text { d) après avoir remarqué une publicité } \\
\text { en faveur de ce livre dans la presse ou à la radio }\end{array}$ & 26 \\
$\begin{array}{l}\text { e) après avoir vu un film de cinéma ou de télévision } \\
\text { tiré de ce livre }\end{array}$ & 27 \\
$\begin{array}{l}\text { f) après avoir lu ce livre ou une partie de ce livre } \\
\text { en feuilleton, en roman-photo ou en bandes dessinées }\end{array}$ & 13 \\
g) après avoir suivi un jeu télévisé & 3 \\
\hline
\end{tabular}

Source : ARC.mc 
Tableau I Intervalle entre la date de publication des livres et celle de leur passage à l'antenne

1989

\begin{tabular}{|c|c|}
\hline \multicolumn{2}{|c|}{ Apostrophes } \\
\hline 1 & 24,1 \\
\hline 2 & 15,3 \\
\hline 3 & 25,8 \\
\hline 4 & 18,5 \\
\hline 5 & 12,0 \\
\hline 6 & 4,0 \\
\hline 7 & - \\
\hline
\end{tabular}

Effectif : 124

Tableau 1

\begin{tabular}{|c|r|}
\hline \multicolumn{2}{|c|}{ Ex-libris } \\
\hline 1 & 34,1 \\
\hline 2 & 8,9 \\
\hline 3 & 19,5 \\
\hline 4 & 16,2 \\
\hline 5 & 9,7 \\
\hline 6 & 10,5 \\
\hline 7 & 0,8 \\
\hline
\end{tabular}

Tableau 2

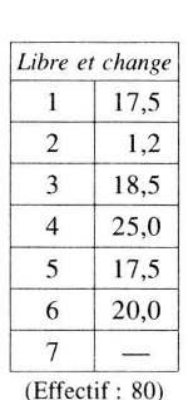

Tableau 3

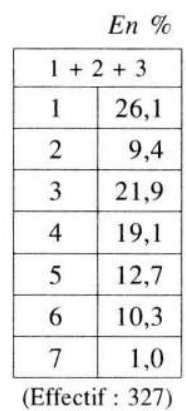

Tableau 4

\section{0}

\begin{tabular}{|c|c|}
\hline \multicolumn{2}{|c|}{ Apostrophes } \\
\hline 1 & 35,6 \\
\hline 2 & 12,8 \\
\hline 3 & 14,8 \\
\hline 4 & 20,7 \\
\hline 5 & 7,9 \\
\hline 6 & 7,9 \\
\hline 7 & - \\
\hline
\end{tabular}

Tableau 5

\begin{tabular}{|c|c|}
\hline \multicolumn{2}{|c|}{$\begin{array}{c}\text { Émissions } \\
\text { littéraires }\end{array}$} \\
\hline 1 & 29,6 \\
\hline 2 & 14,2 \\
\hline 3 & 16,6 \\
\hline 4 & 26,5 \\
\hline 5 & 6,8 \\
\hline 6 & 6,1 \\
\hline 7 & - \\
\hline
\end{tabular}
(Effectif : 162 )

Tableau 6

\begin{tabular}{|c|r|}
\hline \multicolumn{2}{|c|}{ Télévision } \\
\hline 1 & 26,2 \\
\hline 2 & 11,0 \\
\hline 3 & 14,6 \\
\hline 4 & 25,0 \\
\hline 5 & 6,3 \\
\hline 6 & 14,1 \\
\hline 71 & 2,0 \\
\hline
\end{tabular}

Tableau 7

\begin{tabular}{|l|r|}
\hline \multicolumn{2}{|c|}{$\begin{array}{l}\text { Journal } \\
\text { télévisé }\end{array}$} \\
\hline 1 & 23,4 \\
\hline 2 & 8,1 \\
\hline 3 & 12,2 \\
\hline 4 & 16,3 \\
\hline 5 & 10,2 \\
\hline 6 & 20,4 \\
\hline 6 & 9,1 \\
\hline
\end{tabular}

Tableau 8

\begin{tabular}{|c|c|}
\hline \multicolumn{2}{|c|}{$\begin{array}{c}\text { Plus d'une } \\
\text { fois TV }\end{array}$} \\
\hline 1 & 20,5 \\
\hline 2 & 19,1 \\
\hline 3 & 23,5 \\
\hline 4 & 26,4 \\
\hline 5 & 5,9 \\
\hline 6 & 1,4 \\
\hline 7 & - \\
\hline \multicolumn{2}{|c|}{ (Effectif : 68 ) }
\end{tabular}

Tableau 9

Codification

1. Livres passés avant publication

2. Livres passés dans la semaine de leur publication

3. Plus d'une semaine et moins d'un mois

4. Plus d'un mois et moins de trois mois

5. Plus de trois mois et moins de six mois

6. Plus de 6 mois

7. Livres d'auteurs « classiques» 
Tableau I bis : Les différentes catégories de livres présentées à la télévision

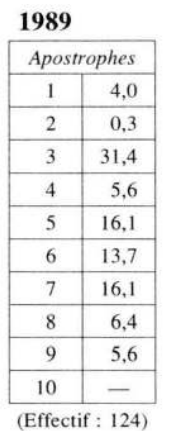

Tableau 1

1980

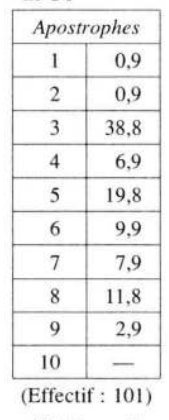

Tableau 5
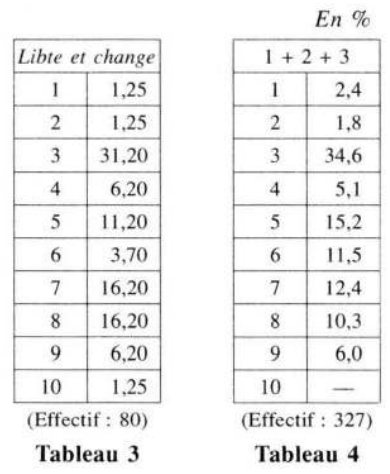

Tableau 4

Codification

1. Littérature générale

2. Poésie

3. Roman

4. Histoire

5. Mémoires, autobiographie

6. Biographie

7. Sciences sociales

8. Autres

9. Actualité

10 Médecine, guides

\begin{tabular}{|c|r|}
\hline \multicolumn{2}{|c|}{ Télévision } \\
\hline 1 & 1,5 \\
\hline 2 & 0,2 \\
\hline 3 & 28,3 \\
\hline 4 & 9,2 \\
\hline 5 & 16,4 \\
\hline 6 & 11,2 \\
\hline 7 & 7,8 \\
\hline 8 & 14,1 \\
\hline 9 & 4,5 \\
\hline 10 & 7,0 \\
\hline & (Effectif : 445 ) \\
\hline
\end{tabular}

Tableau 6 